\title{
Effects of transitive stimulus generalization on within -sets generalization and between -sets generalization
}

\author{
Dana S. Siira \\ West Virginia University
}

Follow this and additional works at: https://researchrepository.wvu.edu/etd

\section{Recommended Citation}

Siira, Dana S., "Effects of transitive stimulus generalization on within -sets generalization and between -sets generalization" (2005). Graduate Theses, Dissertations, and Problem Reports. 4192.

https://researchrepository.wvu.edu/etd/4192

This Dissertation is protected by copyright and/or related rights. It has been brought to you by the The Research Repository @ WVU with permission from the rights-holder(s). You are free to use this Dissertation in any way that is permitted by the copyright and related rights legislation that applies to your use. For other uses you must obtain permission from the rights-holder(s) directly, unless additional rights are indicated by a Creative Commons license in the record and/ or on the work itself. This Dissertation has been accepted for inclusion in WVU Graduate Theses, Dissertations, and Problem Reports collection by an authorized administrator of The Research Repository @ WVU.

For more information, please contact researchrepository@mail.wvu.edu. 
Effects of Transitive Stimulus Generalization on Within-Sets Generalization and Between-Sets Generalization

Dana S. Siira

A dissertation submitted in partial fulfillment of the requirements for the degree of Doctor of Education

in

Educational Psychology

\title{
College of Human Resources and Education at West Virginia University
}

\author{
Committee \\ Richard T. Walls, Chair \\ Daniel E. Hursh \\ Anne H. Nardi \\ Deborah J. Hendricks \\ Neal Shambaugh
}

Key Words: Generalization, Match-to-Sample, Generalization Training

Department of Advanced Educational Studies

Morgantown, West Virginia

2005 


\section{Abstract \\ Effects of Transitive Stimulus Generalization on Within-Sets Generalization and Between-Sets Generalization}

Dana S. Siira

The purpose of the study was to examine whether or not within-sets generalization (WSG) and between-sets generalization (BSG) are dependent on transitive stimulus generalization (TSG). A match-to-sample training procedure was used to train four typically developing five-year-old children to select stimuli from set A (fractions in ratio form) in the presence of stimuli from either set B or set $\mathrm{C}$ (fractions in picture form). The emergence of TSG, WSG, and BSG then was examined through presentations of stimuli involved in training and stimuli not involved in training.

Participants then were trained on the certain combinations of stimuli that were used to check for demonstrations of TSG. Demonstrations of TSG, WSG, and BSG then were examined after the TSG training in order to determine whether TSG training affected demonstrations of WSG or BSG. Participants then were trained on the certain combinations of stimuli that were used to check for demonstrations of WSG. Demonstrations of TSG, WSG, and BSG then were examined after the WSG training in order to determine whether WSG training affected demonstrations of BSG.

The data patterns of three of the four participants suggested that increased TSG training affected an increase in WSG responding, and the data pattern of only one of the four participants offered limited support that increased TSG training affected an increase in BSG responding. The data pattern of one of the four participants suggested that the addition of WSG training to TSG training affected an increase in BSG responding. 


\section{Dedication}

To my husband, James M. Siira, for everything we have and have to look forward to.

And

To my parents, Dr. David C. Stapleford and Dr. Elizabeth B. Stapleford, who shared with me their profound respect for the both the science and art of teaching. 


\section{Acknowledgements}

Sincere thanks are expressed to the members of my committee for their support throughout the construction of this document. Special thanks are expressed to Dr.

Richard T. Walls and Dr. Daniel E. Hursh for the enormous impact these two remarkable individuals had on my academic growth. 
Table of Contents

Chapter 1-Level of Instructional Analysis and Components of Instruction

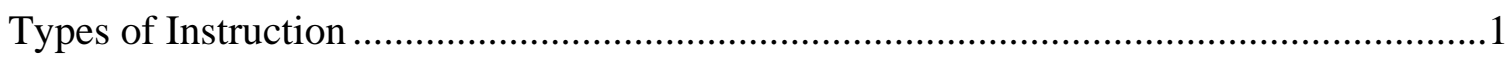

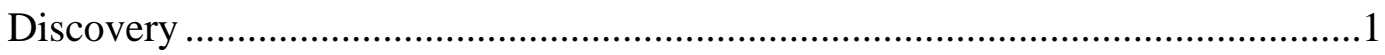

Discovery and repertoire changes ...................................................1

Discovery and success of instruction ................................................

Discovery and incorrect responses...................................................2

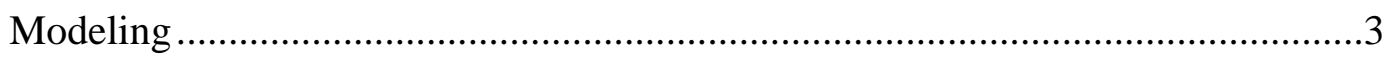

Modeling and repertoire changes.......................................................

Modeling and success of instruction..................................................

Modeling and incorrect responses ......................................................4

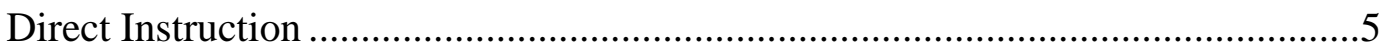

Direct instruction and incorrect responses ............................................5

Direct instruction and repertoire changes ..............................................5

Direct instruction and success of instruction .......................................6

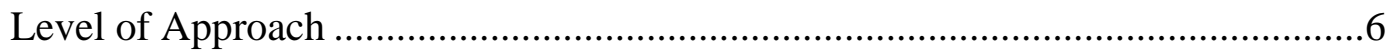

Goal and purpose ......................................................................6

Perspective on learning .................................................................

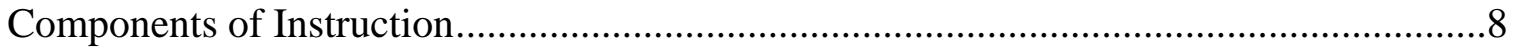

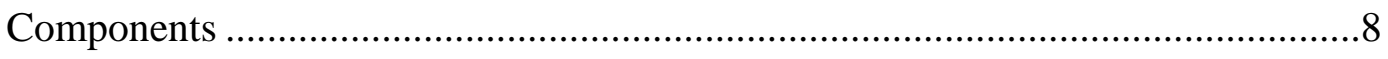

Sameness of stimuli ...................................................................8 
Range of variation among stimuli............................................................. 10

Positive and negative examples ....................................................11

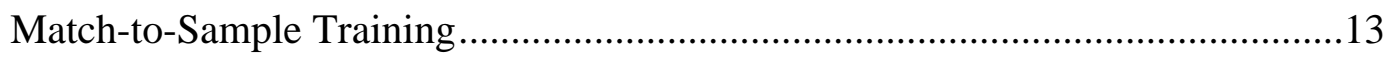

Conditional discriminations ........................................................13

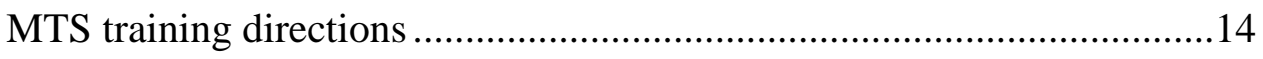

Chapter 2 - Types of Generalization and Review of Generalization Literature

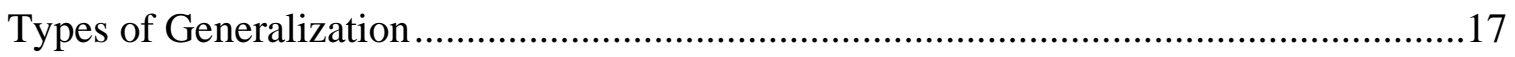

Transitive Stimulus Generalization..............................................................

Uses of Transitive Stimulus Generalization ....................................................18

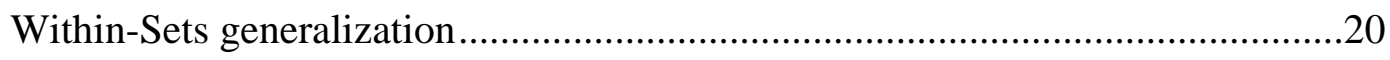

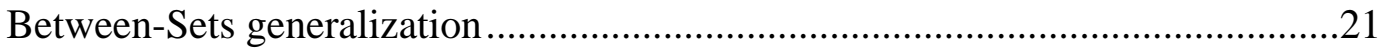

Uses of Within-Sets and Between-Sets Generalization ....................................22

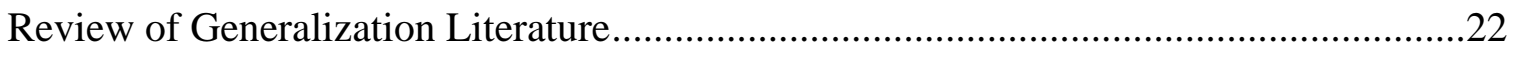

Response Extension Failure .................................................................22

Programming Generalization ...............................................................24

Training Across Multiple Sets ................................................................26

Effects of Generalization on Generalization..................................................28

Effects of within-sets and between-sets generalization on transitive stimulus generalization ....................................................28

Transitive stimulus generalization effect on extensions of responding .....29 
Possible transitive stimulus generalization effects on within-sets and

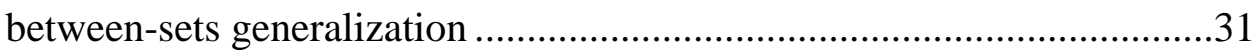

Match-to-Sample Training Effect on Transitive Stimulus Generalization............32

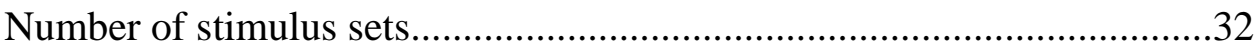

Simple discriminations ..................................................................33

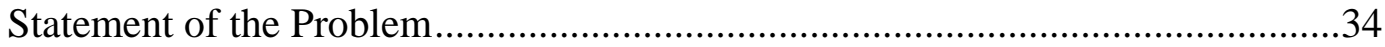

\section{Chapter 3 - Method}

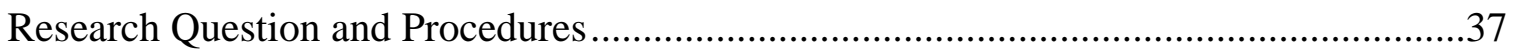

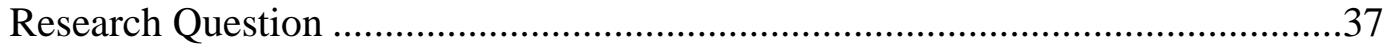

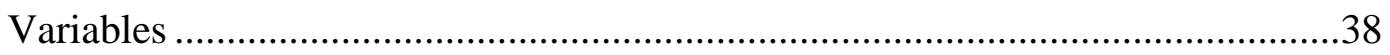

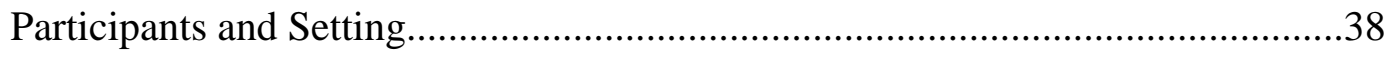

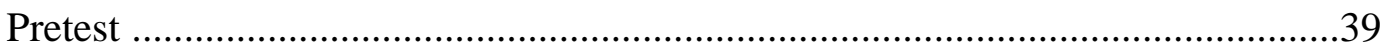

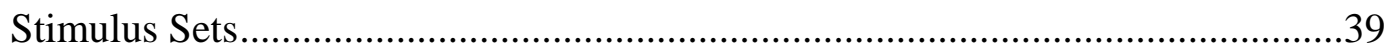

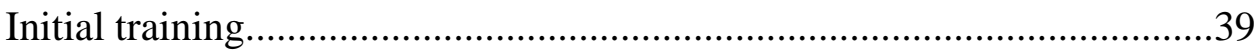

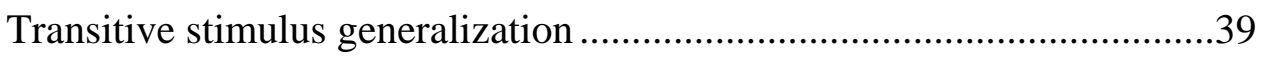

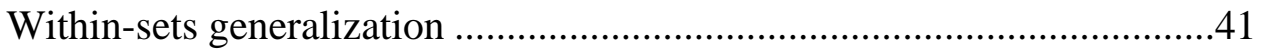

Between-sets generalization.........................................................4 41

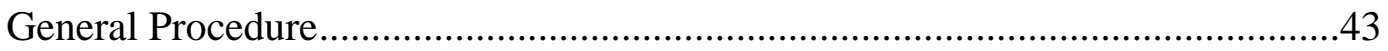

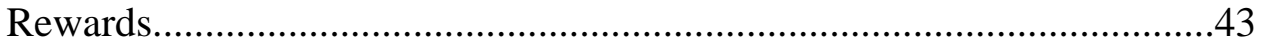

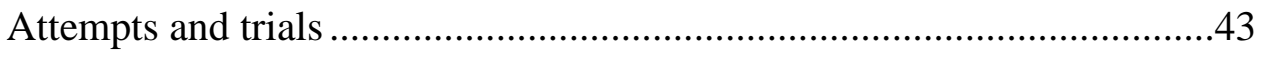

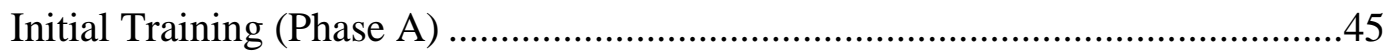




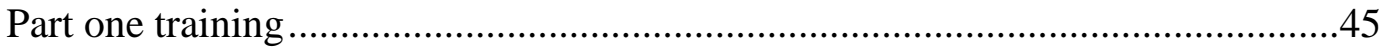

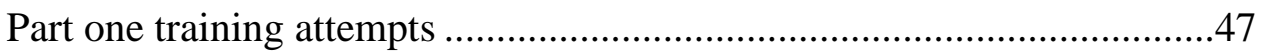

Part one training condition criteria .................................................48

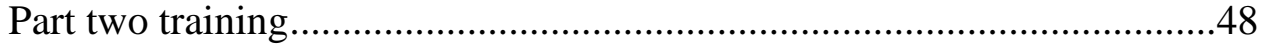

Part two training attempts ................................................................49

Part two training condition criteria .....................................................49

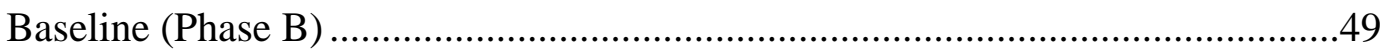

Baseline blocks .........................................................................50

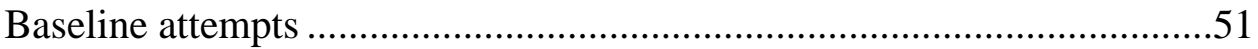

Initial training attempts in baseline ................................................51

Transitive Stimulus Generalization Training (Phase C) ......................................52

Phase C blocks ................................................................................52

Application of transitive stimulus generalization training........................53

Initial training attempts in Phase C .....................................................53

Within-Sets Generalization Training (Phase D) ................................................53

Phase D blocks ...............................................................................54

Application of within-sets generalization training................................54

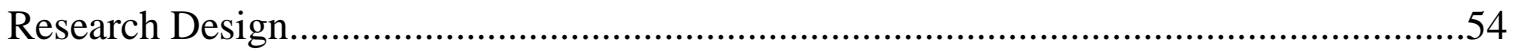

Summary of Consequences...........................................................................55

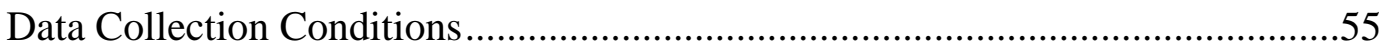

Repeating data collection conditions ................................................55

Phase C stability criteria ..............................................................5

Phase D stability criteria ..............................................................57 


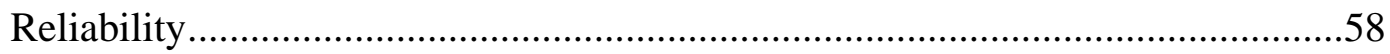

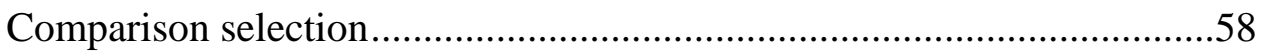

Delivery of the positive consequence .......................................................58

Chapter 4 - Results

Review of Research Questions and Data Collection Tables.................................................60

Review of Data Collection Conditions .........................................................................61

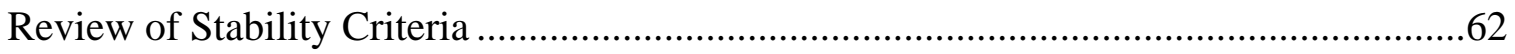

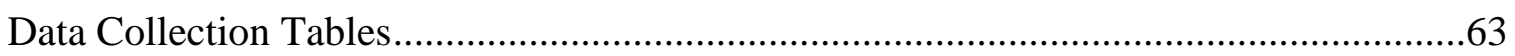

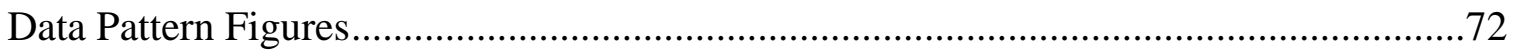

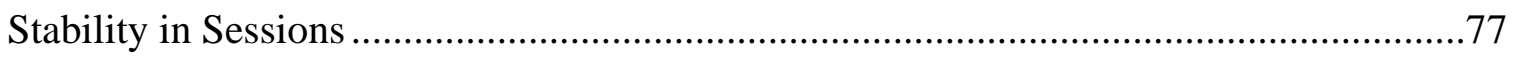

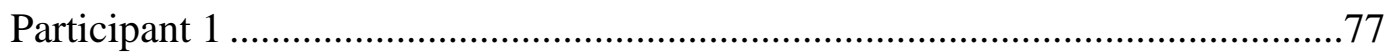

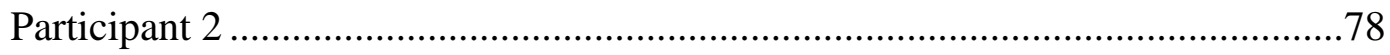

Participant 3 ...........................................................................................

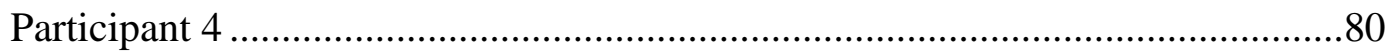

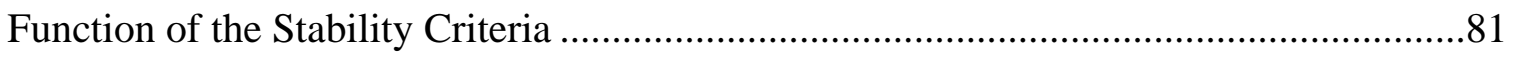

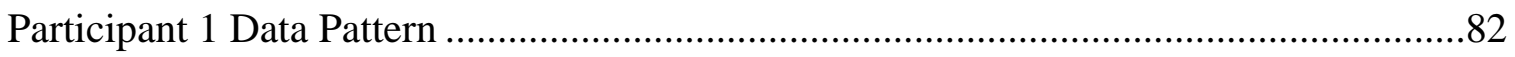

Participant 1 WSG responding .........................................................................82

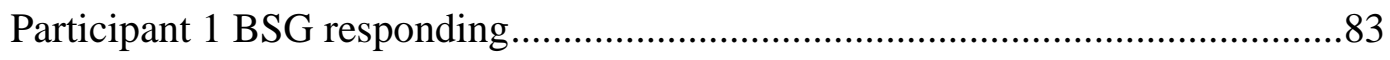

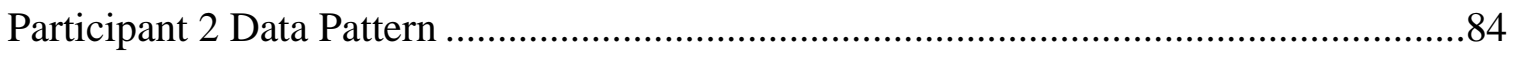

Participant 2 WSG responding ............................................................................

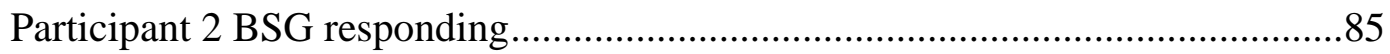




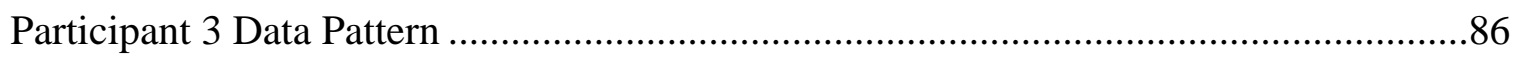

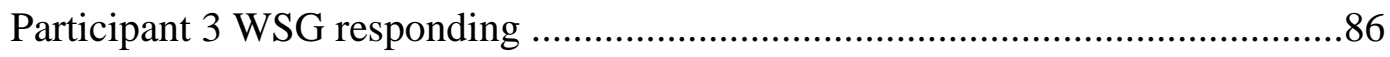

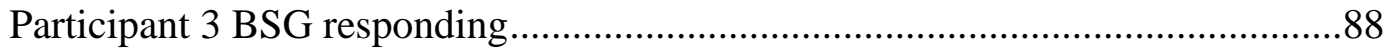

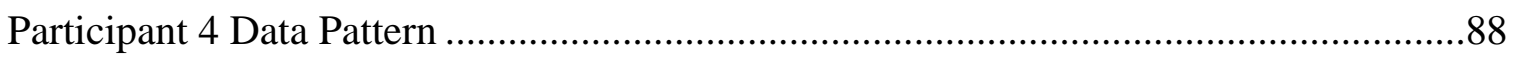

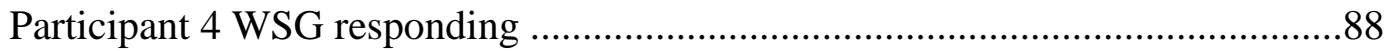

Participant 4 BSG responding..............................................................90

Summary of Results in Terms of the Research Questions...........................................91

Chapter 5 - Discussion

The First Research Question Result...................................................................93

The Second Research Question Result ....................................................................95

Relations Among Types of Generalization..........................................................97

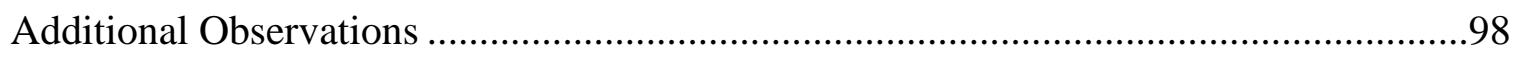

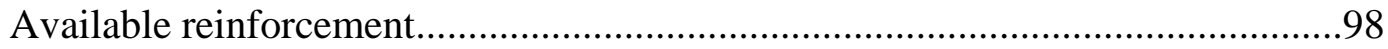

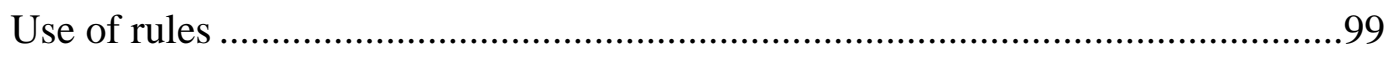

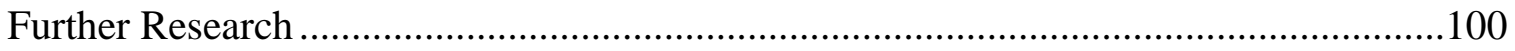

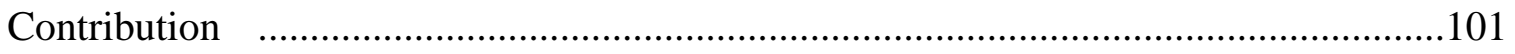

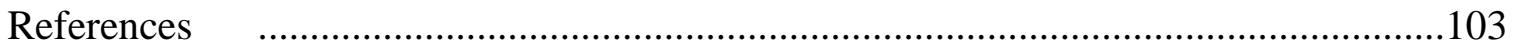

Appendices

Appendix A- Sample Part One and Part Two Initial Training Scorecards ..........108

Appendix B- Sample Ordering of Attempt Cards in Two Baseline Blocks ........110 
Appendix C- Sample Baseline Block Scorecard .....................................................111 


\section{List of Tables}

Table 1: Correct Selections Made by Participant 1 in Each Phase, by Type of Attempt

Card (TSG, WSG. BSG, and IT) and Session ..............................................64

Table 2: Correct Selections Made by Participant 2 in Each Phase, by Type of Attempt

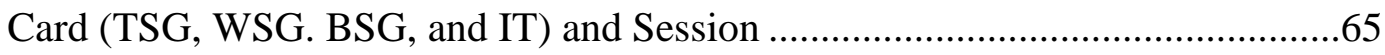

Table 3: Correct Selections Made by Participant 3 in Each Phase, by Type of Attempt

Card (TSG, WSG. BSG, and IT) and Session

Table 4: Correct Selections Made by Participant 4 in Each Phase, by Type of Attempt

Card (TSG, WSG. BSG, and IT) and Session 


\section{List of Figures}

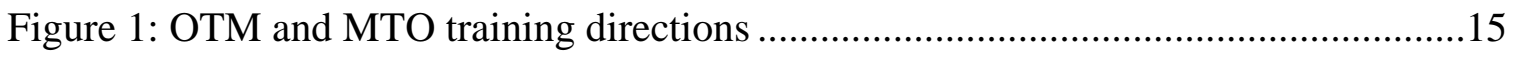

Figure 2: Stimulus sets used in initial training and transitive stimulus generalization

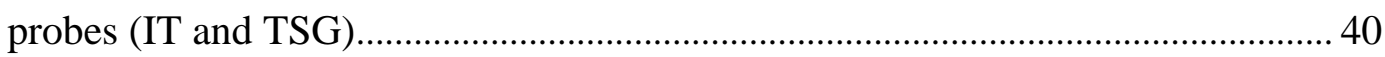

Figure 3: Stimulus sets used in within-sets generalization (WSG) probes......................42

Figure 4: Stimulus sets used in between-sets generalization (BSG) probes....................44

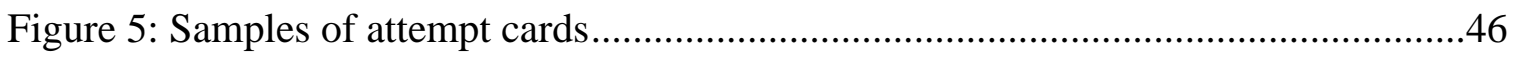

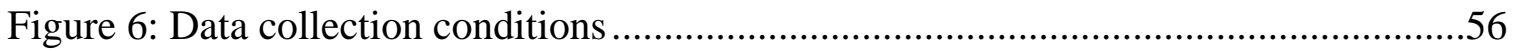

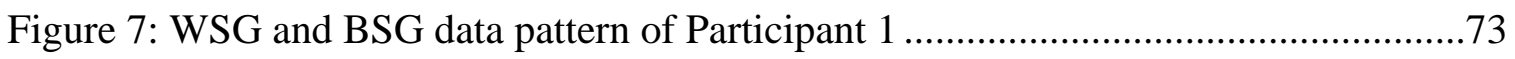

Figure 8: WSG and BSG data pattern of Participant 2 ...............................................74

Figure 9: WSG and BSG data pattern of Participant 3 .............................................75

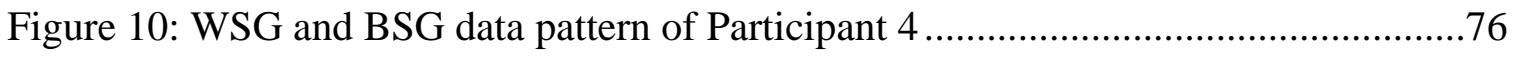


Chapter 1: Level of Instructional Analysis and Components of Instruction Types of Instruction

All instruction involves an instructor (person or machine) that manipulates some aspect of the student's environment in order to change the student's repertoire. In that sense, all instruction may be considered to fall somewhere on a continuum of teacher involvement in the arrangement of instructional stimuli.

The extent to which the instructor manipulates stimuli and the presentation of those stimuli that eventually are to gain control over a response (i.e., become associated with the availability of reinforcement following a certain response) may vary based on where the instruction falls on the continuum. In the first part of this paper, three types of instruction (Discovery, Modeling, and Direct Instruction) that fall on different places on the continuum will be discussed in terms of (1) how repertoire changes (i.e., learning) are accounted for, (2) what is considered necessary for instruction to be successful, and (3) what teachers can do to facilitate repertoire changes when an incorrect response is given. Discovery

Discovery and repertoire changes. Discovery learning relies on students working through teacher-arranged activities. Repertoire changes are said to occur during Discovery instruction when students contact new relations among stimuli in the course of working through the activities. Activities are arranged so that students contact, or discover, general case phenomena as a result of working through the instructional activities (Bruner, 1961).

Discovery and success of instruction. Paramount to the success of Discovery as a form of instruction is a student's base knowledge, meaning that a sufficient repertoire of 
background knowledge is necessary (Bruner, 1960). Prior to Discovery, background information must be structured in such a way so that "not only a specific thing but a model for understanding other things like it that one may encounter” are part of the repertoire (Bruner, 1960, p. 25). That is, the availability of problem solving heuristics is a necessary component of Discovery learning.

Once a student has a sufficient repertoire to benefit from Discovery instruction, the success of the instruction then relies on that student using prior knowledge to work through instructional activities that encourage students to contact (i.e., discover) new relations or associations among what they learned previously. Prior knowledge used in Discovery activities may be the result of previous Discovery activities or some other form of instruction.

Discovery and incorrect responses. Incorrect responses are to be expected as a student works through Discovery instructional activities. Students try different avenues in overcoming problems as part of a Discovery process. A situation in which the student cannot work through an activity by trying different paths, however, is explained as the result of an inadequate repertoire. In this case the teacher may arrange other activities for the purpose of facilitating the discovery of necessary background knowledge, or use a different form of instruction that may be more efficient for the particular information that is presumed to be missing from the repertoire.

In a case where a student cannot work through an activity, the exact manner in which Discovery activities should be altered may not be clear. One reason for this is that Discovery activities may not enable the instructor to identify precisely what information is missing from the repertoire. Discovery activities that are closely monitored may be 
helpful in pointing out to the teacher additional instructional examples and non-examples that could be useful in affecting student performance; however, the exact responses that are not part of the student's repertoire may not be affected efficiently through further instruction presented through Discovery activities.

Discovery learning, in and of itself, is a molar-level approach to instruction in that the teacher cannot affect specific student responses as they occur. Instruction at the molar level is appropriate for students who have sufficient repertoires, but when students have insufficient repertoires, molar-level instruction may act as a barrier for predictable instructional outcomes.

\section{Modeling}

Modeling and repertoire changes. Modeling relies on students observing the behaviors of a model (i.e., the instructor). Repertoire changes (i.e., learning) are said to occur when new behaviors are demonstrated by the student that were not part of the student's repertoire prior to being exposed to the model's behavior (Bandura, 1969). Modeling and success of instruction. The pivotal component leading to the success of instruction presented through modeling is found in the student's behavior. Bandura (1969) stated that a student must attend to the modeling performance if learning is to occur. The teacher (model) may encourage student attention through altering features such as size, color, and shape of instructional stimuli, which may make students more likely to watch modeled performances. Reinforcing effects that maintain watching modeled performances may weaken, however, leaving the instructional situation without its most salient component -- the student's attention. 
In the perspective taken in this paper, that loss of student attention does not point out a flaw in Modeling as an approach to instruction, but rather points out that a description of the instructional situation at a molar level (as opposed to a molecular level) may not lend itself as well to describing what an instructor could do to affect a specific change in the student's behavior.

Modeling and incorrect responses. When a response is given by a student after attending to a modeled demonstration, the teacher may provide feedback to the student on whether or not his or her response was correct. Consequences of the student's response in the form of the teacher letting the student know the response was either correct or incorrect are not expected to have reinforcing effects on certain responses unless the student is aware of the reinforcing contingency (Bandura, 1977). That is, if the student is not aware under what circumstances (i.e., what instructional stimuli must be present) a particular response is to be followed by a positive consequence, the potentially reinforcing consequence will not have a reinforcing effect on the response.

Bandura (1977) described the student's awareness of reinforcing contingencies as intermediary cognitive responses between external influences on the response (e.g., the instructional stimuli and the teacher's feedback) and the student's overt response. Thus, if a student produces an incorrect response, the teacher must model another performance for the purpose of helping the student become aware precisely of what aspect of the situation made the response incorrect. A question remains, however, pertaining to what contingencies are available to alter the teacher's behavior to indicate what components of his or her modeling performance should be altered in order to facilitate correct responses from the student. 


\section{Direct Instruction}

Direct instruction and incorrect responses. One way the teacher may contact contingencies that are reinforcing to his or her modeling behavior (e.g., correct responses from the student) is to slowly alter instructional stimuli that the student responds to correctly until the student no longer responds correctly. That practice may allow the teacher to contact a situation in which a small and specific change in instructional stimuli affects the difference between a correct and incorrect response.

A teacher's arrangement of instructional stimuli as was described in the previous paragraph is recommended by Direct instruction as a way the teacher may affect incorrect student responses to instructional stimuli. Direct instruction focuses on training students to respond to relations among subsets of instructional stimuli so that they may respond to the entire set without further training (Engelmann, Becker, Carnine, \& Gersten, 1988).

Direct instruction and repertoire changes. A Direct approach to instruction enables a teacher to examine repertoire changes at a more molecular level compared to a strictly Discovery or Modeling approach. Direct instruction involves the presentation of instructional stimuli that vary only slightly from stimuli the student previously has responded to correctly. If a student does not respond to instructional stimuli that vary in

just a few aspects from stimuli he or she has responded to, the teacher is more apt to identify the aspects of the stimuli to which the student cannot respond (Engelmann \& Carnine, 1982).

Once components of the stimuli that the student does not respond correctly to are identified, the teacher can correct the student in the presence of the new stimuli. A repertoire change then is observed when the student predictably demonstrates the correct 
response in presence of the stimuli. That is, as other stimuli are altered (e.g., the teacher stops supplying additional prompts) the student still responds correctly in presence of the instructional stimuli. In that case, the instructional stimuli may be called a discriminative stimulus which is associated with the availability of reinforcement (e.g., praise or perhaps escape from demands) following a certain response (i.e., the correct answer) (Skinner, 1953). Thus, repertoire changes are observed when a student demonstrates a certain response in the presence of stimuli in which he or she previously did not demonstrate the response.

Direct instruction and success of instruction. Direct instruction describes several components of instructional stimuli that are necessary for predictable success of instruction. Those aspects, described by Engelmann and Carnine (1982), are discussed in the second part of this paper.

\section{Level of Approach}

As was stated earlier, all instruction may be considered to fall somewhere on a continuum of teacher involvement in the arrangement of instructional stimuli. Modeling, Discovery, and Direct instruction are three types of instruction that all involve an instructor who arranges instructional stimuli, and could be best described as holding different places along the continuum. For example, Direct instruction and Discovery could be described as falling toward opposite ends of the continuum with Modeling falling somewhere in the middle.

Goal and purpose. The goal of this paper is not to encourage the use of one approach to instruction over another. Each type of instruction described in the preceding paragraphs has a place as a valuable tool for altering student repertoires, and the use of 
one of those tools (i.e., one type of instruction) does not imply that the use of additional tools is inappropriate.

The purpose of this paper is to discuss the value of instruction that enables the instructor to affect the instructional situation at a molecular level. A molecular-level examination of instruction, in this paper, refers to an explanation of responding based on contact with a learning process that is controllable by the instructor.

Perspective on learning. The view taken in the current paper is that learning (or a change in repertoire) is simply a change in behavior. Although such behavior changes involved in learning are often covert, they are still behaviors which are subject to principles of behavior change (Skinner, 1957).

Thus, it is the stance of this paper that in order to affect the most predictable changes in student responding, instruction must be considered at a molecular level as opposed to a more molar level. That is, an instructor must deliberately manipulate (a) stimuli and (b) the presentation of those stimuli such that even small changes in the stimuli affect predictable changes in responding. In that way, an instructor may more easily identify aspects of stimuli that have been associated with incorrect responses and alter those stimuli until a correct response occurs. Regardless of the type of instruction used, if the instruction fails in some way (i.e., the student does not respond), an instructor who utilizes a molecular analysis of the situation may contact contingencies that enable him or her to affect student behavior in a more deliberate fashion.

In the next part of this paper, components of effective instruction that were described by Engelmann and Carnine (1982) will be discussed. The purpose of the following section is to highlight components of instruction that a teacher may directly 
affect, and hence, are components that may be altered to produce specific changes in a student's responding.

\section{Components of Instruction}

Necessary components of instruction have been described concisely by Engelmann and Carnine (1982) and will be used as a framework for the following molecular-level discussion of principles of instruction for affecting predictable changes in student behavior. A discussion then follows on how the application of those principles leads to generalization (i.e., an extension of responding based on past experience).

Components for effective instruction described by Engelmann and Carnine (1982) have been supported empirically (e.g., Tennyson 1973; Werts, 1991; White, 1988). Specific programs developed by Engelmann and his colleagues, called Direct Instruction, have been shown to affect strong learning outcomes for students with a wide range of repertoires (Adams \& Engelmann, 1996; White, 1988). Such outcomes suggest that molecular-level approaches to instruction, such as Direction Instruction, are likely to yield predictable positive learning outcomes for various groups of students. The following paragraphs are a molecular-level discussion of components of instruction, as outlined by Engelmann and Carnine (1982).

\section{Components}

Sameness of stimuli. Engelmann and Carnine’s (1982) principles for effective instruction hold that a sharp distinction does not exist between the establishment of stimulus control over correct responding (i.e., initial learning) and the extension of responding based on past experience with related stimuli (i.e., generalization). That is because generalization is considered to be a function of the sameness among (1) a subset 
of stimuli used to gain control over correct responses and (2) the rest of the stimuli in the set, in the presence of which, the correct responses also occur.

All stimuli within the training subset must share a certain sameness if the student is to respond in a predictable way to all stimuli in the subset. It is possible then that after some training on stimuli in a subset, correct responses to other stimuli in the subset could occur before direct training. Therefore, the point at which responding may be most accurately called initial learning rather than generalization (i.e., an extension of responding based on past experience with related stimuli) is not clear. Thus, demonstrations of generalization may be called demonstrations of student learning (Engelmann \& Carnine, 1982).

The preceding description of generalization (or demonstrations of student learning) as a function of sameness among instructional stimuli points out an aspect of the instructional situation that could be altered in the case of a student's incorrect response. If a student responds incorrectly to instructional stimuli, the instructor may examine the situation along the lines of how similar the current stimuli are to past stimuli to which the student did respond correctly. Once a difference in similarity is identified, the instructor may supply a prompt (perhaps supply the correct response or an approximation of the correct response) so that the student can give the correct response. In that case, the student's correct response then may be reinforced in the presence of the instructional stimuli.

In the situation that was just described, there is no reason to assume that reinforcement must be supplied by the teacher. For instance, reinforcement could be supplied by the student identifying (possibly due to the similarity of the current response 
to past responses) that the correct response was given. That is, a covert behavior could serve as reinforcement for the response (Skinner, 1957).

As described before, generalization is a function of sameness among instructional stimuli. The following section describes increasing the range of variation among stimuli as a way of increasing the sameness among (a) stimuli of a subset presented as part of instruction and (b) other stimuli of the set. More specifically, the following section describes increasing the range of variation among stimuli to include all components that are considered to be salient qualities of stimuli, in the presence of which, the response is to occur (i.e., generalize).

Range of variation among stimuli. In order for generalization demonstrations to be successful (i.e., correct responses on generalization tests), certain responses must occur in the presence of stimuli that share a certain sameness with stimuli, in the presence of which, the response was initially reinforced. For example, suppose training were to occur on a subset of stimuli that consisted of circles and squares of two different sizes that were either red or blue, and training involved students responding “yes” to red circles, "no” to blue circles, “yes” to blue squares, and "no” to red squares. Generalization, as an extension of responding based on past experience with related stimuli, may involve the student responding “yes” to red circles of a size not seen in training and "no" to blue circles of sizes also not seen in training. Correct responding on such generalization tests would be explained by the sameness in color and shape that previously had been associated with a certain response (i.e., the correct answer) (Engelmann \& Carnine, 1982; Skinner 1957). 
In the example discussed in the previous paragraph, red circles of sizes not included in the training subset shared a sameness of color and shape that previously had been associated with a certain response. That is, the stimuli not included in training shared a sameness of quality with the stimuli from the subset used to gain control over correct responses during training (Engelmann \& Carnine, 1982). Had the training subset not included shapes of various sizes, the student may have been trained unintentionally to respond correctly only to red circles or blue squares that were of specific sizes. Thus, the range of variation among stimuli that are considered to be representative of the salient qualities (e.g., color, shape, and size) should be included within the training subset (Engelmann \& Carnine, 1982; Silbert, Carnine, \& Stein, 1981).

The next section describes positive examples in instruction as stimuli that are representative of the salient features of a stimulus set. Negative examples are stimuli that do not represent enough of the salient features of a set to be associated with the response that occurs in the presence of positive examples. The presence of positive and negative examples are discussed as necessary in instruction because they may indicate the limits on the range of stimuli that reinforcement for a certain response will follow.

Positive and negative examples. In the previous sections, correct responses in the presence of stimuli similar to the training stimuli (i.e., generalization) were described as functions of the sameness among training stimuli and stimuli used to test generalization. Engelmann and Carnine (1982) described a mechanism that "classifies new examples as either positive examples of the concept or negative examples” (Engelmann \& Carnine, 1982, p. 4). 
Reference to a mechanism that guides covert responses (e.g., thoughts) and overt responses (e.g., selecting a correct response) to stimuli may be avoided by describing a positive example as a collection of stimuli that a certain response (i.e., the correct answer) has been reinforced in the presence of, such that the presentation of the positive example signals the availability of reinforcement contingent on a certain response (i.e., the correct answer). It then follows that if certain conditions are present that temporarily decrease the effectiveness of the reinforcer associated with the positive example (e.g., satiation with praise for correct responses), the correct response will not follow the presentation of the positive example (Keller \& Schoenfeld, 1950; Michael 1982; Skinner, 1953).

Regardless of the potential reinforcing effectiveness of consequences that are contingent on correct responses, correct responses should not be expected to follow examples that do not contain enough of the stimuli that, in the presence of which, correct answers were reinforced. Engelmann and Carnine (1982) described such examples as negative examples.

Negative examples (i.e., stimuli that do not contain enough of the properties of stimuli that are called positive examples) may be useful in describing the range of stimuli that reinforcement for a certain answer will follow. For example, Tennyson (1973) found that students who were given what were called negative instances in addition to positive instances of adverbs in sentences (the concept being taught) showed better acquisition of the concept, presumably because they were exposed to a range of stimuli that included the limits of positive examples. 
In the example of training students to respond in a certain way to certain shapes of a certain color discussed in the previous paragraphs, blue circles were examples of stimuli to which "yes" was not a correct answer. Thus, the presentation of a blue circle would be a negative example of stimuli to which the response of "yes" was correct. Without the presence of those negative examples, a student may respond "yes" to circles of any color.

In the previous paragraphs, (a) sameness of stimuli, (b) range of variation among stimuli, and (c) positive and negative examples were used to described aspects of instruction that could be manipulated in order to prevent the occurrence of incorrect responses. After a set of instructional stimuli is arranged based on the components of instruction described previously, a method for presenting the instructional stimuli may be considered. Based on the student's repertoire, certain presentation styles (e.g., Modeling, Discovery activities, or Direct instruction) may have more or less utility.

The focus of this paper now switches from a discussion of components of instruction to different types of generalization (i.e., learning) that instruction affects. Match-to-sample training is described below as a direct method for teaching relations among stimuli. A description of match-to-sample training follows because it is an instructional technology that lends itself well to examinations of instruction at a molecular level such that types of generalization may be examined in terms of the type of relations among stimuli that are demonstrated.

\section{Match-to-Sample Training}

Conditional discriminations. Match-to-sample (MTS) training is a direct approach to teaching relations among different sets of stimuli that are referred to as 
sample and comparison stimulus sets. Training involves reinforcing the selection of a stimulus from a comparison set in the presence of a stimulus from a sample set. For example, if a sample set contained three shapes (e.g., a triangle, a circle, and a square) and a comparison set contained three numbers (e.g., one, two, and three) MTS training would involve reinforcing the selection of a comparison stimulus, such as two, in the presence of a sample stimulus, such as circle. After MTS training, if the sample stimulus circle were shown to control selections of the comparison stimulus two (i.e., the stimulus two was predictably selected in the presence of the stimulus circle), the selection of two in the presence of circle would be referred to as a conditional discrimination.

MTS training directions. Match-to-sample procedures used to train the selection of a certain stimulus from a comparison set of stimuli conditionally on the presentation of a certain stimulus from a sample set of stimuli can lead to positive outcomes on tests of untrained conditional discriminations among those stimulus sets. Many different matchto-sample procedures have been used successfully throughout the literature. Two types of match-to-sample training, called match-to-sample training directions, are described below.

Figure 1 is an illustration of two different match-to-sample training directions for three sets of stimuli (Set A, Set B, and Set C). The one-to-many (OTM) training direction involves training participants to select a comparison stimulus from either the entire Set B or Set C conditionally on the presentation of a sample stimulus from Set A. In other words, three $\mathrm{AB}$ conditional discriminations (A1B1, A2B2, and A3B3) and three AC conditional discriminations (A1C1, A2C2, and A3C3) would be trained. The many-toone (MTO) training direction involves training participants to select a comparison 

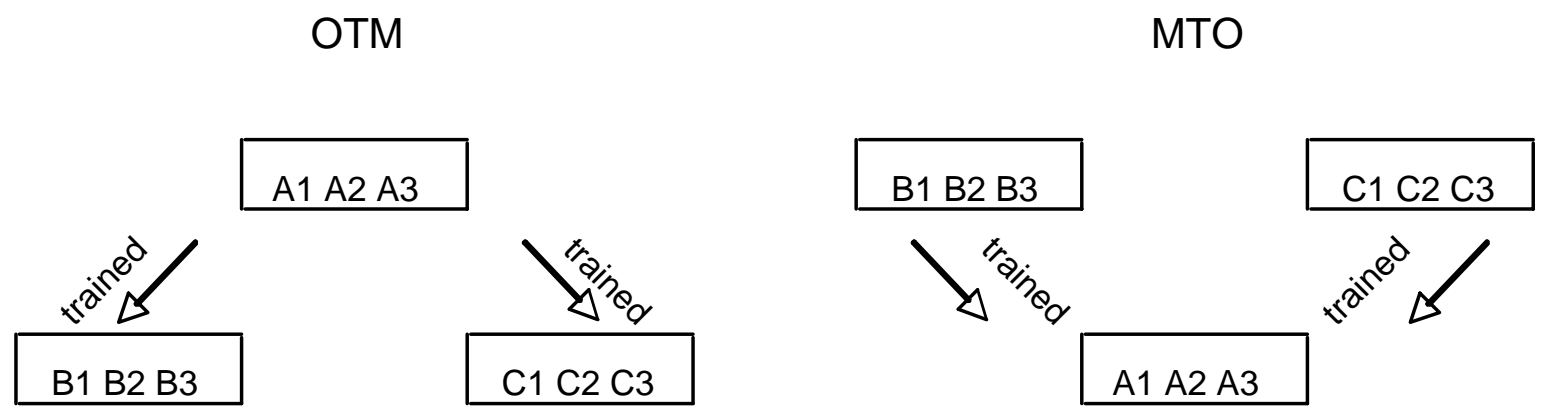

Figure 1. OTM and MTO training directions. 
stimulus from the entire Set A conditionally on the presentation of a sample stimulus from either the Set B or Set C. Thus, three BA conditional discriminations (B1A1, B2A2, and B3A3) and three CA conditional discriminations (C1A1, C2A2, and C3A3) would be trained.

Different types of generalization describe different types of repertoire change. Three different types of generalization are discussed in the next section of this paper in terms of the type of relations among stimuli that are demonstrated. The purpose of the next section is to provide a description of different types of generalization (i.e., learning) in terms of relations among stimuli that are trained as a result of instruction. In the next section, match-to-sample training is discussed in the descriptions of the types of generalization. That is because match-to-sample is an instructional technology that lends itself well to examinations of instruction at a molecular level such that types of generalization may be examined in terms of the types of relations among stimuli that are demonstrated. 
Chapter 2: Types of Generalization and Review of Generalization Literature Types of Generalization

\section{Transitive Stimulus Generalization}

Stimuli within the training subsets must share a certain sameness if the student is to respond in a predictable way to all stimuli in the subsets. The sameness among stimuli used in MTS training may be limited to only the stimuli used in training. Consider the sample set A (triangle, circle, and square), the comparison Set B (one, two, and three), and the comparison Set C (X, Y, and Z). After MTS training, sameness among stimuli in Set A may be described by the similar control each stimulus in Set A has over the selection of a certain stimulus in Set B and a certain stimulus in Set C. That is, corresponding stimuli in Set $\mathrm{A}$, Set $\mathrm{B}$, and Set $\mathrm{C}$ share a sameness in that they make up conditional discriminations as a result of the MTS training. After training for the conditional discrimination 'triangle' and 'one' and the conditional discrimination 'triangle' and ' $\mathrm{X}$ ', generalization (i.e., an extension of responding based on past experience) would be shown if either 'one' controlled selections of ' $X$ ' or ' $X$ ' controlled selections 'one.' That is, generalization would be shown if either the conditional discrimination 'one' and ' $\mathrm{X}$ ' or the conditional discrimination ' $\mathrm{X}$ ' and 'one' were shown. Haring, Breen, and Laitinen (1989) referred to that type of generalization as transitive stimulus generalization.

A demonstration of 'one' and ' $\mathrm{X}$ ' or a demonstration of ' $\mathrm{X}$ ' and 'one' is called transitive stimulus generalization because it is a demonstration of a transitive relation. Transitivity refers to sample and comparison stimuli that are related through a demonstrable relation with a common stimulus. That is, if the (triangle, one) and 
(triangle, X) discriminations were demonstrable, a demonstration of the (one, X) discrimination would demonstrate transitivity. In that case, the sample and comparison stimuli would be related through a demonstrable relation with the common stimulus 'triangle.'

In more general terms, if $\mathrm{BA}$ and $\mathrm{AC}$ discriminations were demonstrable, a demonstration of $\mathrm{BC}$ or $\mathrm{CB}$ discriminations would demonstrate transitivity. In that case, the sample and comparison stimuli would be related through a demonstrable relation with the common stimulus A (Sidman \& Tailby, 1982). Thus, a demonstration of BC or CB conditional discriminations are referred to as demonstrations of transitive stimulus generalization because sample and comparison stimuli involved in BC or CB conditional discriminations are related through a demonstrable relation with a common stimulus.

Training that facilitates transitive stimulus generalization is an efficient instruction tool in that the training has a generative quality. That is, instruction on a small number of relations among stimuli occasions the demonstration of several addition relations among stimuli that were not included in training. For example, a trained relation between (1) stimulus B and stimulus A and (2) stimulus C and stimulus A can occasion the demonstration of untrained relations through the intermediary stimulus A. In this example, those untrained relations include (1) stimulus C and stimulus B and (2) stimulus B and stimulus C.

Uses of Transitive Stimulus Generalization

If instruction (e.g., MTS training) lends itself to checks on the demonstrability of transitive stimulus generalization, checking for transitive stimulus generalization may provide information to the instructor on how successful the instruction was at facilitating 
responding to important relations among stimuli that were used as part of instruction. That is, transitive stimulus generalization may provide information with respect to how effective the instruction was. For instance, correct responses to $\mathrm{CB}$ and $\mathrm{BC}$ relations that were described in the previous paragraph indicate that the student responds to relations among stimuli in topographically different presentations from training.

Since transitive stimulus generalization describes relations among stimuli involved in training, the stimuli that are used in training sets may be arbitrary. That is, transitive stimulus generalization may still be shown if relations among stimuli used in training sets are limited exclusively to other stimuli used in training. For example, stimulus sets of nonsense symbols could be used in training and tests of transitive stimulus generalization because the transitive relation relies on a common intermediary stimulus involved in other trained relations. Transitive stimulus generalization does not evaluate whether or not training occasions correct responding to stimuli not used in training.

Stimulus relations evaluated in tests for transitive stimulus generalization have been used extensively in basic research (see Zentall, Galizio, \& Critchfield, 2002 for a review) as part of evaluations of stimulus relations that may be unique to human verbal behavior. In more applied settings, transitive stimulus generalization may be used to determine if training affected the student's repertoire such that relations not directly involved in training are demonstrable after training on certain other relations. Thus, transitive stimulus generalization may be used to describe training efficiency.

If the relations among stimuli that are trained have utility outside of the MTS training context, other types of generalization, such as within-sets or between-sets 
generalization, may be examined. Within-sets generalization and between-sets generalization are described in the next paragraphs as extensions of responding from trained instructional stimuli to other stimuli.

\section{Within-Sets Generalization}

The sameness among stimuli used in MTS training may not be limited to only the stimuli used in training in that sameness among stimuli could describe stimuli not used in training that are part of a larger set of stimuli. For example, medium sized red and blue circles share shape and color with stimuli in the comparison sets that were described in previous paragraphs. Medium sized red and blue circles from Set M may then be described as stimuli that are part of a larger set including small and large, red and blue circles. After MTS training on AB and AC conditional discriminations, generalization (i.e., an extension of responding based on past experience) would be shown if stimuli from Set A were shown to control correct selections of stimuli from Set M. Haring et al. (1989) referred to that type of generalization as within-sets generalization.

Within-sets generalization is described as an extension of responding from sets involved in training to sets containing stimuli that are part of a larger set that include the stimuli used in training, such that all stimuli in the larger set share a sameness of essential features (Harding et al., 1989). Thus, during MTS training for AB and AC conditional discriminations, demonstrations of AM discrimination would show within-sets generalization. 


\section{Between-Sets Generalization}

In addition to sameness among stimuli described by demonstrations of transitive stimulus generalization and within-sets generalization, sameness among stimuli may be described by between-sets generalization. Between-sets generalization is described as an extension of responding from sets that were involved in training to different sets that do not contain the stimuli from sets used in training (Haring et al., 1989). For example, after training on the set of small and large, red and blue circles described in previous paragraphs, between-sets generalization may be examined on a new set of stimuli, such as a set of red and blue squares of various sizes called Set S. Between-sets generalization then would be demonstrated by stimuli from Set A (yes and no) controlling selections of from Set S.

Set S (red and blue squares of various sizes) is not part of the Set B (large red circles and large blue circles) or Set C (small red circles and small blue circles), in that Set S stimuli do not share all essential features of Set B and Set C, namely, a circular shape. In order for an extension of responding to occur, however, some sameness of stimuli must be present. That is, some stimulus involved in the generalization condition must serve as a discriminative stimulus that is associated with a response that is either itself the correct response or a response that serves as a discriminative stimulus for another response that is the correct response (see Sidman \& Tailby, 1982; Skinner, 1957). Thus, generalization of any kind involves some degree of sameness among stimuli present during training and stimuli present during a generalization condition (Engelmann \& Carnine, 1982). 


\section{Uses of Within-Sets and Between-Sets Generalization}

Following classroom instruction, within-sets and between-sets generalization (as opposed to transitive stimuli generalization) are likely to be the types of generalization that are sought because they represent extensions of responding from trained instructional stimuli to stimuli not involved in training. Thus, instructors who wish for repertoire changes that occur in the classroom to affect other responses in a variety of settings may be more interested in training that facilitates extensions of responding from trained stimuli to other stimuli.

In sum, generalization, when described as an extension of responding based on past experience with related stimuli, may be considered to be a function of the sameness among (1) stimuli used in training and (2) stimuli present in situations in which generalization is examined. As is discussed in the following review of generalization literature, contact with such sameness among stimuli may be arranged by an instructor through programming the type of generalization sought or through training across multiple sets of stimuli.

\section{Review of Generalization Literature}

\section{Response Extension Failure}

Garcia, Baer, and Firestone (1971), used shaping and fading procedures to train two adolescent participants, described as persons with severe mental retardation, to imitate vocal responses. Training was done on short vocal sounds, which were vowel sounds (e.g., "a” as in Pat). Vocal sounds similar to those used in training (i.e., other vowel sounds) were then presented to participants as probes for within-sets 
generalization. A demonstration of within-sets generalization was described if the participant imitated the vowel sound.

Long vocal sounds were words containing consonant sounds and a vowel sound used in the within-sets generalization probes (e.g., "it" or "pet”). A demonstration of between-sets generalization was described if the participant imitated a long vocal sound when it was presented. Garcia et al., (1971) found that for both participants, demonstrations of within-sets generalization increased with increase exposure to training, however, no demonstrations of between-sets generalization were seen. In other words, training did not occasion the extension of responding (i.e., demonstration of the imitating skill) in the presence of stimuli from untrained sets.

Parsonson and Baer (1978) examined generalization of improvisational skills in five typically developing preschool children across sets of tools. Improvisation skills training was a process in which the researcher (1) presented an exemplar tool from a set, such as a toy hammer, (2) explained what the child was to use it for (e.g., use this tool to hit that peg), and (3) gave descriptive feedback and praise for using the tool. Other tools in the set were items that, through improvising, could be used in place of the exemplar tool. For example, other members of the hammer set were items such as a brick, as well as items that could be used in combination such as a drilled block and a rod. Prior to the start of training, all participants could demonstrate how to use the exemplar tools.

After training on improvisation items from a set began, within-sets generalization was described when a participant used an item from a tool set (i.e., improvised) before he or she was trained how to use that particular item. Between-sets generalization was 
described when a participant used an item from a set of tools before training had been done on the set.

Results of Parsonson and Baer (1978) and Garcia et al. (1971) showed that within-sets generalization was dependent on training. Between-sets generalization, however, was not demonstrated. The authors suggested the limitation in generalization was a result of the focus of the training procedure used in which "exemplar tools, instructions, and descriptive feedback and praise emphasized generalization within tool classes” (Parsonson \& Baer, 1978, p. 378).

Holman , Goetz, and Baer (1977) trained two preschool children in block building and painting in order to increase the diversity of forms used (e.g., types of designs painted) during those activities. Results were that training on painting new painting forms did not affect demonstrations of new forms in block building. Results reported by Holman et al., (1977) were similar to those found by Parsonson and Baer (1978) in that generalization was not found to occur in the absence of procedures used to program generalization of responding across sets.

As was suggested by Holman et al., (1977) such results point out the need to program the specific type of generalization that is sought. That is, steps should be taken so that the stimuli that are to serve as discriminative stimuli for generalization responses are effective as such.

\section{Programming Generalization}

Generalization could be programmed through additional training for the purpose of facilitating contact with reinforcement for the generalization response in the presence of stimuli that are to gain discriminative control (e.g., Stokes \& Baer, 1977). In order to 
allow for the possibility of stimuli gaining discriminative control over generalization responses without such additional training, the stimuli involved in the initial training must represent the range of stimulus properties that are to be associated with correct responses (e.g., responses that may be called generalization responses).

It then follows that less generalization will be demonstrated after training in which there is less of a sameness among (1) stimuli in the presence of which the correct responses have been reinforced (i.e., training stimuli) and (2) the stimuli present in situations in which generalization is examined (i.e., generalization probes). The limited generalization reported by studies such as Parsonson and Baer (1978) may be described as a result of the training which specifically programmed only a certain type of generalization. Theoretically, the results also may be described as due to the particular range of stimuli involved in training and generalization probes (e.g., Engelmann \& Carnine, 1982).

Programming certainly is the most predictable way to occasion a desired generalization response. However, training that involves a strong sameness among (1) training stimuli and (2) generalization probe stimuli should increase the efficiency of training.

An appropriate degree of sameness among training and generalization probe stimuli may be difficult to identify prior to training since sameness among stimulus features will be responded to differently across unique student repertoires. Hence, it would be useful to described methods for arranging training so that a variety of repertoires might be more likely to respond to sameness among (1) training stimuli and (2) stimuli used in generalization probes. One such method is to training across a number 
of sets of stimuli. Training across multiple sets has the potential to increase the likelihood that the essential features of training stimuli will gain stronger control over responding since more examples and non-examples will be associated with correct responses as a result of training.

\section{Training Across Multiple Sets}

Haring (1985), examined between-sets generalization by training four children, described as persons with moderate to severe developmental disabilities, to generalize certain play responses to toys of different sets. Training involved the researcher (1) demonstrating the play response for one toy in each set of toys (e.g., toy spaceships, toy boats, and toy tanks were different sets of toys) and (2) asking the student to imitate the response using the toy. For example, the play response for spaceships was moving the toy around in the air in a circular motion and landing the spaceship at a right angle on the table.

After the initial training of the play response on one exemplar from each set, within-sets generalization training began. Within-sets generalization training consisted of training more toys in the same set (e.g., participants might be trained to demonstrate the spaceship play response on several other spaceships in the set). Within-sets generalization was demonstrated during the within-sets generalization training when the play response occurred with a member of the set before that member had been trained.

Demonstrations of between-sets generalization were described when the participant demonstrated a toy set play response for other members of the set before within-sets training occurred on the set. Thus, if the participant demonstrated the tank play response for other members of tank set before more than just one exemplar had been 
trained (i.e., within-sets training had not started on that set), the participant was described as demonstrating between-sets generalization.

Haring (1985) found that across the four participants, within-sets generalization occurred before between-sets generalization. Furthermore, between-sets generalization was found to be a function of exposure to within-sets generalization training on other toy sets.

A similar pattern of within-sets generalization occurring before between-sets generalization was found by Haring, Breen, and Laitinen (1989). Three adolescent participants, described as persons with moderate mental retardation, were trained to select age appropriate items from sets of items such as magazines, shirts, pants, and hairstyles. A match-to-sample training procedure was used in which an asterisk was associated with age appropriate items and a square was associated with age inappropriate items.

While items from a set (e.g., shirts) were trained, within-sets generalization probes were conducted on items from the same set that had not yet received training. After training had started on a certain set, a demonstration of within-sets generalization was described when an item from that set was correctly selected eight times in row before training had proceeded to that particular item.

Between-sets probes were conducted on sets from which no items had received training. A demonstration of between-sets generalization was described when an item from a set that had not been trained was correctly selected eight times in a row.

All three participants demonstrated both within-sets generalization and betweensets generalization, however, between-sets generalization was not demonstrated prior to 
at least three sets receiving training. Thus, similar to the results of Haring (1985), within set generalization occurred before between-sets generalization.

Haring et al. (1989) suggested that the results may reflect a possible extension of Engelmann and Carnine's (1982) theory, which holds that multiple exemplar training that represents the range of salient stimulus features controls within-sets responding. The extension suggested by Haring et al. (1989) suggests that, just as Engelmann and Carnine (1982) proposed that multiple exemplar training controls within-sets responding, multiple set training may control occurrences of between-sets responding (i.e., between-sets generalization). Effects of Generalization on Generalization

Effects of within-sets and between-sets generalization on transitive stimulus generalization. In addition to examining within-sets and between-sets generalization, Haring et al. (1989) also looked at transitive stimulus generalization. Two types of transitive stimulus generalization probes were conducted. The first type involved stimuli that had met the criterion of being selected correctly eight times in row as a result of training. The second type involved stimuli that had met criterion through either withinsets or between-sets generalization. Hence, selections of items that were involved in the second type of transitive stimulus generalization probes had not received reinforcement since the items were not involved in training.

Results from the transitive stimulus generalization probes showed that the first type of transitive stimulus generalization probe resulted in more demonstrations of transitive stimulus generalization. That is, probes that contained items that had been involved in training resulted in a higher than baseline number of transitive stimulus 
generalization demonstrations. Probes that contained items not used in training resulted in a number of transitive stimulus generalization demonstrations similar to baseline. Thus, demonstrations of within-sets generalization and between-sets generalization alone did not facilitate transitive stimulus generalization. In other words, demonstrating within-sets or between-sets generalization (i.e., an extension of responding from training stimuli to stimuli not involved in training) did not facilitate the transitive relation among stimuli. That result brings up a question concerning whether or not transitive stimulus generalization would effect demonstrations of within-sets and between-sets generalization.

Transitive stimulus generalization effect on extensions of responding. Training that facilitates transitive stimulus generalization has been shown to affect extensions of responding to other contexts. Lane and Critchfield (1998) used a match-to-sample procedure as a means of facilitating an extension of a reading skill from the training context to another setting for two adolescents with Down syndrome. In the match-tosample training, samples were either the spoken word "vowel" or "consonant" and comparisons were printed vowels and consonants. After training was shown to facilitate transitive stimulus generalization, generalization probes were conducted to examine an extension of responding to another context. Participants were given printed words which contained the vowels used in training and were asked to mark the vowels. Responding was shown to generalize to the new context for both participants. Thus, training that occasioned transitive stimulus generalization also was shown to occasion an extension of responding to another context. 
Similar results were found by Lynch and Cuvo (1995) after using a match-tosample procedure to train seven fifth and sixth grade students identified as having difficulty with tasks involving fractions and decimals. Participants were trained to (1) select fraction pictures (Set B stimuli) in the presence of ratios (Set A stimuli) and (2) select decimals (Set C stimuli) in the presence of fraction pictures (Set B stimuli). That is, participants were trained on $\mathrm{AB}$ and $\mathrm{BC}$ relations. After training, all participants demonstrated transitive stimulus generalization by correctly selecting decimals in the presence of ratios (AC discriminations) and ratios in the presence of decimals (CA discriminations).

Generalization tests for an extension of responding to similar stimuli not involved in the training tested selections of novel decimals (an extension of Set C ) in the presence of novel ratios (an extension of Set A) and selections of novel ratios in the presence of novel decimals. Thus, the generalization probes tested (1) an extension of responding to stimuli that were part of larger sets involved in training (i.e., within-sets generalization) on (2) an emergent transitive relation (i.e., an untrained yet demonstrable relation).

Results showed that only three of the seven participants demonstrated the extension of responding examined in the generalization probes. Since generalization probes tested an extension of responding to stimuli that were part of larger sets by requiring participants also to respond to the untrained transitive relation (i.e., the relation was not the subject of reinforced trials), generalization tests may have been more difficult.

Perhaps tests examining only an extension of responding to stimuli that were part of larger sets (i.e., just within-sets generalization) would have returned more 
demonstrations of generalization. Thus, even though the generalization results occurred for only three participants, the results offer strong support to the idea that training which occasions transitive stimulus generalization also will occasion other types of generalization.

Possible transitive stimulus generalization effects on within-sets and between-sets generalization. Although the generalization of selections of stimuli in training to other contexts was not shown to be a function of transitive stimulus generalization in Lane and Critchfield (1998) and Lynch and Cuvo (1995), the training which facilitated transitive stimulus generalization also facilitated generalization across contexts. Hence, the result suggests that training that facilitates transitive stimulus generalization may also facilitate other types of generalization. This raises an interesting question about possible effects of transitive stimulus generalization on demonstrations of within-sets and between-sets generalization. Perhaps generalization results following training that facilitates transitive stimulus generalization might not follow the pattern of within-sets generalization occurring before between-sets generalization. If within-sets generalization and betweensets generalization both were to occur directly after training, a training procedure with great deal of utility would be described.

Match-to-sample training has been described in previous paragraphs as training that has the potential to occasion transitive stimulus generalization. In the following section, match-to-sample training effects on transitive stimulus generalization are discussed for the purpose of describing training that may most efficiently occasion transitive stimulus generalization. 


\section{Match-to-Sample Training Effect on Transitive Stimulus Generalization}

Transitive stimulus generalization has been shown to be effected by the type of MTS training (one-to-many or many-to-one) used. In the following paragraphs, the effect that one-to-many (OTM) training and many-to-one (MTO) training have been shown to have on demonstrations of transitive stimulus generalization are described.

Saunders, Drake, and Spradlin (1999) used five sets of arbitrary stimuli to train typically developing preschool aged children in either a OTM direction or a MTO direction. Two of six children who were trained in the OTM direction met the criterion of 83\% response accuracy on transitive relations. However, five of five met the criterion for response accuracy on transitive relations after training in the MTO direction.

Among adult and adolescent participants with mild mental retardation, positive outcomes on transitive stimulus generalization have been found to be more likely after MTO training than OTM training. Fields and Hobbie-Reeve (1999) found that following MTO versus OTM training involving seven stimulus sets, college students were more likely to meet the criterion of 95\% response accuracy on transitive relations.

Number of stimulus sets. Results obtained by Saunders, Drake, and Spradlin (1999) and Fields and Hobbie-Reeve (1999) suggest that when several stimulus sets are used (e.g., more than 3 sets), transitive stimulus generalization may be affected by training direction. Further, studies examining conditional discriminations that used fewer stimulus sets (e.g., 3 sets) suggest that both OTM (Eikeseth \& Smith, 1992; Innis, Lane, Miller, \& Critchfield, 1998; Pilgrim \& Galizio, 1990; Sidman, Kirk, \& Willson-Morris, 1985) and MTO (Williams, Saunders, Saunders, \& Spradlin, 1995) training directions lead to positive outcomes on transitive stimulus generalization. 
Simple discriminations. Theoretically, the MTO training direction, when compared to the OTM training direction, does appear to be more likely to lead to positive outcomes on tests for transitive relations among stimuli as a result of training. Possible training direction differences have been attributed to the number of simple discriminations that are required during training (Barnes, 1994; Saunders \& Green, 1999; Spradlin \& Saunders, 1986). More specifically, in contrast to MTO training, OTM training does not require participants to make all the simple discriminations between stimuli that are later required in tests for transitive relations.

As Sidman (1990) and Saunders and Green (1999) discussed, when the selection of a certain comparison stimulus in the presence of a certain sample stimulus is followed by a positive consequence, the selection of the comparison stimulus may be more likely in the presence of the sample stimulus than in the presence of another sample stimulus. If such selection is demonstrated, a simple discrimination is shown. When a simple discrimination is made between stimuli presented at the same time, a simultaneous simple discrimination is shown. Thus, simultaneous simple discriminations may occur in matchto-sample training between comparison stimuli presented in the same trial and between individual comparison stimuli and the sample stimulus of the trial. When simple discriminations are made between different sample stimuli on successive trials, a successive simple discrimination is shown. Thus, successive simple discriminations may occur in match-to-sample training between the sample stimuli presented on successive trials.

Corresponding stimuli (e.g., C1 and B1) from comparison sets are presented successively as comparison stimuli in the OTM training direction. However, it is 
assumed that making such successive simple discriminations would not be as likely during OTM training since corresponding comparison stimuli are not directly conditioned as discriminative stimuli (i.e., stimuli that are associated with the availability of reinforcement) for a certain corresponding sample stimulus (e.g., A1). In the MTO direction, corresponding stimuli (e.g., C1 and B1) from sample sets are directly conditioned as discriminative stimuli for a certain corresponding comparison stimulus (e.g., A1) (Barnes, 1994; Saunders \& Green, 1999; Spradlin \& Saunders, 1986). Hence, MTO training compared to OTM training may present more simple discriminations that are later required in tests of transitive stimulus generalization (e.g., C2B2 or B3C3).

Based on the discussion in the previous paragraphs, many-to-one training appears to lead consistently to positive outcomes on tests for transitive relations among stimuli as a result of training. Thus, many-to-one training would be a good type of training to use to examine the occurrence of within-sets generalization and between-sets generalization after transitive stimulus generalization is shown.

Statement of the Problem

As was described previously in the present paper, between-sets generalization has been shown to be dependent on within-sets generalization training. A question pertaining to a functional relation between transitive stimulus generalization and other types of generalization (e.g., within-sets or between-set generalization) remains. There is, however, evidence that training which occasions transitive stimulus generalization also may lead to other types of generalization without additional programming.

Training for the purpose of facilitating an extension of responding from trained material to other material has been referred to as teaching for transfer (Good \& Brophy, 
1995). As Good and Brophy (1995) described, Jerome Bruner stressed that in order for transfer to occur, students first must have an adequate amount of base knowledge. With the proper repertoire, students then may transfer trained associations among stimuli to other situations that are likely to involve new associations among new stimuli (Bruner, 1960). In the present study, training that facilitates transfer from training to other settings is training that occasions transitive stimulus generalization, within-sets generalization, or between-sets generalization.

In order to extend the current generalization literature, an examination of a possible functional relation between transitive stimulus generalization and either withinsets or between-sets generalization is needed. Such an examination of generalization has implications for both basic and applied research.

Implications for basic work are found in the examination of a functional relation between types of generalization. From an applied stance, a tool with a great deal of potential efficiency for altering repertoires may be described. That is, if the training method used facilitates transitive stimulus generalization and also leads to the demonstration of within-sets generalization and between-sets generalization without additional programming, an efficient tool will be described. Such a tool would be efficient in that training involving a relatively small number of stimuli would be shown to occasion an extension of responding to a relatively large number of stimuli.

Many-to-one match-to-sample training has been shown to be an efficient training procedure for facilitating transitive stimulus generalization. Therefore, many-to-one training would be a reasonable training procedure for an examination of possible 
functional relation between transitive stimulus generalization and either within-sets or between-sets generalization. 
Chapter 3: Method

\section{Research Question and Procedures}

In the present study, a many-to-one match-to-sample procedure was used to train four typically developing five-year-old children to select fraction ratios in the presence of fraction pictures. After the match-to-sample training, testing occurred for transitive stimulus generalization, within-sets generalization, and between-sets generalization. Transitive stimulus generalization training then was begun while testing continued on within-sets generalization and between-sets generalization. That was done in order to determine whether training on transitive stimulus generalization would affect demonstrations of either within-sets or between-sets generalization (i.e., whether withinsets or between-sets generalization is dependent on transitive stimulus generalization).

Following accurate responding on transitive stimulus generalization test discriminations, within-sets generalization training was added to transitive stimulus generalization training. During the within-sets generalization training, between-sets generalization was tested. That was done in order to determine whether the addition of within-sets generalization training to transitive stimulus generalization training would affect demonstrations of between-sets generalization.

\section{Research Questions}

After training typically developing five-year-old children to select fraction ratios in the presence of fraction pictures using a many-to-one match-to-sample procedure, (1) will increased training on transitive stimulus generalization increase the number of demonstrations of within-sets and between-sets generalization and (2) will the addition of 
within-sets generalization training to transitive stimulus generalization training increase the number of demonstrations of between-sets generalization? Variables

The dependent variables were (1) the number of demonstrations of transitive stimulus generalization, within-sets generalization, and between-sets generalization after initial match-to-sample training, (2) the number of demonstrations of within-sets and between-sets generalization after transitive stimulus generalization training, and (3) the number of demonstrations of between-sets generalization after within-sets generalization training. The independent variables were (1) initial training, (2) transitive stimulus generalization training, and (3) within-sets generalization training.

\section{Participants and Setting}

Participants were four typically developing five-year-old children attending Kindergarten in a public school close to West Virginia University. The legal guardians of potential participants were contacted by the researcher through the classroom teachers. Data collection sessions took place in the school's facilities and lasted approximately 15 minutes. Sessions took place at a table with two chairs. The table was located outside the participant's classroom. The first four potential participants who returned a signed consent form were asked to participate.

Participant 1 had a total of four sessions, Participant 2 had a total of seven sessions, and Participants 3 and 4 each had a total of eight sessions. Sessions took place near the beginning of the school day, and individual sessions for each participant were one or two days apart. 
Pretest

Participants were given a pretest to check the likelihood that the participant already had in his or her repertoire the discriminations that were to be trained and tested. In order to check for this, a baseline block was administered as a pretest. Baseline blocks are described below. During the pretest, attempt cards were presented as described in the Baseline section of this paper.

In the pretest, participants were required to fail at least $2 / 3$ of the attempt cards (i.e., no more than 16 of the 24 attempt cards passed) in order to continue with the study. The pretest also served as the start of baseline data prior to initial training.

\section{Stimulus Sets}

Initial training. Three, 3-member sets of stimuli were used in training. Set A (A1, A2, A3) stimuli were fractions in ratio form. Stimuli from Set B (B1, B2, B3) were fraction pictures of groups of circles in which the fraction numerator was represented by filled-in circles. Stimuli from Set C (C1, C2, C3) were fraction pictures of groups of circles in which the fraction numerator was represented by non-filled-in circles. Figure 2 contains the sets of stimuli used in initial training. Training was done in a many-to-one direction, meaning that sample stimuli came from Set B or Set C and comparison stimuli were the entire Set A. Thus, BA and CA discriminations were trained.

Transitive stimulus generalization. Transitive stimulus generalization is described as an extension of responding from trained relations among stimuli to another relation among the stimuli that is occasioned by a shared intermediary relation among the stimuli. Transitive stimulus generalization probes were tests for an emergent transitive relation among stimuli involved in initial training. Since initial training involved BA and CA 
Set A
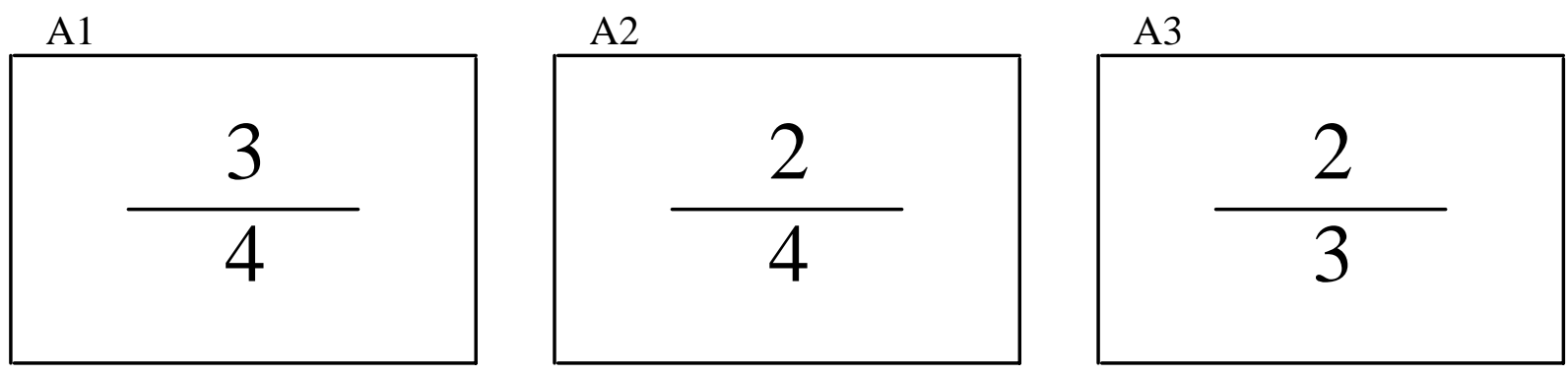

Set B
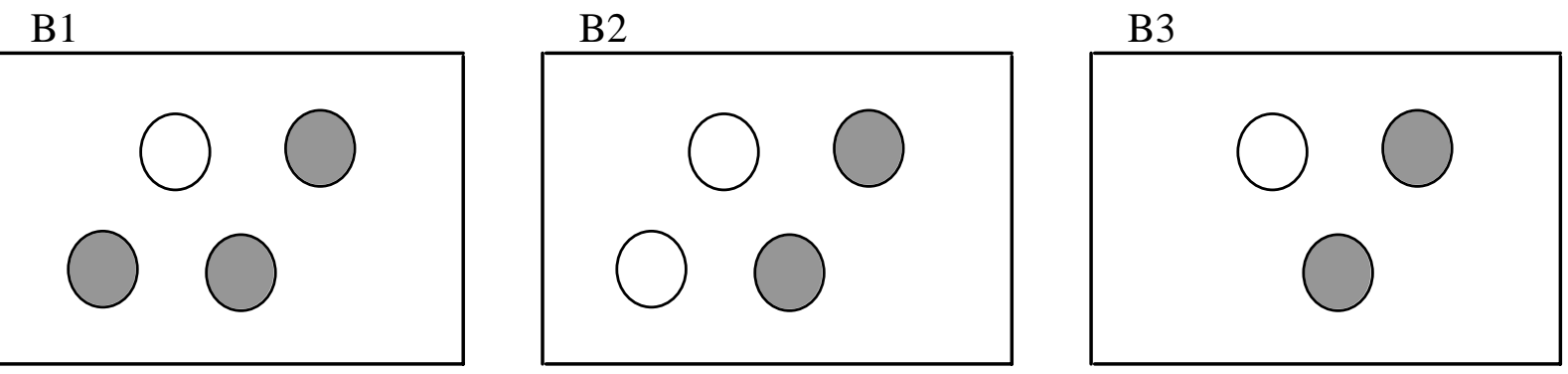

Set C
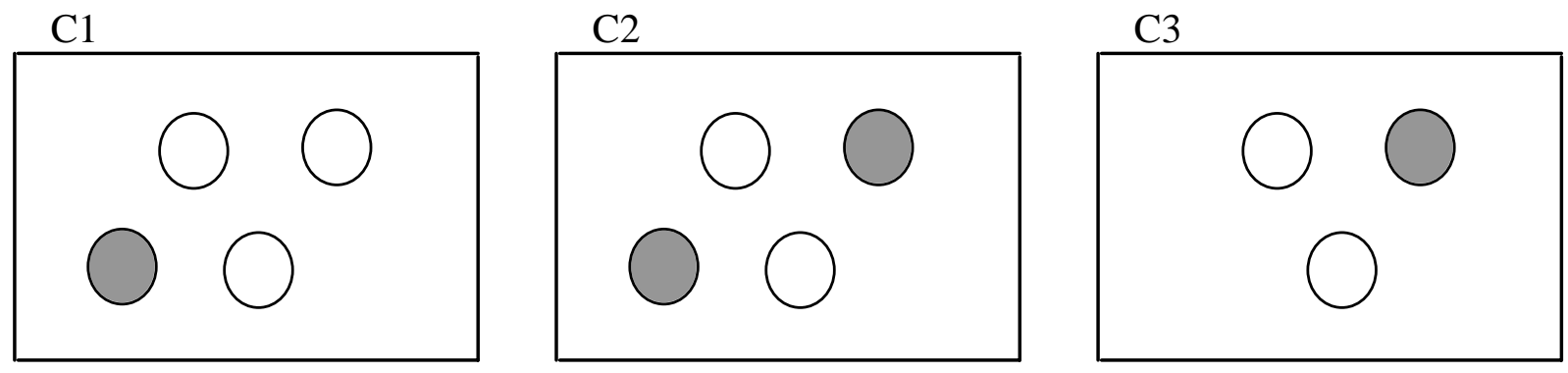

Figure 2. Stimulus sets used in initial training and transitive stimulus generalization probes (IT and TSG). 
discriminations, $\mathrm{BC}$ and $\mathrm{CB}$ discriminations tested an emergent transitive relation. Hence, transitive stimulus generalization probes were tests of $\mathrm{BC}$ and $\mathrm{CB}$ discriminations.

Within-sets generalization. Within-sets generalization is described as an extension of responding from sets involved in initial training to sets containing stimuli that are part of larger sets that include the stimuli used in initial training. Within-sets generalization probes involved testing stimuli from Set X (X1, X2, X3), Set Y (Y1, Y2, Y3), and Set Z (Z1, Z2, Z3) in a many-to-one direction, meaning that sample stimuli came from Set Y or Set Z and comparison stimuli were the entire Set X. Stimuli of Set X were part of a larger set including stimuli from the training Set A, stimuli of Set Y were part of a larger set including stimuli from the training Set B, and stimuli of Set $\mathrm{Z}$ were part of a larger set including stimuli from the training Set C. Hence, Set X stimuli were fraction ratios, Set Y stimuli were fraction pictures of groups of circles in which the fraction numerator was represented by filled-in circles, and Set C stimuli were fraction pictures of groups of circles in which the fraction numerator was represented by nonfilled-in circles. Stimuli involved in within-sets generalization probes had not been used in initial training. Figure 3 contains the sets of stimuli used in within-sets generalization probes.

Between-sets generalization. Between-sets generalization is described as an extension of responding from sets that were involved in initial training to different sets that do not contain the stimuli from sets used in initial training. That is, between-sets generalization is described when participants respond to stimuli from sets that are not part of larger sets involved in initial training. Between-sets generalization probes involved 
Set X
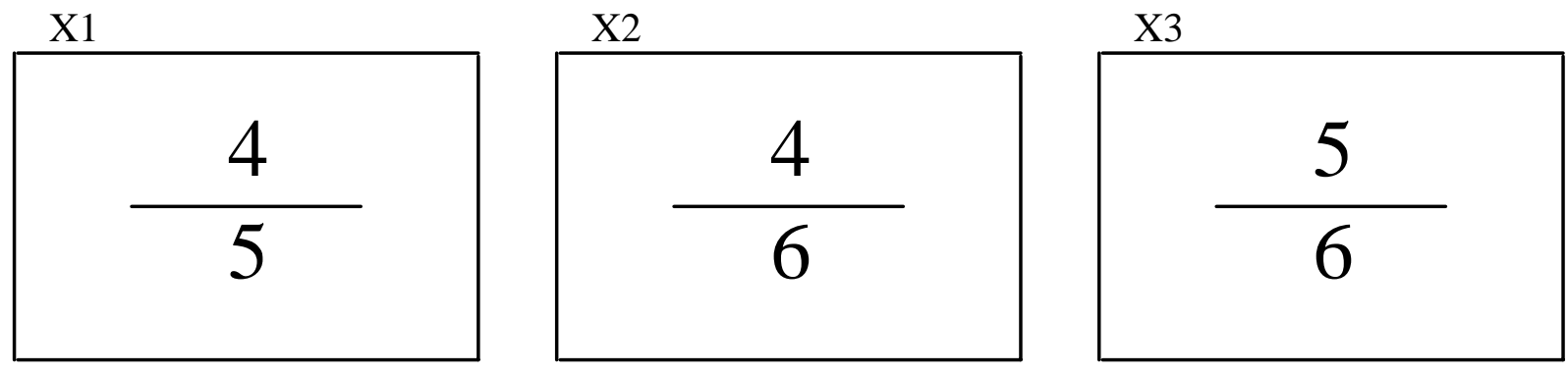

Set Y
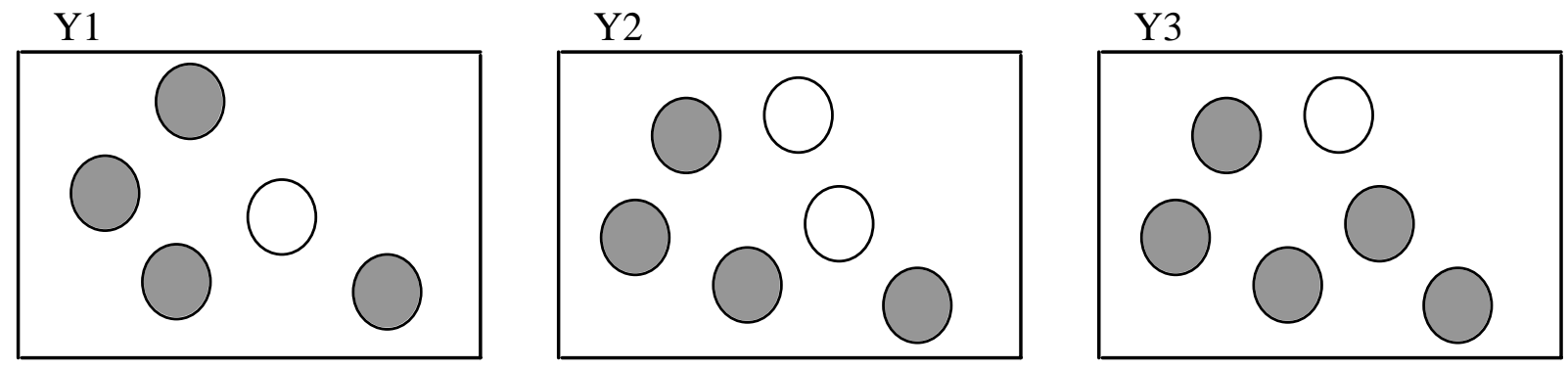

Set Z
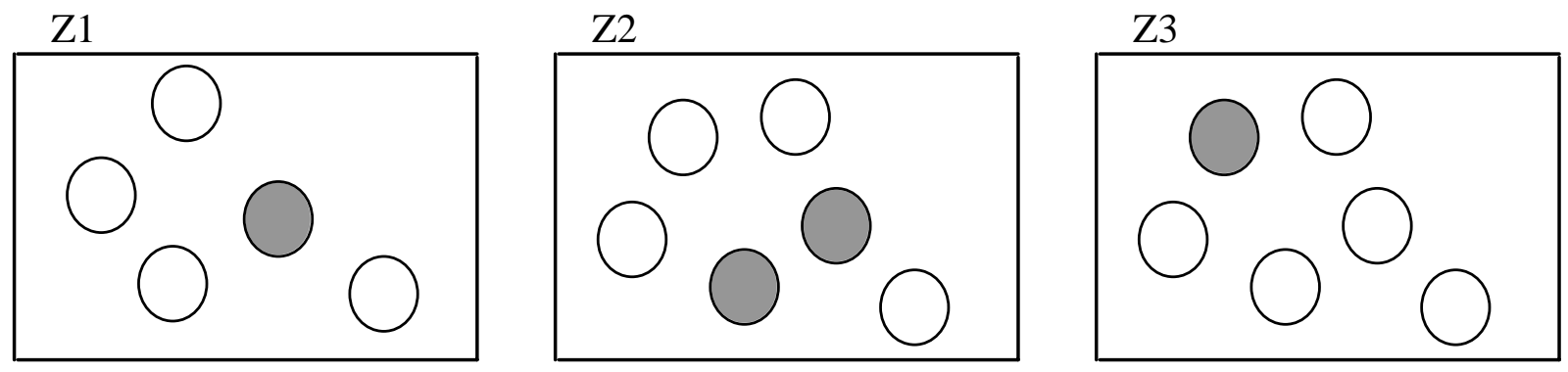

Figure 3. Stimulus sets used in within-sets generalization (WSG) probes. 
testing stimuli from Set D (D1, D2, D3), Set E (E1, E2, E3), and Set F (F1, F2, F3) in a many-to-one direction, meaning that sample stimuli came from Set E or Set F and comparison stimuli were the entire Set D. Figure 4 contains the sets of stimuli used in between-sets generalization probes.

Stimuli of Set D were fraction ratios used in the within-sets generalization probes. Stimuli of Set E were fraction pictures of rectangles partitioned into equal sized sections in which the fraction numerator was represented by filled-in sections. Stimuli of Set F were fraction pictures of rectangles partitioned into equal sized sections in which the fraction numerator was represented by non-filled-in sections. Set E stimuli and Set F stimuli were similar to the sets of sample stimuli used in training (i.e., Set B and Set C) and within-sets generalization probes (i.e., Set Y and Set Z ), but were not part of those sets since they did not share all essential features (i.e., Set E and Set F stimuli were not fraction pictures of circles).

General Procedure

Rewards. Participants were told that at the end of each session they would be allowed to select a sticker from an assortment of stickers in a small box. In order for participants to monitor how close they were to being able to select a sticker, they were given a scorecard at the start of each session that had to be filled with stars before a sticker could be selected. Stars were drawn by the researcher on a scorecard that remained in the participant's sight throughout data collection.

Attempts and trials. Throughout all phases of the current study, stimuli were presented on attempt cards. Attempt cards used in all phases had one sample stimulus printed on them with three comparison stimuli printed under the sample. Each conditional 
Set D
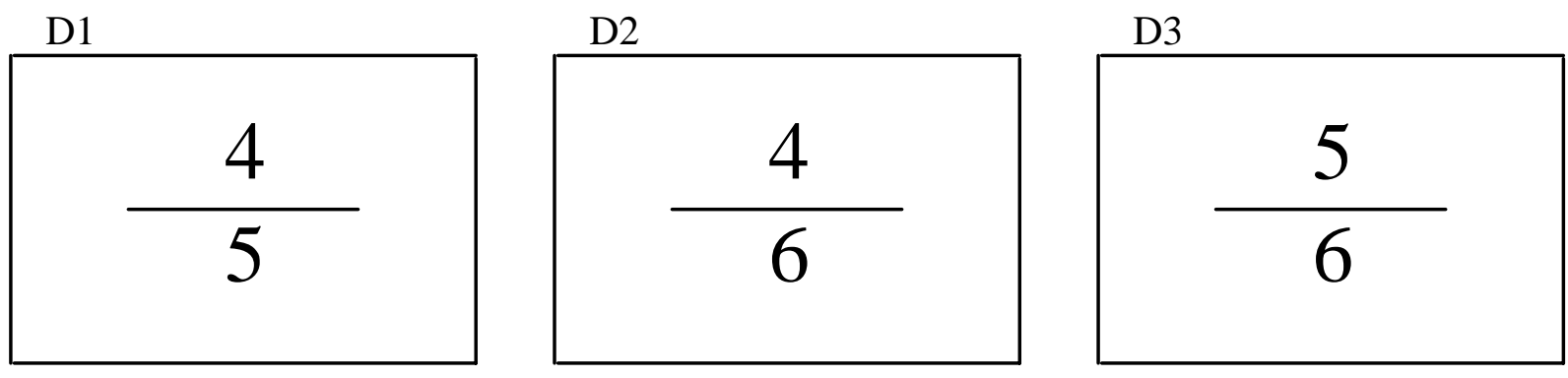

Set E
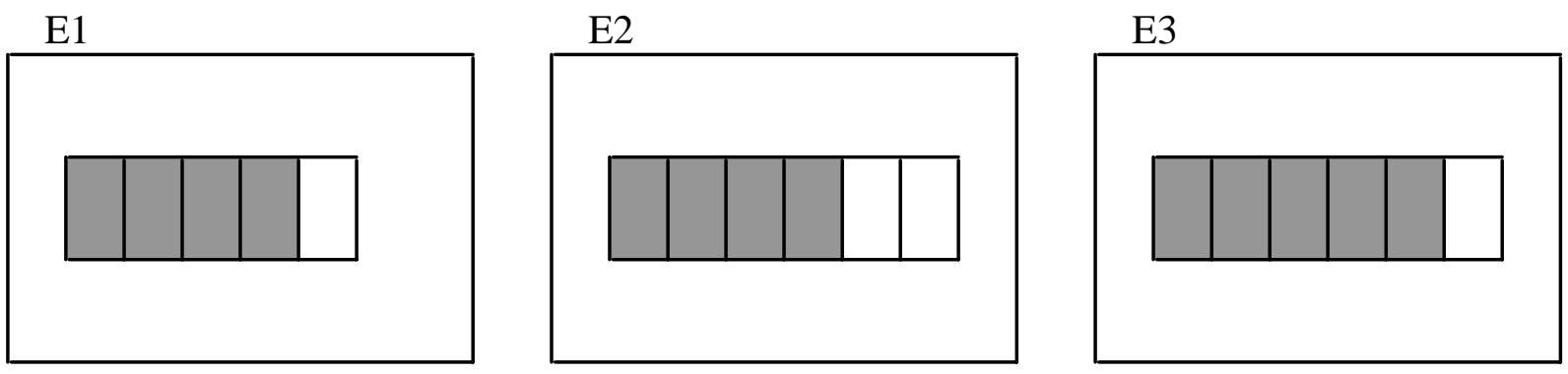

Set F
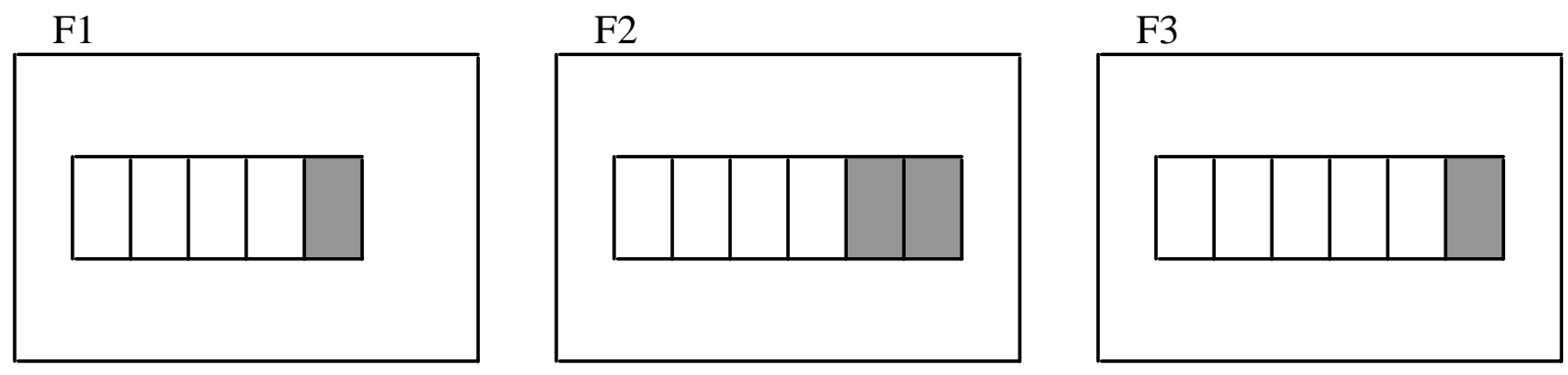

Figure 4. Stimulus sets used in between-sets (BSG) generalization probes. 
discrimination (e.g. B1A1) had six attempt cards. The attempt cards for a certain conditional discrimination differed only in the order that the comparison stimuli appeared on the card. For example, the order of comparison stimuli on one B1A1 attempt card was A1 A2 A3 and the order on a different B1A1 attempt card was A2 A1 A3. See Figure 5 for samples of attempt cards.

The first trial on a certain attempt card occurred when the participant selected a comparison stimulus. If the selection was incorrect, the second trial on the same attempt card occurred when the participant selected another comparison stimulus. An attempt was considered passed only if the correct comparison was selected on the first trial of the attempt; otherwise, the attempt was considered failed.

\section{Initial Training (Phase A)}

Training was done in two parts. In the first part of training, BA and CA discrimination were trained separately. In the second part of training BA and CA discrimination were trained together. During both parts of training, participants were told after each trial on an attempt card if their selection of a comparison stimulus was correct or incorrect. Data on the number of attempts and trials on each conditional discrimination were recorded on a scorecard by the researcher after each attempt. See Appendix A for sample part one and part two initial training scorecards. Scorecards were positioned so that participants could not see what was recorded on them.

Part one training. During part one of training, participants first were trained only on BA conditional discriminations (B1A1, B2A2, B3A3), then only on the CA conditional discriminations (C1A1, C2A2, C3A3). Since each conditional discrimination 


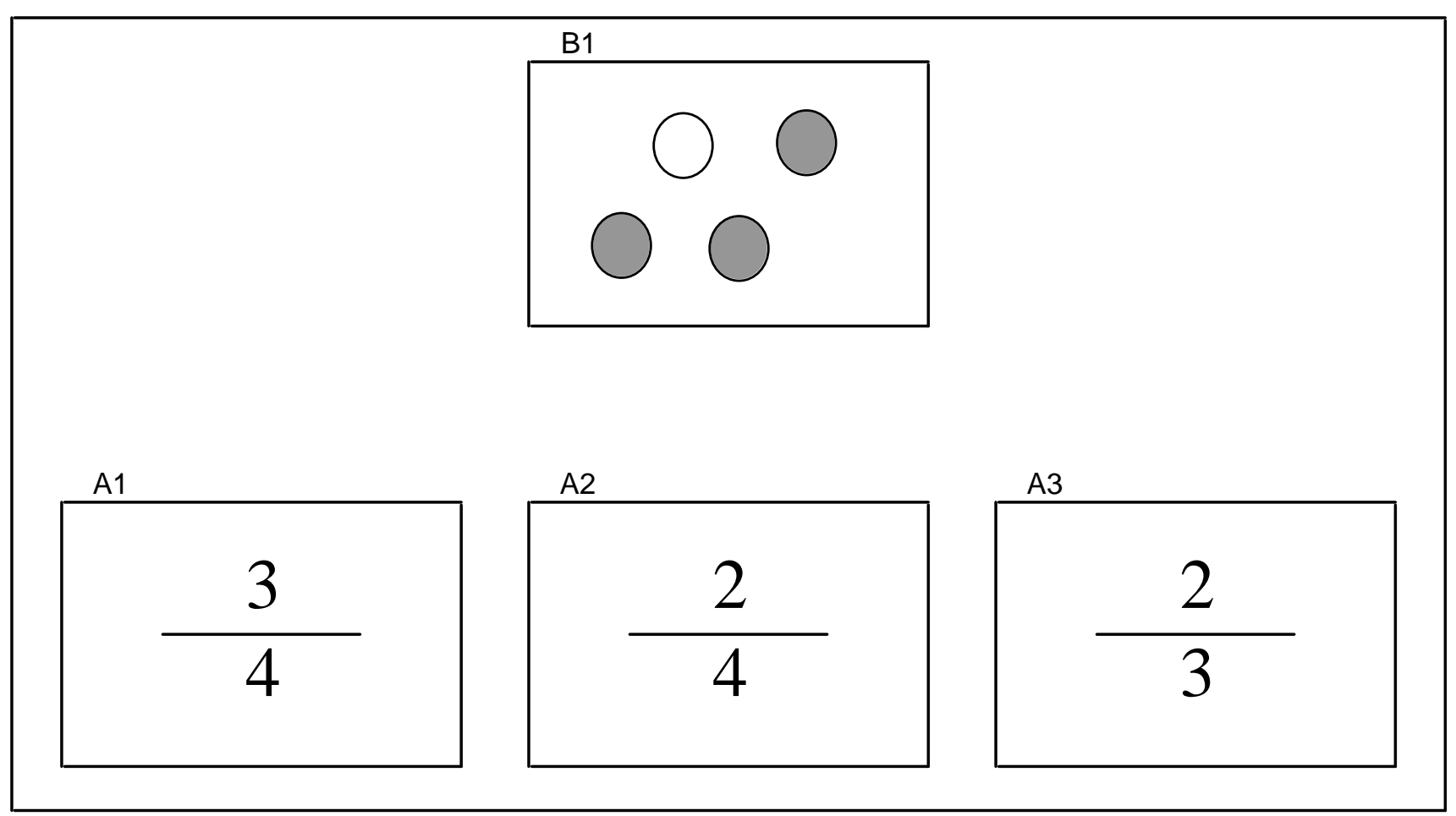

B1A1 Attempt Card

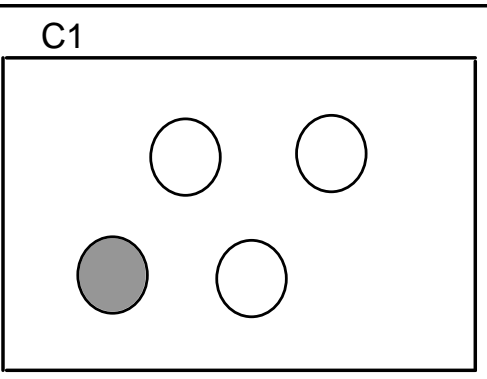

A1

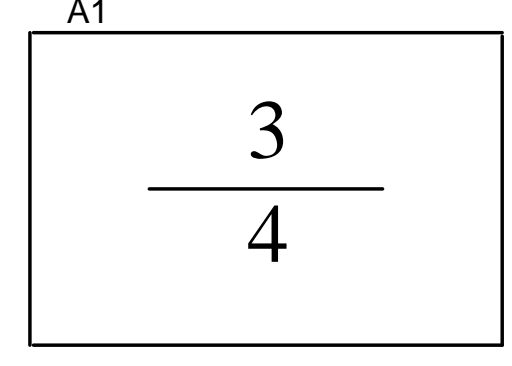

A2

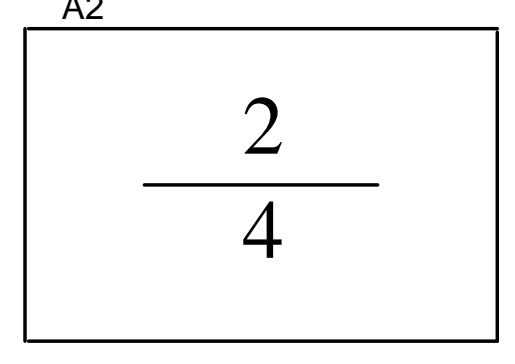

A3

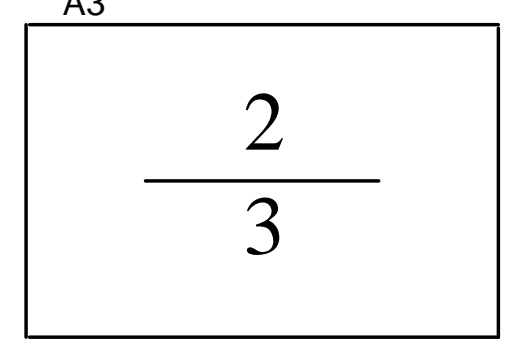

C1A1 Attempt Card

Figure 5. Samples of attempt cards. 
had six attempt cards, a total of 18 attempt cards were involved in training each type of conditional discrimination (e.g., BA).

Part one training attempts. The 18 attempt cards for the first three discriminations trained in part one (i.e., B1A1, B2A2, B3A3) were shuffled together. The first attempt card then was presented. The researcher asked the participant to select a comparison stimulus on the attempt card by saying, "Pick a picture from this set [pointing to the comparison set] that goes with this picture [pointing to the sample stimulus].” If the participant asked for help, said that he or she didn't know which comparison was correct, or did not respond after approximately ten seconds, the researcher said, "Take a guess, you'll have another chance after this.” Facial expressions and gestures that could have indicated that the participant did not know the answer were ignored.

If the participant pointed to the correct comparison stimulus on the first trial of an attempt card, the researcher said “Good.” That attempt then was recorded on a scorecard as passed. If the participant pointed to an incorrect comparison on the first trial of an attempt card, the researcher said, “No, try again.” The second trial on the same attempt card then occurred when the participant selected another comparison stimulus. Trials on the same attempt card were continued until the participant selected the correct comparison.

Once the participant selected the correct comparison stimulus after selecting an incorrect comparison, the researcher said “Good.” That attempt then was recorded on a scorecard as failed (since it was not passed on the first trial), and the number of trials that were needed also were recorded. 
After the correct comparison stimulus was selected on an attempt card, that card was placed at the bottom of the stack. The next attempt card in the stack then was presented. Attempt cards for the same conditional discrimination (e.g., B1A1) were not presented back-to-back. Thus, if the conditional discrimination on an attempt card was the same as the last discrimination presented, that card was shuffled back into the stack.

Part one training condition criteria. Training on an individual conditional discrimination (e.g., C2A2) was considered complete when the participant passed three consecutive attempts on the conditional discrimination. If an attempt on a certain conditional discrimination was failed after a previous attempt had been passed, the number of attempts passed on that conditional discrimination was reset to zero. Once complete, the conditional discrimination was dropped from the condition, and the participant earned a star. Hence, in part one of initial training, participants earned a total of 6 stars (one star for each BA and CA discrimination trained).

Once a conditional discrimination was dropped from the condition, it still could be presented for the purpose of preventing two of the same conditional discriminations from being presented back-to-back. If an attempt on a conditional discrimination that had been dropped (i.e., previously passed on three consecutive attempts) was failed on a later presentation, the number of attempts passed on that conditional discrimination was not reset to zero.

Part two training. During part two of training, participants were given a mix of BA conditional discriminations (B1A1, B2A2, B3A3) and CA conditional discriminations (C1A1, C2A2, C3A3). Since each conditional discrimination had six attempt cards, a total of 36 attempt cards were involved in the mixed training trials. 
Part two training attempts. The 36 attempt cards for the six conditional discriminations trained in part two were shuffled together. Attempt cards were presented in the same way they were presented during part one of training. As before, scorecards were positioned so that participants could not see what was recorded on them.

Part two training condition criteria. The condition criteria were identical to those used in part one. Thus, training on an individual conditional discrimination (e.g., C3A3) was complete when a participant passed three consecutive attempts on the conditional discrimination. Once complete, the conditional discrimination was dropped from the condition, and the participant earned a star. Hence, in part two of initial training, participants earned a total of 6 stars (one star for each BA and CA discrimination trained).

Baseline (Phase B)

The purpose of the baseline was to record the number of demonstrations of transitive stimulus generalization, within-sets generalization, and between-sets generalization after initial training. During baseline, transitive stimulus generalization probes, within-sets generalization probes, and between-sets generalization probes were presented in blocks of attempt cards.

Transitive stimulus generalization probes were attempt cards of BC or CB discriminations. Thus, three BC discriminations (i.e., B1C1, B2C2, B3C3) and three CB discriminations (i.e., C1B1, C2B2, C3B3) were involved in transitive stimulus generalization probes.

Within-sets generalization probes were presentations of attempt cards that had sample stimuli from Set Y or Set Z and comparison stimuli as the entire Set X. Thus, 
three YX discriminations (i.e., Y1X1, Y2X2, Y3X3) and three ZX discriminations (i.e., Z1X1, Z2X2, Z3X3) were involved in within-sets generalization probes.

Between-sets generalization probes were presentations of attempt cards that had sample stimuli from Set E or Set F and comparison stimuli as the entire Set D. Thus, three ED discriminations (i.e., E1D1, E2D2, E3D3) and three FD discriminations (i.e., F1D1, F2D2, F3D3) were involved in between-sets generalization probes.

Baseline blocks. Each baseline block contained one attempt card for each discrimination involved in the transitive stimulus generalization probes, the within-sets generalization probes, and the between-sets generalization probes. That is, each discrimination involved in a certain type of generalization was presented just once in each block. Since there were six discriminations involved in each type of generalization, there were 18 generalization probes in each block.

Blocks were arranged such that a transitive stimulus generalization attempt card was presented followed by a within-sets generalization card, followed by a between-sets generalization card, which was followed by an attempt card used in initial training. Thus, each baseline block contained a total of 24 attempt cards. Of the 24 attempt cards in a baseline block, 18 of the attempt cards were generalization probes, and 6 of the attempt cards were attempt cards used in initial training. Blocks differed from one another in (1) the placement of specific generalization discriminations in each block (e.g., in one block, Y2X2 may have been the first within-sets attempt card presented, but in another block it may have been the last within-sets attempt card presented) and (2) the attempt cards used for specific generalization discriminations (e.g., the order of comparison stimuli on the 
C2B2 attempt card of one block was different in the following block). See Appendix B for sample baseline blocks.

Baseline attempts. During baseline the only arranged consequence for correct or incorrect selections of comparison stimuli on the attempt cards was the presentation of another attempt card. That is, each attempt card had only one trial. If the correct comparison was selected on that trial, the attempt was marked as passed on a scorecard and the next attempt card was presented. If an incorrect comparison was selected on that trial, the attempt was marked as failed, and the next attempt card was presented. As before, scorecards were positioned so that participants could not see what was recorded on them. After each block, the participant earned a star on his or her scorecard. See Appendix C for sample baseline scorecards.

As described in the previous paragraph, participants were not told whether or not their selections of comparison stimuli were correct on attempts during baseline. In order to avoid a situation in which the researcher would be completely silent during baseline data collection (a situation that may be uncomfortable for some participants), the researcher said, “Here’s the next card” or “ Let’s try this one now” after every few attempt cards.

Initial training attempts in baseline. The presentation of the initial training attempt cards during baseline served as a way to determine whether or not participants continued to make accurate selections during baseline. That is, if outcomes on generalization attempt cards were poor while initial training discriminations remained accurate, it would be unlikely that the generalization outcomes were due only to motivational variables (e.g., satiation with reinforcement available). Responding was 
considered accurate on initial training attempt cards that were presented across baseline blocks if at least 2/3 of the initial training attempt cards were passed (e.g., if one baseline block were used, at least 4 of the 6 initial training attempt cards would have to be passed).

Transitive Stimulus Generalization Training (Phase C)

The purpose of the transitive stimulus generalization training phase was to examine the effect that the direct training on the transitive relation had on demonstrations of within-sets and between-sets generalization. Transitive stimulus generalization training was applied to baseline blocks. Selections of comparison stimuli on all attempt cards presented during the transitive stimulus generalization training phases were recorded as passed or failed on a scorecard that was positioned so that participants could not see what was recorded on them. After each block, the participant earned a star on his or her scorecard.

Phase C blocks. Transitive stimulus generalization training was applied to baseline blocks, meaning that baseline blocks were presented as they were during baseline. Procedures for presenting attempt cards in the baseline blocks remained the same during transitive stimulus generalization training, with the exception of the presentation of attempt cards for BC and CB discriminations (i.e., the transitive stimulus generalization attempt cards). During the transitive stimulus generalization training phase, $\mathrm{BC}$ and $\mathrm{CB}$ discriminations were trained. When $\mathrm{BC}$ and $\mathrm{CB}$ attempt cards were presented in a block, the participant was told whether or not his or her selection of comparison stimulus was correct or incorrect. That is, transitive stimulus generalization was directly trained. 
Application of transitive stimulus generalization training. If the participant pointed to the correct comparison stimulus on the first trial of a $\mathrm{BC}$ or $\mathrm{CB}$ attempt card, the researcher said "Good.” That BC or CB attempt then was recorded on a scorecard as passed. If the participant pointed to an incorrect comparison on the first trial of the attempt card, the researcher said, "No, try again." Once the participant selected the correct comparison stimulus after selecting an incorrect comparison, the researcher said “Good.” That BC or CB attempt then was recorded on a scorecard as failed (since it was not passed on the first trial).

Initial training attempts in Phase $C$. During the transitive stimulus generalization training phase, the initial training discriminations that were presented in blocks served as a way to determine whether or not participants continued to make accurate selections during the phase. That is, if outcomes on within-sets and between-sets generalization discriminations were poor while initial training discriminations remained accurate, it would be unlikely that the generalization outcomes were due only to motivational variables (e.g., satiation with reinforcement available). Within-Sets Generalization Training (Phase D)

The purpose of the within-sets generalization training phase was to examine the effect that the direct training on within-sets generalization discriminations had on demonstrations of between-sets generalization. Within-sets generalization training was applied to baseline blocks. Selections of comparison stimuli on all attempt cards presented during within-sets generalization training phases were recorded as passed or failed on a scorecard that was positioned so that participants could not see what was recorded on them. After each block, the participant earned a star on his or her scorecard. 
Phase D blocks. Procedures used during within-sets generalization training were identical to procedures used during transitive stimulus generalization training with the addition that training also was supplied to the $\mathrm{YX}$ and $\mathrm{ZX}$ discriminations. That is, when YX and ZX attempt cards were presented in a block, the participant was told whether or not his or her selection of comparison stimulus was correct or incorrect.

Application of within-sets generalization training. If the participant pointed to the correct comparison stimulus on the first trial of a YX or ZX attempt card, the researcher said “Good.” That YX or ZX attempt then was recorded on a scorecard as passed. If the participant pointed to an incorrect comparison on the first trial of the attempt card, the researcher said, “No, try again.” Once the participant selected the correct comparison stimulus after selecting an incorrect comparison, the researcher said “Good.” That YX or ZX attempt then was recorded on a scorecard as failed (since it was not passed on the first trial).

\section{Research Design}

The questions addressed in the current study are as follows. (1) Will increased training on transitive stimulus generalization increase the number of demonstrations of within-sets and between-sets generalization? (2) Will the addition of within-sets generalization training to transitive stimulus generalization training increase the number of demonstrations of between-sets generalization? In order to answer those questions, participants were exposed to a pretest (start of baseline data), Phase A (initial training), Phase B (baseline), Phase C (transitive stimulus generalization training), and Phase D (within-sets generalization training). 


\section{Summary of Consequences}

The following is a summary of consequences for comparison selections in baseline blocks used in different phases. During the pretest, the only arranged consequence for participants' selections of comparison stimuli on attempt cards was the presentation of the next attempt card. During Phase B (baseline), attempt cards were presented as they were in the pretest.

During Phase C (transitive stimulus generalization training), attempt cards were presented as they were in Phase B with the addition of training following comparison selections on attempt cards used for transitive stimulus generalization probes. During Phase D (within-sets generalization training), attempt cards were presented as they were in Phase $\mathrm{C}$ with the addition of training following comparison selections on attempt cards used for within-sets generalization probes.

\section{Data Collection Conditions}

Data were collected in sessions that were approximately 15 minutes in length. Each data collection session involved one data collection condition. See Figure 6 for the three data collection conditions that were used. In Condition 2 and Condition 3, if Phase A (initial training) was returned to, only part two of initial training was used (i.e., training on the mixture of BA and CA discriminations).

Repeating data collection conditions. Each participant was exposed to Condition 1 in his or her first session. Subsequent sessions involved either Condition 2 or Condition 3. Following Condition 1, consecutive sessions involved Condition 2 until responding in Phase C (transitive stimulus generalization training) was stable. Once 


\section{Condition 1}

Pretest

Phase A

Part One and Two of Initial Training
Phase B

Baseline

\section{Condition 2}

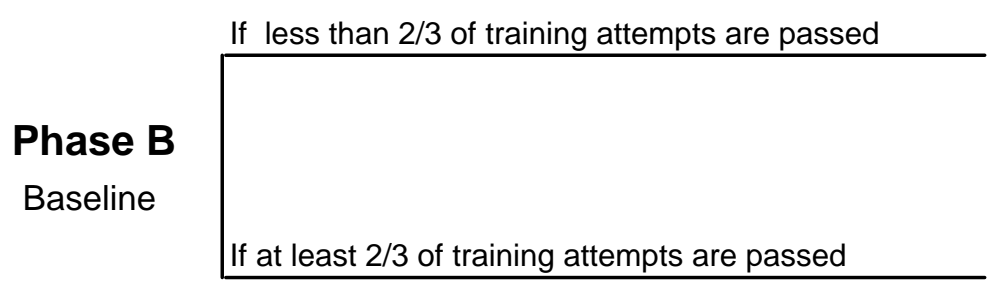

\section{Phase A}

Part Two of

Initial Training

\section{Phase C}

Transitive Stimulus

Generalization Training
Phase B

Baseline

\section{Condition 3}

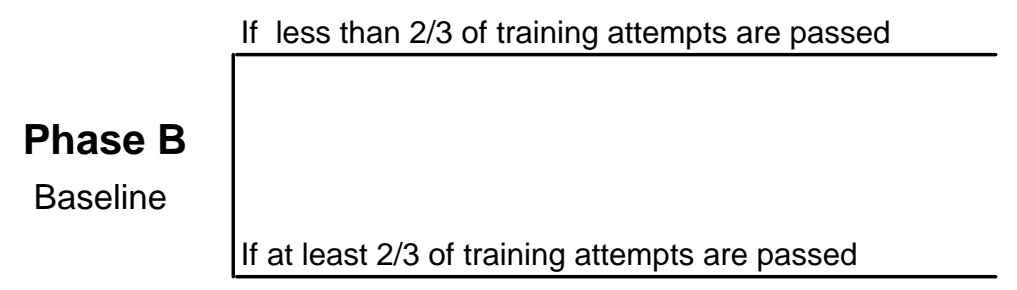

\section{Phase A}

Part Two of

Initial Training

\section{Phase D}

Within-Sets

Generalization Training
Phase B

Baseline

Figure 6. Data collection conditions. 
responding in Phase C was stable, consecutive sessions involved Condition 3 until responding in Phase D (within-sets generalization training) was stable.

Phase C stability criteria. Transitive stimulus generalization responding in Phase C was considered stable when 2/3 of transitive stimulus generalization attempts were passed in the last two blocks of Phase C. Within-sets generalization responding was considered stable when the greatest number of consecutive within-sets generalization attempts that were passed across the last two blocks of Phase $\mathrm{C}$ was greater than or equal to $r+3$, where $r$ was the greatest number of consecutive within-sets generalization attempts that were passed across the first three blocks of Phase C. For example, if r were to equal to four, then $r+3$ would equal seven, meaning that across the last two blocks of Phase C, the participant would have to pass seven or more consecutive within-sets generalization attempts. If the $\mathrm{r}+3$ criterion was not met after several sessions that involved Phase C, within-sets generalization responding was considered stable since within-sets generalization responding was shown consistently to not increase with increased transitive stimulus generalization training.

Phase D stability criteria. Responding in Phase D was considered stable when $2 / 3$ of the within-sets generalization attempts and $2 / 3$ of transitive stimulus generalization attempts were passed in the last two blocks of Phase D. Between-sets generalization responding was considered stable when the greatest number of consecutive between-sets generalization attempts that were passed across the last two blocks of Phase D was greater than or equal to $r+3$, where $r$ was the greatest number of consecutive between-sets generalization attempts that were passed across the first three blocks of Phase D. 
If the $\mathrm{r}+3$ between-sets generalization stability criterion that was used in Phase $\mathrm{D}$ could not be reached, it still was possible to determine whether or not within-sets generalization training had an effect on between-sets generalization based on the data pattern of previous sessions.

Reliability

Comparison selection. A trained observer was present for reliability checks for approximately $30 \%$ of the sessions (i.e., 8 sessions out a total of 27 sessions). During those sessions, accuracy of data recording on comparison selection during presentations of blocks of attempt cards was checked. The trained observer independently recorded data on the participant's selections of comparison stimuli on scorecards just as the researcher did.

Agreement on comparison selection was define as both observers (i.e., the researcher and a second trained observer) having marked an attempt as either passed or failed. Agreement on a session was determined by dividing the number of agreements by the number of agreements plus disagreements and multiplying the result by 100 . Agreement on comparison selection was 94\% or higher on each session.

Delivery of the positive consequence. An agreement check was calculated on the delivery of the positive consequence during (1) the transitive stimulus generalization training phases and (2) within-sets generalization training phases. Reliability was checked on $30 \%$ of the sessions that involved transitive stimulus generalization training or within-sets generalization training (i.e., 6 sessions out 20 sessions that involved generalization training). 
Agreement was defined as both observers recording that the researcher (1) did not indicate to the participant that the participant's response was correct or incorrect after an attempt at a test discrimination of the phase and (2) applied training to the training discriminations of phase. Agreement on delivery of the positive consequence was determined by dividing the number of agreements by the number of agreements plus disagreements and multiplying the result by 100 . Agreement on delivery of the positive consequence was $100 \%$ on each session. 
Chapter 4: Results

\section{Review of Research Questions}

The questions addressed in the current study are as follows. (1) Will increased training on transitive stimulus generalization increase the number of demonstrations of within-sets and between-sets generalization? (2) Will the addition of within-sets generalization training to transitive stimulus generalization training increase the number of demonstrations of between-sets generalization? In order to answer those questions, four typically developing five-year-old children were exposed to a pretest (start of baseline data), Phase A (initial training), Phase B (baseline), Phase C (transitive stimulus generalization training), and Phase D (the addition of within-sets generalization training to transitive stimulus generalization training).

The purpose of the study was to examine (1) whether or not within-sets generalization (WSG) and between-sets generalization (BSG) are dependent on transitive stimulus generalization (TSG), and (2) whether or not between-sets generalization (BSG) is dependent on within-sets generalization (WSG). A match-to-sample training procedure (Phase A), referred to as initial training, was used to train the four typically developing five-year-old children to select stimuli from set A (fractions in ratio form) in the presence of stimuli from either Set B or Set C (fractions in picture form). The emergence of TSG, WSG, and BSG then was examined (Phase B) through presentations of stimuli involved in initial training (see Figure 2 for stimuli involved in initial training) and stimuli not involved in initial training (see Figure 3 and Figure 4 for stimuli not involved in initial training). 
Participants then were given TSG training (Phase C) on the combinations of stimuli that were used to check for demonstrations of TSG. Demonstrations of TSG, WSG, and BSG were examined while the TSG training took place in order to examine whether TSG training increased demonstrations of WSG or BSG (the first research question).

While TSG training continued, participants were given WSG training (Phase D) on the certain combinations of stimuli that were used to check for demonstrations of WSG. Demonstrations of TSG, WSG, and BSG again were examined while the WSG training took place in order to examine whether the addition of WSG training to the TSG training increased demonstrations of BSG (the second research question).

In answer to the first research question, the data patterns of three of the four participants suggested that increased TSG training affected an increase in WSG responding. The data pattern of only one of the four participants offered limited support that increased TSG training affected an increase in BSG responding.

In answer the second research question, the data patterns of three of the four participants did not indicate that the addition of WSG training to TSG training affected an increase in BSG responding. The remaining data pattern suggested that the addition of WSG training to TSG training affected an increase in BSG responding.

\section{Review of Data Collection Conditions}

The first data collection condition (Condition 1) began with a pretest. See Figure 6 for the phases that were associated with each of the three data collection conditions. Participants were given a pretest to check the likelihood that the participant already had in his or her repertoire the discriminations that were to be trained and tested. Each of the 
four participants failed at least 2/3 of the attempt cards that were presented in the block of attempt cards used as a pretest in his or her first session of the study, which then was followed by Phase A (initial training) and Phase B (baseline).

Sessions that involved Condition 2 and Condition 3 always started with Phase B (baseline). If $2 / 3$ or more of the initial training attempt cards that were presented in that Phase B were passed, the next phase of the session became Phase C (TSG training) if in Condition 2 or the next phase became Phase D (the addition of WSG training) if in Condition 3. If less than $2 / 3$ of the initial training attempt cards in the baseline block were passed, the next phase of the session became Phase A (part two of initial training) if in Condition 2 or Condition 3. Following a return to part two of initial training, the final phase of the session was Phase B (baseline).

Review of Stability Criteria

Stability criteria were set so that in order to move past Condition 2, a participant first had to pass $2 / 3$ or more of the initial training attempt cards in the first Phase B of the session. That enabled him or her to start Phase C (TSG training). During Phase C, participants had to (1) pass $2 / 3$ or more of the TSG attempt cards presented in the last two blocks of Phase C and (2) meet the $\mathrm{r}+3$ WSG stability criterion. That is, in the last two blocks of Phase C, participants had to pass three more consecutive WSG attempt cards than $r$, the longest run of passed consecutive WSG attempt cards in the first three blocks of Phase C.

If the $\mathrm{r}+3$ WSG stability criterion was not met after several sessions that involved Condition 2, WSG responding was considered stable because either (1) WSG responding was shown consistently not to increase or decrease with increased TSG training or (2) the 
data pattern indicated that WSG responding had increased even though the $\mathrm{r}+3$ criterion was not met.

Stability criteria were set so that in order to complete Condition 3, a participant first had to pass $2 / 3$ or more of the initial training attempt cards in the first Phase $\mathrm{B}$ of the session. That enabled him or her to start Phase D (the addition of WSG training). During Phase D, participants had to (1) pass 2/3 or more of the TSG attempt cards and 2/3 or more of the WSG attempt cards presented in the last two blocks of Phase D and (2) meet the $\mathrm{r}+3$ BSG stability criterion. The $\mathrm{r}+3$ BSG stability criterion was the same as the $\mathrm{r}+3$ WSG stability criterion with the exception that BSG rather than WSG attempt cards were examined.

If the r+3 BSG stability criterion was not met after several sessions that involved Condition 3, BSG responding was considered stable because either (1) BSG responding was shown consistently not to increase or decrease with increased WSG training or (2) the data pattern indicated that BSG responding had increased even though the $\mathrm{r}+3$ criterion was not met.

\section{Data Collection Tables}

Each participant's data are represented in a table. Data collected from Participants 1, 2, 3, and 4 are shown in Tables 1, 2, 3, and 4, respectively. The tables show the number of passed attempt cards (i.e., TSG, WSG, BSG, and initial training) out of 6 that were presented in each block of a phase. Each B represents one block of attempt cards used as baseline (Phase B). Each C represents one block of attempt cards that received TSG training (Phase C). Each D represents one block of attempt cards that received WSG training in addition to TSG training (Phase D). 
Table 1

Correct Selections Made by Participant 1 in Each Phase, by Type of Attempt Card (TSG, WSG, BSG, and IT) and Session

\begin{tabular}{|c|c|c|c|c|c|c|c|}
\hline \multicolumn{2}{|r|}{ Phase } & $\begin{array}{c}\text { TSG } \\
\text { Passed }\end{array}$ & \multicolumn{2}{|c|}{ WSG } & \multicolumn{2}{|c|}{ BSG } & $\begin{array}{c}\text { IT } \\
\text { Passed }\end{array}$ \\
\hline & PT & 2 & 2 & & 3 & & 1 \\
\hline & A & & & & & & \\
\hline & B & 6 & 0 & & 4 & & 4 \\
\hline & $\mathrm{B}$ & 6 & 1 & & 3 & & 4 \\
\hline \multirow{18}{*}{ 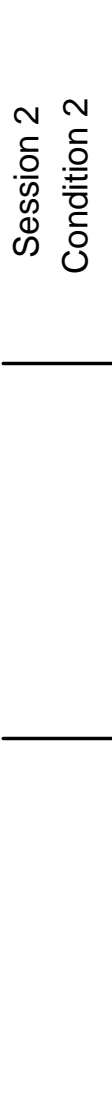 } & B & 5 & 1 & & 4 & & 6 \\
\hline & C & 5 & 2 & & 1 & & 6 \\
\hline & C & 5 & 3 & & 2 & & 5 \\
\hline & C & 6 & 3 & 3 & 3 & & 5 \\
\hline & C & 5 & 4 & & 2 & & 5 \\
\hline & C & $5^{\star}$ & 4 & 3 & 1 & & 6 \\
\hline & B & 6 & 4 & & 3 & & 5 \\
\hline & C & 5 & 4 & & 2 & & 6 \\
\hline & C & 6 & 4 & & 3 & & 6 \\
\hline & C & 6 & 5 & 4 & 3 & & 6 \\
\hline & C & 6 & 5 & & 4 & & 6 \\
\hline & C & $6^{*}$ & 5 & $8^{*}$ & 4 & & 6 \\
\hline & B & 6 & 4 & & 2 & & 6 \\
\hline & D & 6 & 5 & & 3 & & 6 \\
\hline & D & 6 & 5 & & 3 & & 6 \\
\hline & D & 6 & 5 & & 5 & 3 & 5 \\
\hline & D & 6 & 6 & & 5 & & 6 \\
\hline & D & $6^{\star}$ & $6^{*}$ & & 5 & $6^{*}$ & 6 \\
\hline
\end{tabular}

Note. TSG is transitive stimulus generalization, WSG is within-sets generalization, BSG is between-sets generalization, and IT is initial training. * means the value satisfied a stability criterion of the Condition. 
Table 2

Correct Selections Made by Participant 2 in Each Phase, by Type of Attempt Card (TSG, WSG, BSG, and IT) and Session

\begin{tabular}{|c|c|c|c|c|c|c|c|}
\hline \multicolumn{2}{|r|}{ Phase } & \multirow{2}{*}{$\begin{array}{c}\text { TSG } \\
\text { Passed } \\
1 \\
1\end{array}$} & \multicolumn{2}{|c|}{ WSG } & \multicolumn{2}{|l|}{ BSG } & $\begin{array}{c}\text { IT } \\
\text { Passed }\end{array}$ \\
\hline & $\mathrm{PT}$ & & 3 & & 3 & & 3 \\
\hline & A & & & & & & \\
\hline & $B$ & 3 & 3 & & 3 & & 1 \\
\hline & B & 4 & 3 & & 3 & & 1 \\
\hline & B & 4 & 0 & & 3 & & 3 \\
\hline & $A$ & & & & & & \\
\hline & B & 5 & 3 & & 0 & & 4 \\
\hline \multirow{6}{*}{ 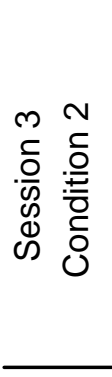 } & B & 3 & 1 & & 4 & & 4 \\
\hline & C & 2 & 2 & & 3 & & 5 \\
\hline & C & 4 & 2 & & 1 & & 5 \\
\hline & C & 5 & 3 & 3 & 2 & & 4 \\
\hline & C & 4 & 1 & & 2 & & 5 \\
\hline & C & $4^{*}$ & 4 & 4 & 3 & & 3 \\
\hline \multirow{6}{*}{ 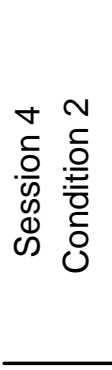 } & B & 4 & 3 & & 0 & & 4 \\
\hline & C & 5 & 3 & & 3 & & 4 \\
\hline & $\mathrm{C}$ & 3 & 4 & & 1 & & 3 \\
\hline & C & 4 & 2 & 2 & 2 & & 5 \\
\hline & C & 5 & 2 & & 3 & & 5 \\
\hline & C & $5^{*}$ & 1 & 1 & 2 & & 4 \\
\hline \multirow{6}{*}{ 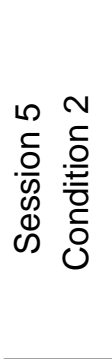 } & B & 6 & 2 & & 1 & & 4 \\
\hline & C & 6 & 2 & & 2 & & 3 \\
\hline & C & 5 & 3 & & 3 & & 4 \\
\hline & $\mathrm{C}$ & 3 & 3 & 2 & 1 & & 3 \\
\hline & C & 4 & 3 & & 1 & & 4 \\
\hline & C & $6^{*}$ & 2 & 3 & 2 & & 5 \\
\hline
\end{tabular}




\begin{tabular}{|c|c|c|c|c|c|c|}
\hline \multirow{6}{*}{ 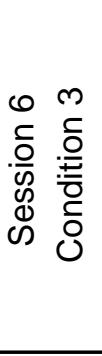 } & B & 5 & 3 & 2 & & 5 \\
\hline & D & 5 & 4 & 2 & \multirow{4}{*}{3} & 5 \\
\hline & D & 6 & 3 & 3 & & 6 \\
\hline & D & 5 & 5 & 3 & & 5 \\
\hline & D & 6 & 4 & 3 & & 5 \\
\hline & D & $6^{*}$ & $5^{\star}$ & 2 & 1 & 6 \\
\hline \multirow{6}{*}{ 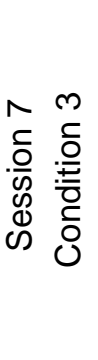 } & B & 4 & 4 & 3 & \multirow{5}{*}{1} & 4 \\
\hline & D & 6 & 4 & 1 & & 6 \\
\hline & D & 6 & 5 & 2 & & 4 \\
\hline & D & 5 & 6 & 1 & & 3 \\
\hline & D & 5 & 5 & 2 & & 5 \\
\hline & D & $6^{*}$ & $4^{*}$ & 2 & 2 & 5 \\
\hline
\end{tabular}

Note. TSG is transitive stimulus generalization, WSG is within-sets generalization, BSG is between-sets generalization, and IT is initial training. * means the value satisfied a stability criterion of the Condition. 
Table 3

Correct Selections Made by Participant 3 in Each Phase, by Type of Attempt Card (TSG, WSG, BSG, and IT) and Session

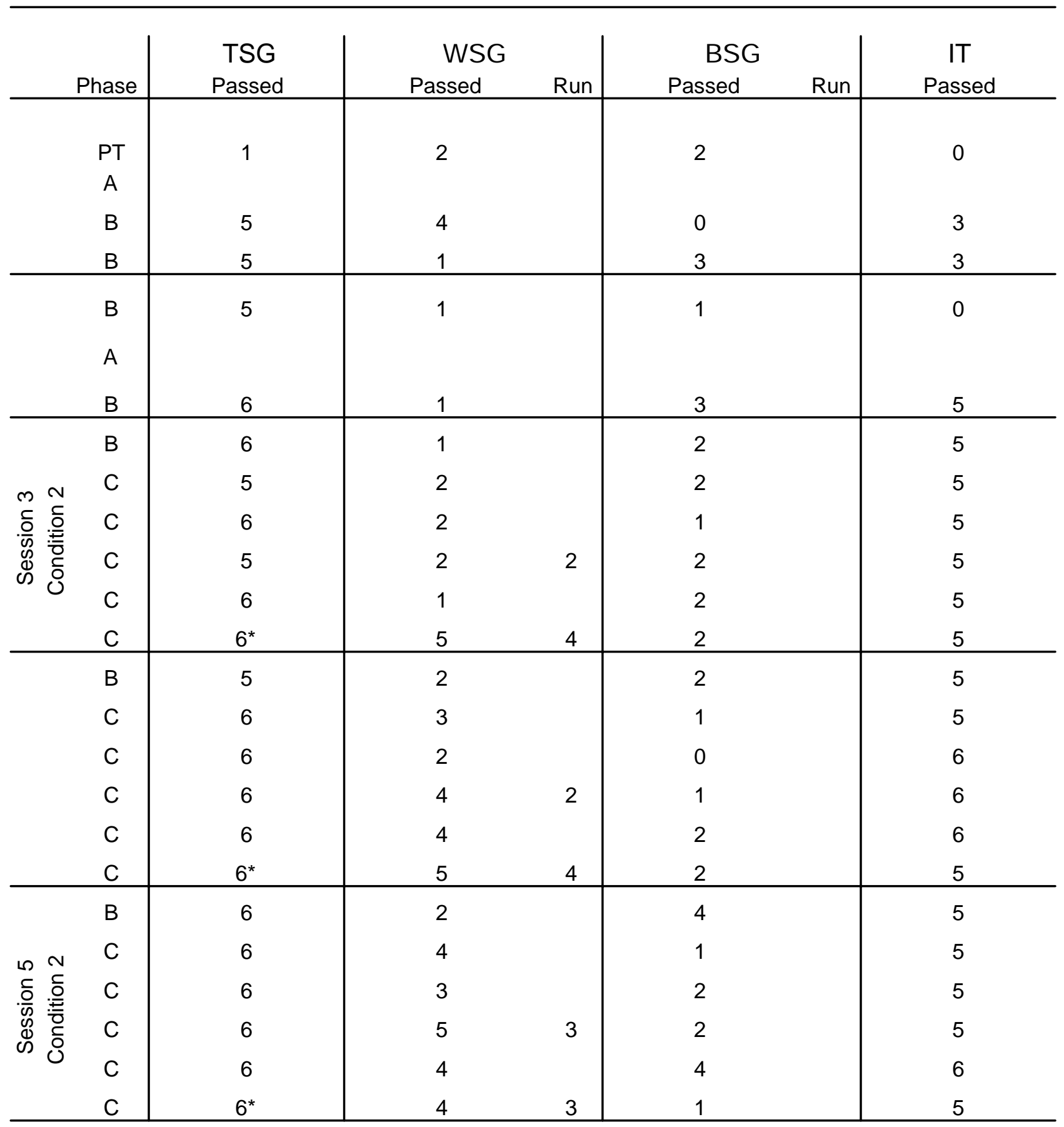




\begin{tabular}{|c|c|c|c|c|c|c|c|}
\hline \multirow{6}{*}{ 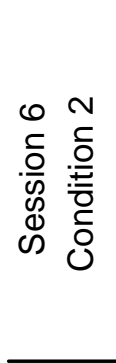 } & B & 6 & 3 & & 3 & & 5 \\
\hline & C & 6 & 5 & \multirow{4}{*}{3} & 2 & & 6 \\
\hline & C & 6 & 3 & & 1 & & 6 \\
\hline & C & 6 & 4 & & 2 & & 6 \\
\hline & C & 6 & 5 & & 4 & & 5 \\
\hline & C & $6^{*}$ & 5 & 4 & 1 & & 6 \\
\hline \multirow{6}{*}{ 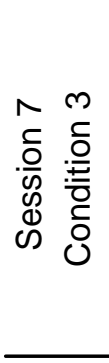 } & B & 6 & 3 & & 2 & \multirow{5}{*}{2} & 5 \\
\hline & D & 6 & 5 & & 2 & & 6 \\
\hline & D & 6 & 6 & & 4 & & 5 \\
\hline & D & 6 & 6 & & 2 & & 5 \\
\hline & D & 6 & 5 & & 4 & & 5 \\
\hline & D & $6^{*}$ & $6^{\star}$ & & 3 & 3 & 6 \\
\hline \multirow{6}{*}{ 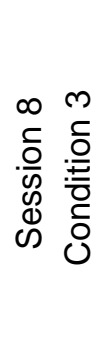 } & B & 6 & 6 & & 2 & \multirow{5}{*}{3} & 6 \\
\hline & D & 6 & 6 & & 3 & & 6 \\
\hline & D & 6 & 5 & & 4 & & 5 \\
\hline & D & 6 & 5 & & 1 & & 6 \\
\hline & D & 6 & 6 & & 2 & & 5 \\
\hline & D & $6^{*}$ & $6^{\star}$ & & 4 & 3 & 5 \\
\hline
\end{tabular}

Note. TSG is transitive stimulus generalization, WSG is within-sets generalization, BSG is between-sets generalization, and IT is initial training. * means the value satisfied a stability criterion of the Condition. 
Table 4

Correct Selections Made by Participant 4 in Each Phase, by Type of Attempt Card (TSG, WSG, BSG, and IT) and Session

\begin{tabular}{|c|c|c|c|c|c|c|c|}
\hline \multicolumn{2}{|r|}{ Phase } & \multirow{2}{*}{$\begin{array}{c}\begin{array}{c}\text { TSG } \\
\text { Passed }\end{array} \\
0\end{array}$} & \multicolumn{2}{|c|}{ WSG } & \multicolumn{2}{|c|}{ BSG } & $\begin{array}{c}\text { IT } \\
\text { Passed }\end{array}$ \\
\hline & PT & & 2 & & 0 & & 0 \\
\hline & A & & & & & & \\
\hline & B & 4 & 1 & & 0 & & 5 \\
\hline & B & 4 & 1 & & 0 & & 5 \\
\hline & B & 4 & 2 & & 1 & & 2 \\
\hline & A & & & & & & \\
\hline & B & 5 & 2 & & 2 & & 5 \\
\hline \multirow{12}{*}{ 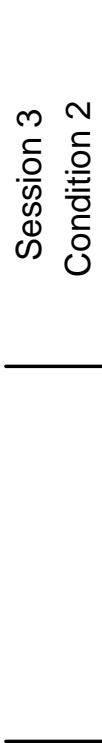 } & B & 5 & 2 & & 5 & & 5 \\
\hline & C & 5 & 5 & & 2 & & 5 \\
\hline & C & 5 & 1 & & 1 & & 5 \\
\hline & $\mathrm{C}$ & 5 & 1 & 5 & 0 & & 6 \\
\hline & $\mathrm{C}$ & 5 & 3 & & 0 & & 6 \\
\hline & C & $5^{\star}$ & 3 & 4 & 2 & & 5 \\
\hline & $B$ & 5 & 3 & & 0 & & 4 \\
\hline & C & 6 & 4 & & 2 & & 5 \\
\hline & $\mathrm{C}$ & 6 & 3 & & 4 & & 5 \\
\hline & $\mathrm{C}$ & 6 & 4 & 3 & 2 & & 5 \\
\hline & $\mathrm{C}$ & 6 & 4 & & 1 & & 5 \\
\hline & $\mathrm{C}$ & $6^{*}$ & 4 & 5 & 2 & & 6 \\
\hline \multirow{6}{*}{ 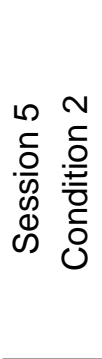 } & B & 5 & 3 & & 1 & & 5 \\
\hline & C & 6 & 3 & & 2 & & 6 \\
\hline & C & 6 & 4 & & 2 & & 6 \\
\hline & C & 6 & 4 & 4 & 3 & & 6 \\
\hline & C & 6 & 5 & & 2 & & 6 \\
\hline & C & $6^{*}$ & 5 & 6 & 0 & & 6 \\
\hline
\end{tabular}




\begin{tabular}{|c|c|c|c|c|c|c|c|}
\hline \multirow{6}{*}{ 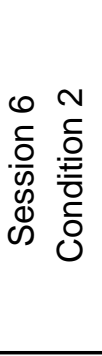 } & B & 6 & 3 & & 3 & & 4 \\
\hline & C & 6 & 3 & & 3 & & 5 \\
\hline & C & 6 & 5 & & 1 & & 5 \\
\hline & C & 6 & 4 & 3 & 2 & & 5 \\
\hline & C & 6 & 5 & & 2 & & 6 \\
\hline & C & $6^{\star}$ & 4 & 4 & 2 & & 6 \\
\hline \multirow{6}{*}{ 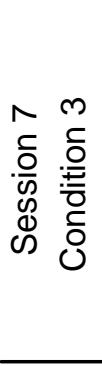 } & B & 6 & 3 & & 3 & & 4 \\
\hline & D & 6 & 5 & & 3 & & 5 \\
\hline & D & 6 & 5 & & 4 & & 5 \\
\hline & D & 6 & 6 & & 3 & 2 & 6 \\
\hline & D & 6 & 6 & & 4 & & 6 \\
\hline & D & $6^{*}$ & $6^{*}$ & & 4 & 3 & 6 \\
\hline \multirow{6}{*}{ 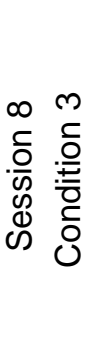 } & B & 6 & 6 & & 4 & & 5 \\
\hline & D & 6 & 6 & & 3 & & 6 \\
\hline & D & 6 & 6 & & 4 & & 6 \\
\hline & D & 6 & 6 & & 3 & 3 & 5 \\
\hline & D & 6 & 6 & & 4 & & 6 \\
\hline & D & $6^{\star}$ & $6^{*}$ & & 5 & $7^{*}$ & 6 \\
\hline
\end{tabular}

Note. TSG is transitive stimulus generalization, WSG is within-sets generalization, BSG is between-sets generalization, and IT is initial training. * means the value satisfied a stability criterion of the Condition. 
Representations of passed attempt cards are not shown in the tables for Phase A. That is because Phase A was initial training and did not involve presentations of blocks of attempt cards. Phase A was included in the data collection tables to indicate in which sessions initial training took place.

The phases involved in the data collection conditions of each session are shown in the tables. For example, in Table 1 next to Session 2 is Condition 2, meaning that Session 2 involved Condition 2. Next to Session 2 and Condition 2 are Phase B (i.e., baseline) and Phase C (i.e., TSG training), meaning that Phase B and Phase C were involved in the Condition 2 that took place in Session 2 for Participant 1 (Table 1).

The amount of passed attempt cards in each phase is shown in the tables by type of attempt card (e.g., TSG). The Passed column contains the number of passed attempt cards out of 6 that were presented in each block of a phase. An asterisk next to the number of passed TSG attempt cards shown in the last block of a set of Phase C blocks indicates that in the last two blocks of the set, 2/3 or more of the TSG cards were passed (i.e., the Condition 2 and Condition 3 TSG stability criterion was met). An asterisk next to the number of passed WSG attempt cards shown in the last block of a set of Phase D blocks indicates that in the last two blocks of the set, 2/3 or more of the WSG cards were passed (i.e., the Condition 3 WSG stability criterion was met).

For example, Table 1 shows that in Session 2, Participant 1 passed 5 of the 6 TSG attempt cards that were presented in both the fourth and fifth blocks of the set of Phase C blocks. Thus, of the 12 TSG attempt cards that were presented in the last two blocks of the set of Phase C blocks more than 2/3 were passed (i.e., 10 of the 12 TSG attempt cards were passed). 
The Run column shown under WSG contains the greatest number of consecutive passed attempt cards that were presented in (1) the first three blocks of each set of Phase C blocks prior to the start of Phase D and (2) the last two blocks of each set of Phase C prior to the start of Phase D. The Run column shown under BSG contains the greatest number of consecutive passed attempt cards that were presented in (1) the first three blocks of each set of Phase D blocks and (2) the last two blocks of each set of Phase D.

The run value next to the third block of a set of Phase $\mathrm{C}$ or Phase D blocks is the r-value in the $r+3$ WSG stability criterion and the $r+3$ BSG stability criterion. The run value next to the fifth block of a set of Phase $\mathrm{C}$ or Phase $\mathrm{D}$ blocks is the value to which the $r+3$ value was compared in order to evaluate whether the $r+3$ WSG or $r+3$ BSG stability criterion was met. For example, under WSG on Table 1 in Session 3, the run value of 4 is shown next to the third Phase $\mathrm{C}$ block, meaning that in the first three blocks of that set of Phase C blocks, there were 4 consecutively passed WSG attempt cards. Thus, the r-value was 4 for that set of Phase $C$ blocks and $r+3$ was 7 . The run value of 8 is shown next to the fifth Phase C block, meaning that in the last two blocks of that set there were 8 consecutively passed WSG attempt cards. An asterisk is shown next to the 8 to indicate that the $\mathrm{r}+3$ WSG stability criterion was met (i.e., there were more than $\mathrm{r}+3$ consecutively passed WSG attempt cards in the last two blocks of the set).

\section{Data Pattern Figures}

The data patterns of WSG and BSG responding for Participants 1, 2, 3, and 4 are shown in Figures 7, 8, 9, and 10, respectively. Each data point on the figures represents the number of passed WSG or BSG attempt cards out of 6 that were presented in a block 

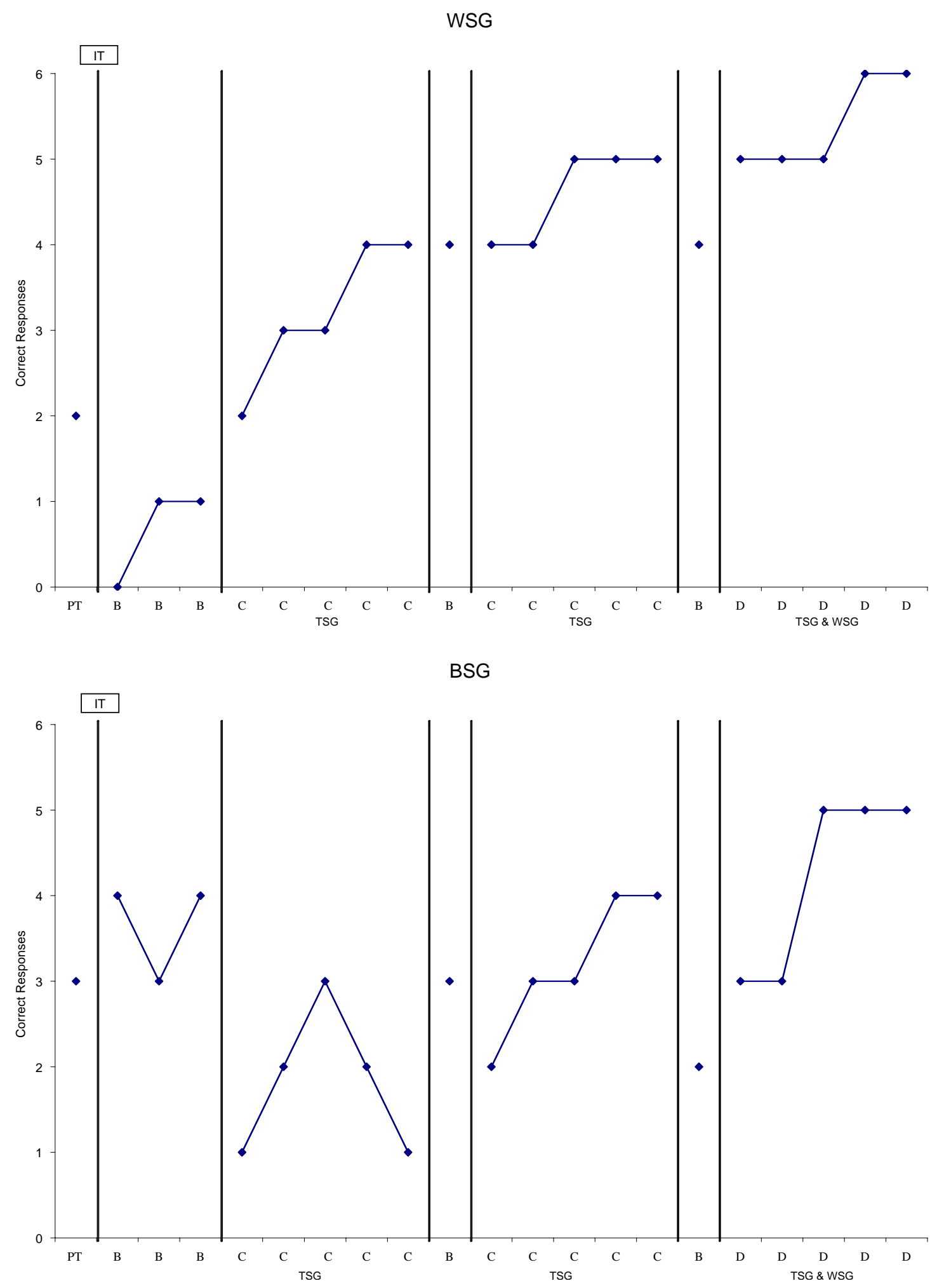

Figure 7. WSG and BSG data pattern of Participant 1. 

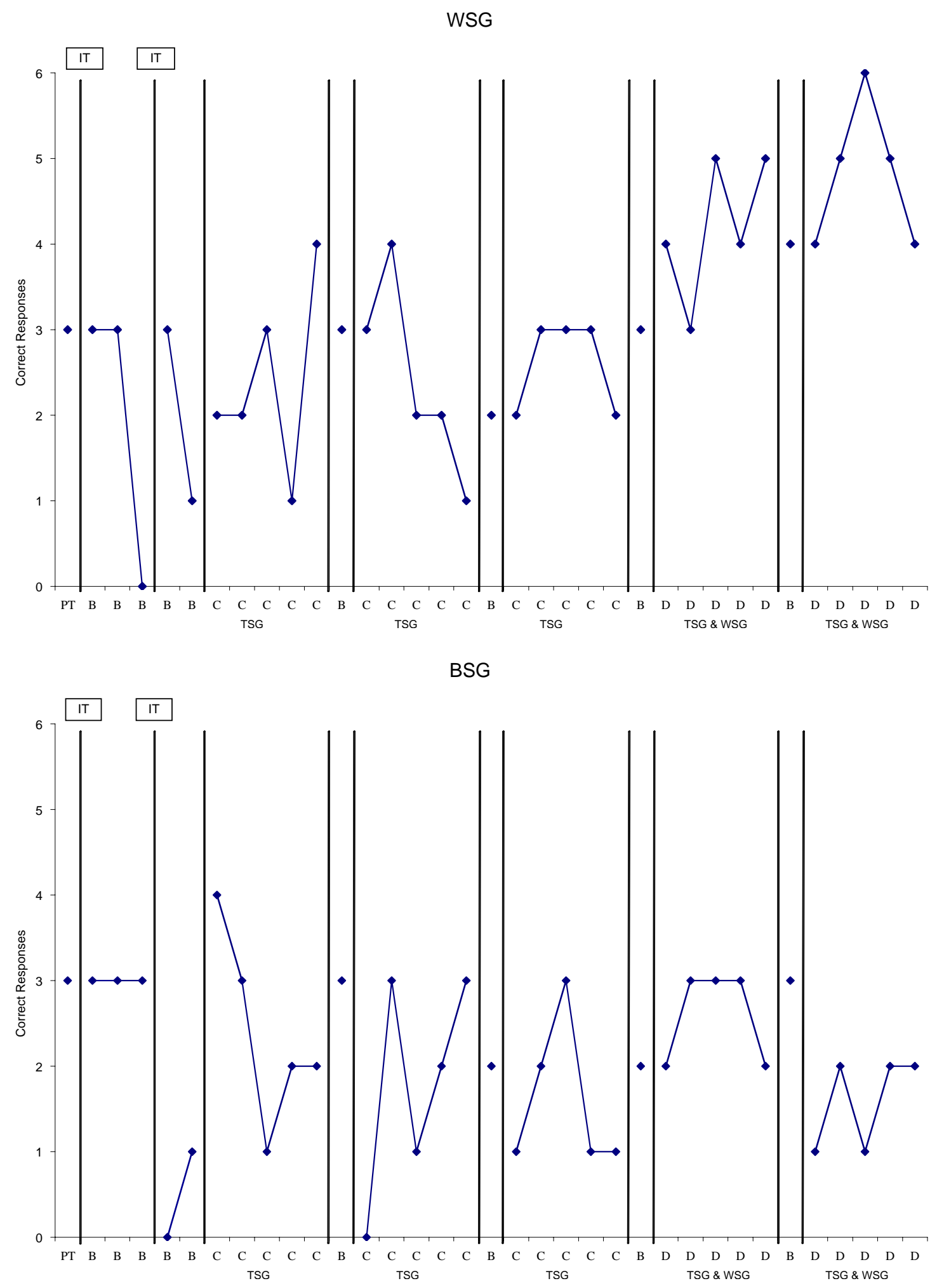

Figure 8. WSG and BSG data pattern of Participant 2. 

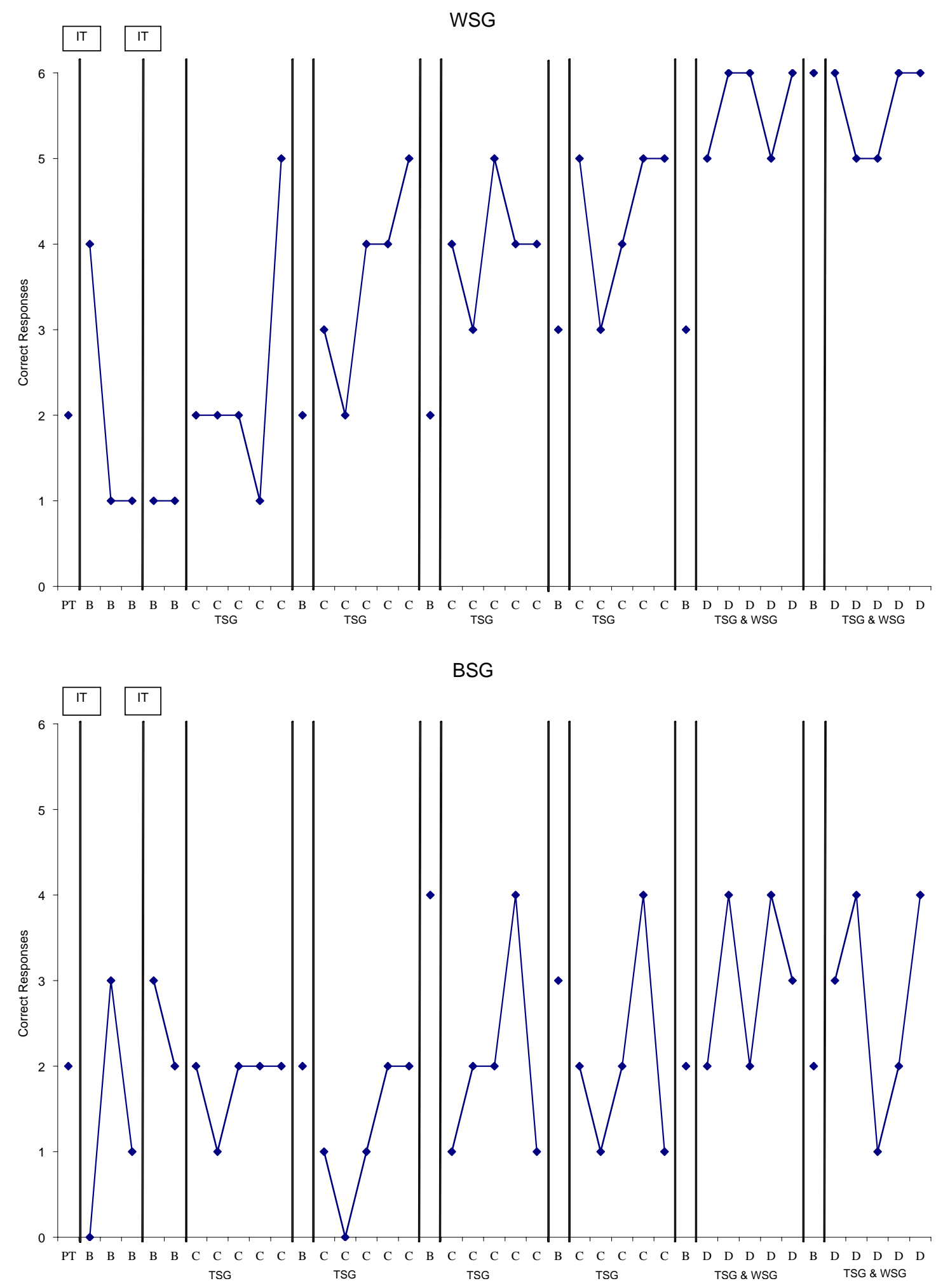

Figure 9. WSG and BSG data pattern of Participant 3. 

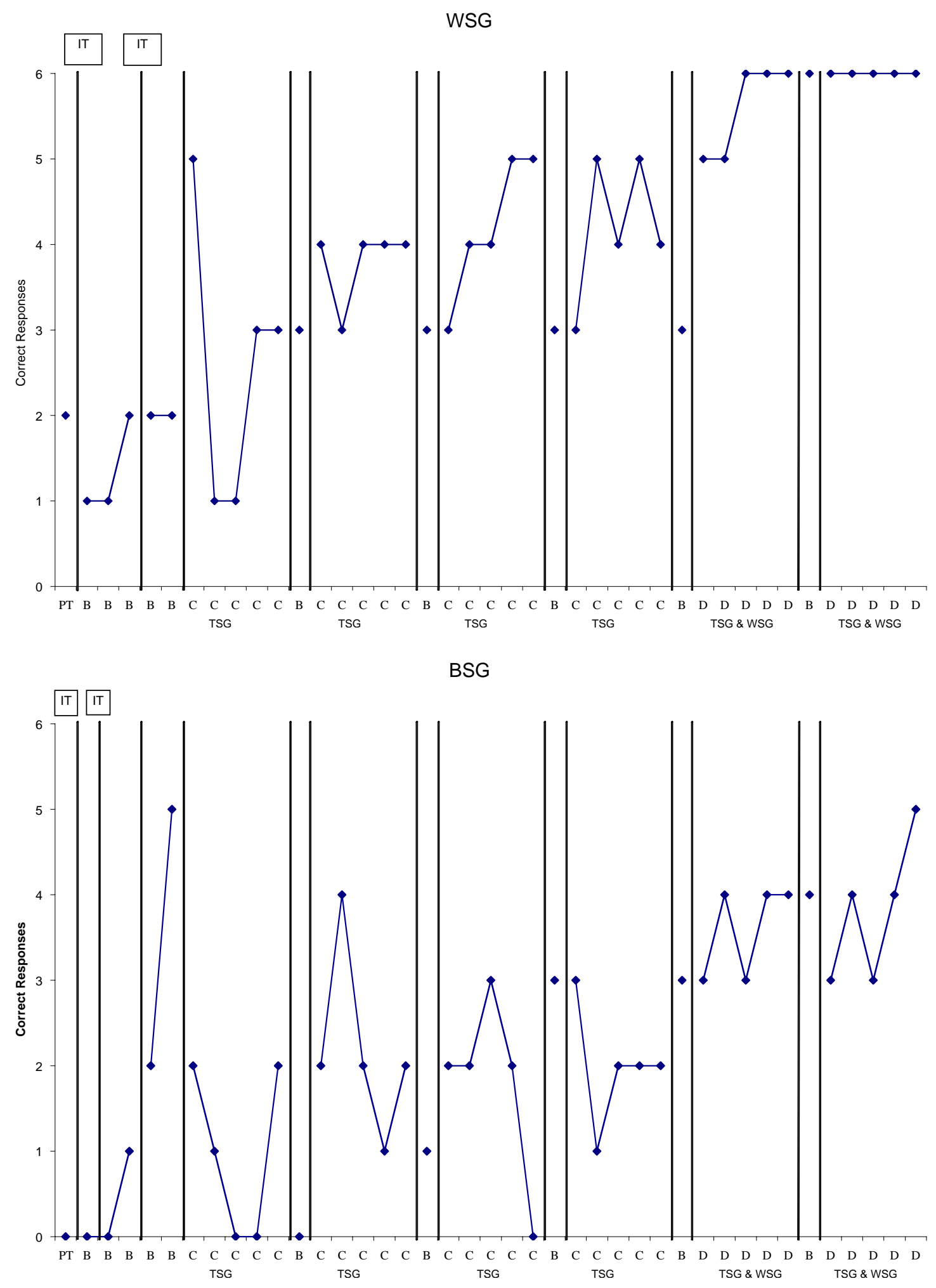

Figure 10. WSG and BSG data pattern of Participant 4. 
of attempt cards. That is, the value of each data point is shown in a Passed column on the tables.

Phase lines (i.e., the vertical lines in the figures) were used to indicate phase changes. Lines that are labeled IT indicate where initial training took place. Blocks of attempt cards are labeled along the x-axis of each graph. PT stands for pretest, each B stands for one block of attempt cards used as baseline (Phase B), each C stands for one block of attempt cards that received TSG training (Phase C), and each D stands for one block of attempt cards that received WSG training and TSG training (Phase D).

\section{Stability in Sessions}

The data collection tables show that participants satisfied the stability criteria of Condition 2 (sessions involving Phase C) and Condition 3 (sessions involving Phase D) at different points. Hence, the number of sessions that were needed by each participant varied. Participant 1 had a total of four sessions, Participant 2 had a total of seven sessions, Participant 3 had a total of eight sessions, and Participant 4 had a total of eight sessions. The way in which each participant met the stability criteria of Condition 2 and Condition 3 are described in the following paragraphs.

Participant 1. Participant 1 started Phase C (TSG training) during Session 2 because $2 / 3$ or more of the initial training attempt cards presented in the Phase B block (baseline) of Session 2 were passed. In Sessions 2, 3, and 4, TSG responding met the Condition 2 and Condition 3 TSG stability criterion of correct responses to 2/3 or more of the TSG attempt cards presented in the last two blocks of the session. In Session 4, the WSG stability criterion of correct responses to 2/3 or more of the WSG attempt cards presented in the last two blocks of the session was met. 
Participant 1 remained in Condition 2 after Session 2 because the $r+3$ WSG stability criterion was not met. In Session 3, the r+3 WSG stability criterion was met, meaning that Participant 1 moved to Condition 3 for Session 4. In Session 4 the r+3 BSG stability criterion was met, meaning that participation was complete at the end of Session 4 since (1) the r+3 BSG stability criterion had been met and (2) the TSG and WSG responding criteria also had been met. See Table 1 for Participant 1 results. An asterisk is shown next to values that satisfied a stability criterion that was discussed in the previous paragraphs.

Participant 2. Participant 2 did not start Phase C (TSG training) in Session 2 because less than $2 / 3$ of the initial training attempt cards presented in the first Phase $B$ block (baseline) of Session 2 were passed. Phase C was started in Session 3 because 2/3 or more of the initial training attempt cards presented in the Phase B block (baseline) of Session 3 were passed. In Sessions 3, 4, 5, 6 and 7, TSG responding met the Condition 2 and Condition 3 TSG stability criterion of correct responses to 2/3 or more of the TSG attempt cards presented in the last two blocks of the session. In Sessions 6 and 7 the WSG stability criterion of correct responses to $2 / 3$ or more of the WSG attempt cards presented in the last two blocks of the session was met.

Sessions 3, 4, and 5 for Participant 2 involved Condition 2 with Phase C. Although the r+3 WSG stability criterion had not been met, Participant 2 moved to Condition 3 after Session 5 since (1) WSG responding was not shown to consistently increase or decrease with increased exposure to training and (2) the TSG responding criterion had been met. 
Sessions 6 and 7 for Participant 2 involved Condition 3 with Phase D. The $\mathrm{r}+3$ BSG stability criterion was not met in either Session 6 or Session 7. Participation for Participant 2 was considered complete after Session 7 since (1) BSG responding was not shown to consistently increase or decrease with increased exposure to training and (2) the TSG and WSG responding criteria had been met. See Table 2 for Participant 2 results. An asterisk is shown next to values that satisfied a stability criterion that was discussed in the previous paragraphs.

Participant 3. Participant 3 did not start Phase C (TSG training) in Session 2 because less than $2 / 3$ of the initial training attempt cards presented in the Phase B block (baseline) of Session 2 were passed. Phase C was started in Session 3 because 2/3 or more of the initial training attempt cards presented in the first Phase B block (baseline) of Session 3 were passed. In Sessions 3, 4, 5, 6, 7 and 8, TSG responding met the Condition 2 and Condition 3 TSG stability criterion of correct responses to 2/3 or more of the TSG attempt cards presented in the last two blocks of the session. In both Sessions 7 and 8, the WSG stability criterion of correct responses to 2/3 or more of the WSG attempt cards presented in the last two blocks of a session was met.

Sessions 3, 4, 5 and 6 for Participant 3 involved Condition 2 with Phase C. Although the $\mathrm{r}+3$ WSG stability criterion had not been met, Participant 3 moved to Condition 3 after Session 6 since (1) the number of passed WSG attempt cards presented in the Phase C blocks gradually increased and (2) the TSG responding criterion had been met.

Sessions 7 and 8 for Participant 3 involved Condition 3 with Phase D. The $\mathrm{r}+3$ BSG stability criterion was not met in either Session 7 or Session 8. Participation for 
Participant 3 was considered complete after Session 8 since (1) BSG responding was not shown to consistently increase or decrease with increased exposure to the training procedures and (2) the TSG and WSG responding criteria had been met. See Table 3 for Participant 3 results. An asterisk is shown next to values that satisfied a stability criterion that was discussed in the previous paragraphs.

Participant 4. Participant 4 did not start Phase C (TSG training) in Session 2 because less than $2 / 3$ of the initial training attempt cards presented in the Phase B block (baseline) of Session 2 were passed. Phase C was started in Session 3 because 2/3 or more of the initial training attempt cards presented in the first Phase B block (baseline) of Session 3 were passed. In Sessions 3, 4, 5, 6, 7 and 8, TSG responding met the Condition 2 and Condition 3 TSG stability criterion of correct responses to 2/3 or more of the TSG attempt cards presented in the last two blocks of the session. In both Sessions 7 and 8, the WSG stability criterion of correct responses to 2/3 or more of the WSG attempt cards presented in the last two blocks of a session was met.

Sessions 3, 4, 5 and 6 for Participant 4 involved Condition 2 with Phase C. Although the r+3 WSG stability criterion had not been met, Participant 4 moved to Condition 3 after Session 6 since (1) the number of passed WSG attempt cards presented in the Phase C blocks gradually increased and (2) the TSG responding criterion had been met.

Sessions 7 and 8 for Participant 4 involved Condition 3 with Phase D. Participation for Participant 4 was considered complete after Session 8 since (1) the $r+3$ BSG stability criterion was met in Session 8 and (2) the TSG and WSG responding 
criteria had been met. See Table 4 for Participant 4 results. An asterisk is shown next to values that satisfied a stability criterion that was discussed in the previous paragraph. Function of the Stability Criteria

Stability criteria were used for the purpose of determining (1) which phases would be involved in Condition 2 and Condition 3, (2) when each participant moved from Condition 2 to Condition 3, and (3) when each participant had completed his or her participation. When a participant's responding met a stability criterion (e.g., the $\mathrm{r}+3$ WSG stability criterion) it suggested that the procedures used had affected an increase in responding to a certain type of attempt card. However, determinations as to which aspects of the procedures had affected responding could not be made just from examining when the stability criteria were met. For instance, it could not be determined if increases in responding to a certain type of attempt card (1) were affected simply through increased exposure to the attempt cards (i.e., a general training effect) or (2) were functionally related to certain types of generalization training.

In order to determine which aspects of the training procedures affected the changes in WSG and BSG responding, the data pattern of each participant was examined in terms of (1) a TSG training effect on WSG responding and BSG responding and (2) a WSG training effect on BSG responding. The first research question (i.e., will increased training on TSG increase the number of demonstrations of WSG and BSG?) is addressed through the examination of a TSG training effect. The second research question (i.e., will the addition of WSG training to TSG training increase the number of demonstrations of BSG?) is addressed through the examination of a WSG training effect. 
Data were examined through visual inspection of changes that occurred in the data pattern of each participant. Changes in the data were evaluated based on magnitude (i.e., changes in mean and level of the data) and rate (i.e., trend and latency) of the change.

Participant 1 Data Pattern

Participant 1 WSG responding. WSG responding did increase across sets of Phase C and Phase D. WSG responding showed an upward trend across each set of Phase C blocks (i.e., blocks of TSG training), and the set of Phase D blocks (i.e., blocks of WSG and TSG training). Along with the upward trends (i.e., trend lines with a positive slopes), the mean value of WSG responding across each set of Phase C blocks and the set of Phase D blocks increased.

The data pattern also shows that as WSG responding increased, it became more stable. That is, the range between the highest data point and the lowest data point decreased from a range of 2 in the first set of Phase $C$ blocks to a range of 1 in the second set of Phase C blocks.

It appears that the increase in WSG responding was due to TSG training rather than just increased exposure to the attempt cards. For instance, when TSG was introduced in the first Phase C block there was an upward shift in the level of the data pattern, which suggests that the introduction of TSG training affected an increase in WSG responding.

In addition to that increase in WSG responding after the introduction of TSG training, responding did not continue to increase from the end of the first set of Phase C blocks to the beginning of the second set of Phase C blocks (i.e., responding did not change when TSG training was removed). After a latency period of two blocks, 
responding showed an increase in the second set of Phase $\mathrm{C}$ blocks. In the Phase B block that followed the second set of Phase C blocks, responding decreased to the previous Phase B level, and responding increased with the reintroduction of training in the set of Phase D blocks. As shown in the data pattern, there was an upward shift in the level of WSG responding from the Phase B to the first Phase C block (i.e., the introduction of TSG training) and then again to the first Phase D block (i.e., the introduction of WSG training and TSG training). That pattern suggests that the increase in WSG responding was due to training (i.e., responding either decreased or stayed the same when training was removed); however, since Phase D was the addition of WSG training to TSG training, it is not clear if the continued increase in responding from the last set of Phase C blocks to the set of Phase D blocks was due to increased TSG training or to the addition of WSG training.

In summary, for Participant 1 WSG responding, the data pattern supports a training effect on WSG responding. The pattern also suggests that the presence of TSG training affected an increase in WSG responding.

Participant 1 BSG responding. BSG responding eventually did increase across the last set of Phase $\mathrm{C}$ and the Phase D block. A systematic change in the data pattern did not appear until the second application of TSG training (i.e., the second set of Phase C blocks). In that second set of Phase C blocks, responding showed an upward trend. Responding decreased once training was removed in the Phase B (baseline) that followed, and there was a shift in level from Phase B to the first block of the set of Phase D blocks. Responding again showed an upward trend across the Phase D blocks. In 
addition, the mean value of responding increased from the last set of Phase $\mathrm{C}$ blocks to the set of Phase D blocks.

The pattern of an upward trend across blocks of training (i.e., the second set of Phase C blocks) followed by a decrease in responding in baseline, followed by and upward shift in level and upward trend across blocks of training (i.e., the set of Phase D blocks) indicated that training did affect an increase in BSG responding. The upward trend across the second set of Phase $\mathrm{C}$ blocks followed by the decrease in responding offers some limited support that TSG training affected an increase in BSG responding. Since Phase D was the addition of WSG training to TSG training, it is not clear if the increase in responding from the last set of Phase C blocks to the set of Phase D blocks was due to increased TSG training or to the addition of WSG training.

In summary, for Participant 1 BSG responding, the data pattern supports a training effect on BSG responding. The pattern also offers limited support that TSG training affected an increase in BSG responding.

\section{Participant 2 Data Pattern}

Participant 2 WSG responding. Systematic changes in WSG responding were not seen prior to the sets of Phase D blocks (i.e., addition of WSG training to TSG training). The patterns of responding shown in sets of Phase B blocks (i.e., baseline) prior to the start of TSG training were similar to the patterns of WSG responding in each set of Phase C blocks (i.e., blocks of TSG training).

Although the range between the highest data point and the lowest data point of each set of Phase C blocks decreased from a range of 3 in both the first and second set to range of 1 in the third set (a suggestion that TSG responding may have served to stabilize 
WSG responding), the data pattern does not indicate that TSG training affected an increase in WSG responding. Table 2 shows that, responding to initial training attempt cards frequently dropped below 4 correct responses in the Phase C blocks, implying that motivational factors frequently may have affected the poor WSG responding of Participant 2 across sets of Phase C blocks.

There was an increase in the mean value of WSG responding from the sets of Phase C blocks to the first set of Phase D blocks. Although there continued to be a great deal of variability in WSG responding across the sets of Phase D blocks, there appears to have been an increase in WSG responding across the sets of Phase D blocks. That is, there was a notable increase in the mean value of responding from the sets of Phase C blocks to the sets of Phase D blocks, and there was an upward trend across the first set of Phase D blocks.

Since Phase D was the addition of WSG training to TSG training, it is not clear which part of training (TSG training or WSG training) affected that change. It is likely, though, that WSG training affected the increase since previous TSG training did not affect a change in WSG responding, and WSG training was direct training on the WSG cards presented in each block.

In summary, for Participant 2 WSG responding, the data pattern does suggest a training effect on WSG responding. The pattern does not indicate that TSG training affected the increase in WSG responding.

Participant 2 BSG responding. Systematic changes in BSG responding were not seen in the data pattern. The range between the highest data point and the lowest data point of BSG responding in sets of Phase B blocks prior to the start of TSG training was 
similar to the range between the highest and lowest points of BSG responding in the sets of Phase C blocks (blocks of TSG training) and Phase D blocks (blocks of WSG and TSG training).

As discussed previously, motivational factors frequently may have affected the poor BSG responding of Participant 2, which was evidenced by the drops in responses to the initial training attempt cards to below 4 correct responses in the Phase $\mathrm{C}$ blocks and Phase D blocks.

In summary, for Participant 2 BSG responding, the data pattern does not support a training effect on BSG responding. Hence, TSG training was not shown to affect an increase in BSG responding, and WSG training was not shown to affect an increase in BSG responding.

Participant 3 Data Pattern

Participant 3 WSG responding. WSG responding did increase across the sets of Phase C and Phase D blocks. There was an upward trend in WSG responding in each set of Phase C and Phase D blocks, and the mean value of WSG responding increased across the sets of Phase $\mathrm{C}$ and Phase D blocks. That data pattern indicates that WSG responding increased across the sets of Phase C and Phase D blocks.

It appears that the increase in WSG responding was due to increased TSG training rather than just increased exposure to the attempt cards. For instance, in the last four Phase B blocks (baseline) prior to the start of TSG training in the Phase C blocks, WSG responding was stable at a value of 1 correct WSG response. When TSG training was introduced in the first Phase C block, there was an upward shift in level of the data 
pattern, which suggests that the introduction of TSG training affected an increase in WSG responding.

The level changes in the data pattern at the beginning and end of each set of Phase C blocks (i.e., blocks of TSG training) offer further evidence that TSG training affected the increase in WSG responding. The downward shifts in the level from the last block of each set of Phase C blocks to the Phase B block (baseline) that followed were to values that were below the mean value of WSG responding in the preceding set of Phase C blocks. Those downward shifts indicate that when TSG training was withheld during the Phase B blocks, WSG responding decreased.

The upward shift in level from each Phase B block to the first block of the set of Phase $\mathrm{C}$ blocks that followed was to a value greater than the mean value of the previous set of Phase C blocks. Those upward shifts indicate that when TSG training was reintroduced in the first block of each set of Phase C blocks, WSG increased. Upward shifts in level from Phase B to Phase $\mathrm{C}$ following the downward shifts from Phase $\mathrm{C}$ to Phase B indicate that responding did not slowly increase with the reintroduction of TSG training.

Just as before when training was reintroduced following a Phase B block, there was an upward shift in level from the Phase B block to the first Phase D block (i.e., the addition of WSG training to TSG training). In addition, there was less variability in the WSG responding in the sets of Phase D blocks compared to the Phase C blocks, which suggests that the addition of WSG training may have served to increase stability. Since WSG responding had been shown to increase across previous blocks of TSG training (i.e., Phase C), it is not clear whether or not the continued increase in WSG responding 
from the last set of Phase C blocks to the first set of Phase D blocks was due to increased exposure to TSG training or the addition of WSG training.

In summary, for Participant 3 WSG responding, the data pattern supports a training effect on WSG responding. The pattern also indicates that TSG training affected an increase in WSG responding.

Participant 3 BSG responding. Systematic changes in BSG responding were not seen in the data pattern. The range between the highest data point and the lowest data point of BSG responding in sets of Phase B blocks prior to the start of TSG training was similar to the range between the highest and lowest points of BSG responding in the sets of Phase C blocks (blocks of TSG training) and Phase D blocks (blocks of WSG and TSG training).

The data pattern indicates that BSG responding did not become less variable across the sets of Phase $\mathrm{C}$ blocks and Phase D blocks. That is, the range between the highest and lowest points in each set of Phase C and Phase D blocks did not decrease with increase exposure to training.

In summary, for Participant 3 BSG responding, the data pattern does not support a training effect on BSG responding. Hence, TSG training was not shown to affect an increase in BSG responding, and WSG training was not shown to affect an increase in BSG responding.

\section{Participant 4 Data Pattern}

Participant 4 WSG responding. WSG responding did increase across the sets of Phase C and Phase D blocks. With the exception of the first set of Phase C blocks and last set of Phase D blocks, there was an upward trend in WSG responding in each set of 
Phase C and Phase D blocks, and there was a gradual increase in the mean value of WSG responding across the sets of Phase $\mathrm{C}$ and Phase $\mathrm{D}$ blocks. In the last set of Phase $\mathrm{D}$ blocks, there was zero trend due to the stability of responding in that set (i.e., perfect responding occurred). That data pattern indicates that WSG responding increased across the sets of Phase C and Phase D blocks.

It appears that the increase in WSG responding was due to increased TSG training rather than just increased exposure to the attempt cards. For instance, in the last three Phase B blocks (baseline) prior to the start of TSG training in the Phase C blocks, WSG responding was stable at a value of 2 correct WSG response. Although WSG responding decreased from the first Phase $\mathrm{C}$ block to the second Phase $\mathrm{C}$ block, there was an upward shift in the level of the data pattern when TSG training was introduced in the first Phase C block. That pattern offers some support that the introduction of TSG training affected an increase in WSG responding.

The level changes in the data pattern at the end of the second, third, and fourth set of Phase C blocks (i.e., blocks of TSG training) offer some additional support that TSG training affected the increase in WSG responding. The downward shifts in the level from the last block of Phase $\mathrm{C}$ to the Phase B block (baseline) that followed were to values that were below the mean value of WSG responding shown in the preceding set of Phase C blocks. Those downward shifts indicate that when TSG was withheld during the Phase B blocks, WSG responding decreased.

Since the downward shifts to the Phase B block following the second and third set of Phase C blocks were not accompanied by upward shifts to the first Phase C block that followed (e.g., as was the case in the data pattern of Participant 3), the result of a 
downward shift is confounded by the period of time that occurred between the last Phase C block (i.e., the end of the session) and the Phase B block (i.e., the start of the next session). That is, the decreases in WSG responding in the Phase B blocks may have been affected by the period of time (one or two days) between sessions rather than just the withdraw of TSG training; however, after a short latency periods of one block, WSG responding increased in the second and third sets of Phase $\mathrm{C}$ blocks suggesting that there was a TSG training affect on WSG responding.

There was an upward shift in level from the Phase B block to the first Phase D block (i.e., the addition of WSG training to TSG training). In addition, there was much less variability in WSG responding in the sets of Phase D blocks compared to the Phase C blocks, which suggests that the addition of WSG training may have served to increase stability. Since WSG responding had been shown to increase across previous blocks of TSG training (i.e., Phase C), it is not clear whether or not the continued increase in WSG responding from the last set of Phase $\mathrm{C}$ blocks to the first set of Phase D blocks was due to increased exposure to TSG training or the addition of WSG training.

In summary, for Participant 4 WSG responding, the data pattern supports a training effect on WSG responding. The pattern also suggests that TSG training affected an increase in WSG responding.

Participant 4 BSG responding. Systematic changes in BSG responding were not seen in the data pattern prior to the addition of WSG training to TSG training (i.e., Phase D blocks). The range between the highest data point and the lowest data point of BSG responding in sets of Phase B blocks (i.e., baseline) prior to the start of TSG training was 
similar to the range between the highest and lowest data point of BSG responding in the sets of Phase C blocks (blocks of TSG training).

The data pattern further indicates that BSG responding did not become more stable across the sets of Phase C blocks (i.e., blocks of TSG training) than it had been across the Phase B blocks prior to the start of TSG training. However, the amount of variability in BSG responding did decrease from the sets of Phase C blocks to the first set of Phase D blocks. The variability again increased from the first to the second set of Phase D blocks, but that was the result of an upward trend across the last three blocks of the set. There also was an increase in the mean value of BSG responding from the sets of Phase C blocks to the sets of Phase D blocks. Since there was not a downward shift in level from the last block of the first set of Phase D block to the Phase B block that followed (i.e., responding did not decrease with training was removed), the pattern offers somewhat limited support for a WSG training effect on BSG responding.

In summary, for Participant 4 BSG responding, the increase in mean BSG responding and the decrease in variability shown in the data pattern supports a training effect on BSG responding. The pattern also suggests that the addition of WSG training to TSG training affected an increase in BSG responding.

Summary of Results in Terms of the Research Questions

The changes in the data patterns described in the previous sections addressed the following research questions. (1) Will increased training on TSG increase the number of demonstrations of WSG and BSG? (2) Will the addition of WSG training to TSG training increase the number of demonstrations of BSG? 
In answer to the first research question, the data patterns of three of the four participants (i.e., Participant 1, Participant 3, and Participant 4) suggested that increased TSG training affected an increase in WSG responding. The data pattern of only one of the four participants (i.e., Participant 1) offered limited support that increased TSG training affected an increase in BSG responding.

In answer the second research question, the data patterns of three of the four participants (i.e., Participant 1, Participant 2, and Participant 3) did not indicate that the addition of WSG training to TSG training affected an increase in BSG responding. The remaining data pattern (i.e., the pattern of Participant 4) suggested that the addition of WSG training to TSG training affected an increase in BSG responding. 


\section{Chapter 5: Discussion}

The present study was an examination of a possible functional relation between (a) TSG and WSG, (b) TSG and BSG, and (c) WSG and BSG. Those relations were addressed by the following research questions. (1) Will increased training on TSG increase the number of demonstrations of WSG and BSG? (2) Will the addition of WSG training to TSG training increase the number of demonstrations of BSG?

The first research question was posed because questions pertaining to a functional relation between TSG and WSG and a functional relation between TSG and BSG remained in the literature. Results of the current study showed that the data patterns of three of the four participants suggested that increased TSG training affected an increase in WSG responding. The data pattern of only one of the four participants offered limited support that increased TSG training affected an increase in BSG responding.

The second research question was posed in order to examine a previous finding in the generalization literature that showed BSG responding to be dependent on WSG training (i.e., Haring, 1985; Haring, Breen, \& Laitinen, 1989). Results of the current study showed that the data pattern of only one of the four participants suggested that the addition of WSG training to TSG training affected an increase in BSG responding. The First Research Question Result

A rather theoretical explanation of the result of the first research question can be found through examining the nature of emergent transitive relations among training stimuli (i.e., Set A, Set B, and Set C). That is, a possible explanation can be found through examining the nature of emergent $\mathrm{BC}$ and $\mathrm{CB}$ relations (i.e., transitive relations 
among training stimuli) following training on BA and CA relations (i.e., the match-tosample initial training that was used).

Demonstrations of BC and $\mathrm{CB}$ are direct tests for BA and CA relations (Sidman \& Tailby, 1982). For instance, in order for the relation BC to be responded to without direct training on the relation, both BA and CA relations must be available in the repertoire. In this case, responding to $\mathrm{BC}$ is a result of the relation both $\mathrm{B}$ and $\mathrm{C}$ each have with the intermediary stimulus A. Thus, if TSG were high after initial training (i.e., if the transitive relation were emergent, not trained for), then the application of training on $\mathrm{BC}$ and $\mathrm{CB}$ (transitive relations) might continue to strengthen BA and CA relations. Since TSG responding was high across the participants after initial training (see Tables 1, 2, 3, and 4), the application of training on BC and CB (i.e., TSG training) may have served to strengthen the BA and CA initial training relations.

It is possible that strengthening BA and CA relations would occasion a situation in which the sameness among stimuli would gain stronger control over responding. For example, stimulus features that are the same among Set B stimuli and other possible sample stimuli (e.g., a group of dark and light circles) could gain stronger control over the stimulus features that are the same among Set A stimuli and other possible comparison stimuli (e.g., the denominator of the correct comparison represents the total number of circles in the sample stimulus).

It then would follow that WSG responding should increase since stimuli involved in the WSG probes (i.e., WSG attempt cards) shared essential features with stimuli in Set A, Set B, and Set C. Hence, as the initial training discriminations BA and CA strengthen 
by way of training on BC and CB relations, the number of correct responses to the WSG discriminations should increase.

Increases in number of correct responses to the BSG discriminations could be explained in the same way. Since stimuli involved in BSG probes shared fewer essential features with the initial training sets (i.e., they were not considered to be part of larger sets that include the training sets), the result would be expected to be less pronounced. As shown in the data, an effect of increased training on BC and C B relations (i.e., TSG training) was less pronounced for BSG responding compared to WSG responding, in that the data pattern of only one participant weakly suggested that TSG training affected an increase in BSG responding.

\section{The Second Research Question Result}

BSG responding has been shown to be a function of WSG training (e.g., Haring, 1985). Theoretically, support for WSG training affecting an increase in BSG responding follows directly from Engelmann and Carnine (1982) in that the addition of WSG training to the initial training sets (i.e., multiple set training) should serve to include a wider range of stimuli that could be present during BSG probes. For instance, after training on multiple sets of stimuli (e.g., training on the initial training sets and the WSG sets), a wider range of stimuli may contain stimulus features that indicate the limits of a certain set. That is, after multiple set training, the limits of stimulus features that may be correctly associated with the availability of reinforcement following a certain response may gain stronger control over responding.

In the current study, sample stimuli involved in WSG attempt cards (Set Y and Set Z) were arranged so that they were part of larger sets that included the sample stimuli 
involved in initial training (i.e., Set B and Set C). Thus, the addition of WSG training (i.e., training on YX and ZX discriminations) to TSG training was the application of training to more sets of stimuli that shared sample stimulus features with sets used in initial training. Therefore, it is likely that the addition of WSG training occasioned a situation in which a larger number of sample stimulus features (i.e., the combination of sample stimuli used on WSG attempt cards and initial training attempt cards) gained control over selections of a larger number of comparison stimulus features (i.e., the combination of comparison stimuli used on WSG attempt cards and initial training attempt cards).

It is likely that during WSG training the features of the sample stimuli on the WSG attempt cards gained stronger control over the selection of comparison stimulus features of the WSG attempt cards. Since the comparison stimuli of the WSG attempt cards were the same as the comparison stimuli of the BSG attempt cards, it follows that with increased WSG training the features of the sample stimuli on WSG attempt cards would gain stronger control over selections of comparison stimuli on the BSG attempt cards. Hence, if there were enough overlap among the features of the sample stimuli on the BSG attempt cards and the features of the sample stimuli on WSG attempt cards (i.e., sample stimuli that previously had gained control over comparison stimuli on BSG attempt cards), an increase in BSG responding would be expected. As shown in the data, that expected increase in BSG responding was suggested in the data pattern of only one of the four participants (i.e., Participant 4).

The data patterns of the other participants (Participants 1, 2, and 3) may not have shown an increase in BSG responding following WSG training as a result of the presence 
of TSG training. That is, if BSG responding had started to increase as a result of TSG training, then an increase in responding when WSG was added to TSG training (i.e., Phase D) could not be attributed to the presence of WSG training along (e.g., the data pattern of Participant 1). Thus, WSG training may have affected an increase in BSG responding, but the interpretation of the increase as due to WSG training was confounded by the presence of TSG training.

\section{Relations Among Types of Generalization}

Increased training on TSG was shown to affect an increase in WSG responding for three of the four participants. That suggests that training on certain relations among training stimuli (i.e., transitive relations) occasioned an extension of responding to a larger number of stimuli that shared essential stimulus features (i.e., stimuli on the WSG attempt cards).

The data pattern of only one participant offered limited support that increased training on TSG affected an increase in BSG responding. That suggests that training on certain relations among training stimuli (i.e., transitive relations) did not often occasion an extension of responding to a larger number of stimuli that did not share all essential stimulus features (i.e., stimuli on the BSG attempt cards).

The data pattern of one of the four participants suggested that the addition of WSG training to TSG training affected an increase in BSG responding. That suggests that training on multiple sets of stimuli did not often occasion an extension of responding to a larger number of stimuli that did not share all essential stimulus features (i.e., stimuli on the BSG attempt cards). 
The design of the present study allowed the individual data patterns of WSG and BSG responding to be examined. Analyses of individual data patterns reveals changes and patterns in data that larger group comparisons could mask. Due to the design that was used, the way in which generalization training affected individual participant responding could be addressed; however, there were limitations to what could be seen in the data patterns in terms of the affect that certain types of generalization training had on the demonstration of other types of generalization (e.g., the affect of WSG training on BSG responding). Increasing the length of Phase B (baseline), Phase C (TSG training) and Phase D (WSG and TSG training) would allow for clearer stability patterns and, as a result, decrease some limitations on what changes could be seen in data.

\section{Additional Observations}

Available reinforcement. Although results showed that TSG training did not have much of an affect on BSG responding, there may have been a relation between increased training and BSG responding. TSG training might have occasioned contact with contingencies that served to point out which type of sample stimuli implied the availability of reinforcement following correct selections of comparison stimuli in the form of either (1) the researcher verifying whether or not the comparison selection was correct or (2) avoiding being asked to continue selecting comparison stimuli until the correct comparison was selected (i.e., moving faster through the presentations of attempt cards).

For instance, BSG attempt cards were the only attempt cards not to contain sample stimuli that were fraction pictures of circles. As training was applied to TSG attempt cards (i.e., cards that contained sample stimuli that were fraction pictures of 
circles), the sample stimuli of WSG attempt cards (i.e., other types of attempt cards that contained sample stimuli that were fraction pictures of circles) may have been more likely than the sample stimuli of the BSG attempt cards to become associated with the availability of reinforcement following a correct comparison selection. Similarly, since training was never applied to attempt cards with sample stimuli in the form of fraction pictures of partitioned rectangles (like the sample stimuli of BSG attempt cards), the BSG attempt cards may have become associated with the availability of reinforcement in the form of moving quickly through each presentation of a BSG attempt card following the selection of any (i.e., correct or incorrect) comparison stimulus.

Use of rules. Two of the participants, without prompting, explained how they selected which comparison to pick based on the sample that was presented. The explanations given by those two participants were descriptions of "rules” that could be used to correctly select the correct comparison. For example, one of the participants said, "the number of dark circles is this number" while pointing to the numerator of the correct comparison stimulus.

It was clear that each participant used a rule at different points to make comparison selections, even if the use of that rule did not consistently return correct answers. For example, without prompting from the researcher, each participant at different points counted objects in the sample stimuli of all types of attempt cards before selecting a comparison. Based on such observations, it seems as though each participant had in his or her repertoire a rule or several rules that could be used to increase the likelihood of a correct response. 


\section{Further Research}

The apparent use of rules, in addition to the possible effect of training that served to point out contingencies that enabled participants to move faster through the presentations of attempt cards, suggests that the lack of an increase in BSG responding with increased training does not necessarily imply that generalization (i.e., an extension of responding based on past experience with related stimuli) did not occur. Rather, the topology of the generalization may have been different from what was expected (i.e., BSG responding did not increase), possibly as a consequence of training serving to point out reinforcing contingencies that did not require BSG responding to increase.

Based on the possible effect of training serving to point out reinforcing contingencies that did not require BSG responding to increase, further work is needed to better described the relation between TSG training and BSG responding. For instance, including a measure for evaluating participants' use of “rules” for correctly selecting comparison stimuli during TSG training could be included in a future study. A measure of the use of rules could offer information on when certain types of associations between the stimulus features of sample and comparison stimuli and the availability of reinforcement occur. For instance, measuring behaviors such as counting objects in the sample stimuli of attempt cards could be used to identify rules (e.g., the number of dark circles is the numerator) used for the selection of comparison stimuli. However, an assessment of participants' counting skills would need to be done prior using behaviors such as counting objects in the sample stimulus as a measure of the use of rules. 


\section{Contribution}

The present study points out difficulties in examining generalization in tasks that participants may not find pleasurable to perform (i.e., the performance of the task alone is not reinforcing). For example, it is likely that being finished with the fraction task used in the present study was more reinforcing than matching the comparison stimuli to the sample stimuli. As a result, it is possible that training affected generalization (i.e., an extension of responding based on past experience with related stimuli), but not in the manner that was intended. That is, training may have pointed out contingencies that allowed participants to move faster through the presentations of attempt cards by (1) responding correctly to attempt cards that were similar to attempt cards that received training (and hence could not be moved past until a correct comparison selection was made) and (2) responding incorrectly to attempt cards that were not as similar to attempt cards that received training (and hence could be moved past as soon as any comparison selection was made).

From an applied stance, the present study suggests that training (or teaching) a small number of relations among a small set of training stimuli (e.g., the transitive relations) can occasion an extension of responding to a larger number of related stimuli. Thus, the implication of this study for teachers is that teaching many examples from sets of stimuli that represent a wide variety of important stimulus features should lead to more instances of generalization.

From a basic research stance, the present study offers more insight into the relation between transitive stimulus generalization (TSG) and within-sets generalization 
(WSG). In particular, the present study suggests that WSG responding is functionally related to TSG training. 


\section{References}

Adams, G. L., \& Engelmann, S. (1996). Research on direct instruction: 25 years beyond DISTAR. Seattle, WA: Educational Achievement Systems.

Bandura, A. (1969). Principles of behavior modification. New York: Holt, Rinehart, \& Winston.

Bandura, A. (1977). Social learning theory. Englewood Cliffs, NJ: Prentice-Hall.

Barnes, D. (1994). Stimulus equivalence and relational frame theory. The Psychological Record, 44, 91-124.

Bruner, J. E. (1960). The process of education. New York: Vintage.

Bruner, J. E. (1961). The act of discovery. Harvard Educational Review, 31, 21-32.

Eikeseth, S., \& Smith, T. (1992). The development of functional and equivalence classes in high-functioning autistic children: The role of naming. Journal of the Experimental Analysis of Behavior, 58, 123-133.

Engelmann, S., Becker, W. C., Carnine, D., \& Gersten, R. (1988). The direct instruction follow through model: Design and outcomes. Education and Treatment of Children, 11, 303-317.

Engelmann, S., \& Carnine, D. (1982). Theory of instruction. New York: Irvington.

Fields, L. \& Hobbie-Reeve, S. A. (1999). Effects of training directionality and class size on equivalence class formation by adults. The Psychological Record, 49, 703-724.

Garcia, E., Baer, D. M., \& Firestone, I. (1971). The development of generalized imitation within topographically determined boundaries. Journal of Applied Behavior Analysis, 4, 101-112. 
Good, T. L., \& Brophy, J. (1995). Contemporary educational psychology. White Plains, NY: Longman.

Haring, T. G. (1985). Teaching between-class generalization of toy play behavior to handicapped children. Journal of Applied Behavior Analysis, 18, 127-139.

Haring, T. G., Breen, C. G., \& Laitinen, R. E. (1989). Stimulus class formation and concept learning: Establishment of within- and between-set generalization and transitive relationships via conditional discrimination procedures. Journal of the Experimental Analysis of Behavior, 52, 13-25.

Holman, J., Goetz, E. M., and Baer, D. M. (1977). The training of creativity as an operant and an examination of its generalization characteristics. In B. C. Etzel, J. M. Le Blanc, and D. M. Baer (Ed.), New developments in behavioral research: Theory, methods, and applications. In honor of Sidney W. Bijou (pp. 441-471). Hillsdale, New Jersey: Lawrence Erlbaum Associates.

Innis, A., Lane, S. D., Miller, E. R., \& Critchfield, T. S. (1998). Stimulus equivalence: Effects of a default-response option on emergence of untrained stimulus relations. Journal of the Experimental Analysis of Behavior, 70, 87-102.

Keller, F. S. \& Schoenfeld, W. N. (1950). Principles of psychology. New York: Appleton-Century-Crofts.

Lane, S. D., \& Critchfield, T. S. (1998). Classification of vowels and consonants by individuals with moderate mental retardation: Development of arbitrary relations via match-to-sample training with compound stimuli. Journal of Applied Behavior Analysis, 31, 21-41. 
Lynch, D. C., \& Cuvo, A. J. (1995). Stimulus equivalence instruction of fraction-decimal relations. Journal of Applied Behavior Analysis, 28, 115-126.

Michael, J. (1982). Distinguishing between discriminative and motivational functions of stimuli. Journal of the Experimental Analysis of Behavior, 37, 149-155.

Parsonson, B. S., \& Baer, D. M. (1978). Training generalized improvisation of tools by preschool children. Journal of Applied Behavior Analysis, 11, 363-380.

Pilgrim, C., \& Galizio, M. (1990). Relations between baseline contingencies and equivalence probe performances. Journal of the Experimental Analysis of Behavior, 54, 213-224.

Saunders, R. R., Drake, K. M., \& Spradlin, J. E. (1999). Equivalence class establishment, expansion, and modification in preschool children. Journal of the Experimental Analysis of Behavior, 71, 195-214.

Saunders, R. R., \& Green, G. (1999). A discrimination analysis of training-structure effects on stimulus equivalence outcomes. Journal of the Experimental Analysis of Behavior, 72, 117-137.

Sidman, M. (1990). Equivalence relations: Where do they come from? In D. E. Blackman \& H. Lejeune (Eds.), Behavior analysis in theory and practice: Contributions and controversies (pp. 93-114). Hillsdale, NJ: Erlbaum.

Sidman, M., Kirk, B., \& Willson-Morris, M. (1985). Six-member stimulus classes generated by conditional-discrimination procedures. Journal of the Experimental Analysis of Behavior, 43, 21-42. 
Sidman, M., \& Tailby, W. (1982). Conditional discrimination vs. matching to sample: An expansion of the testing paradigm. Journal of the Experimental Analysis of Behavior, 37, 5-22.

Silbert, J., Carnine, D., \& Stein, M. (1981). Direct instruction mathematics. Columbus, OH: Bell \& Howell.

Skinner, B. F. (1953). Science and human behavior. New York: Free Press.

Skinner, B. F. (1957). Verbal behavior. Acton, MA: Copley Publishing Group.

Spradlin, J. E., \& Saunders, R. R. (1986). The development of stimulus classes using match-to-sample procedures: Sample classification versus comparison classification. Analysis and Intervention in Development Disabilities, 6, 41-58.

Stokes, T. F., \& Baer, D. M. (1977). An implicit technology of generalization. Journal of Applied Behavior Analysis, 10, 349-367.

Tennyson, R. J. (1973). Effect of negative instances in concept acquisition using a verbal learning task. Tallahassee, FL: Center for CAIR. (ERIC Document Reproduction Service No. ED075990)

Werts, M. G. (1991). Stimulus equivalence established through instructive feedback. Pittsburgh, PA: Allegheny-Singer Research Inst. (ERIC Document Reproduction Service No. ED407751)

White, W. A. T. (1988). A meta-analysis of the effects of direct instruction in special education. Education and Treatment of Children, 11, 364-374.

Williams, D. C., Saunders, K. J., Saunders, R. R., \& Spradlin, J. E. (1995). Unreinforced conditional selection within three-choice conditional discriminations. The Psychological Record, 45, 613-627. 
Zentall, T. R., Galizio, M., \& Critchfield, T. S. (2002). Categorization, concept learning, and behavior analysis: An introduction. Journal of the Experimental Analysis of Behavior, 78, 237-248. 
Sample Part One and Part Two Initial Training Scorecards

Participant Number Condition 1

Phase A ( Part 1 Training )

Date
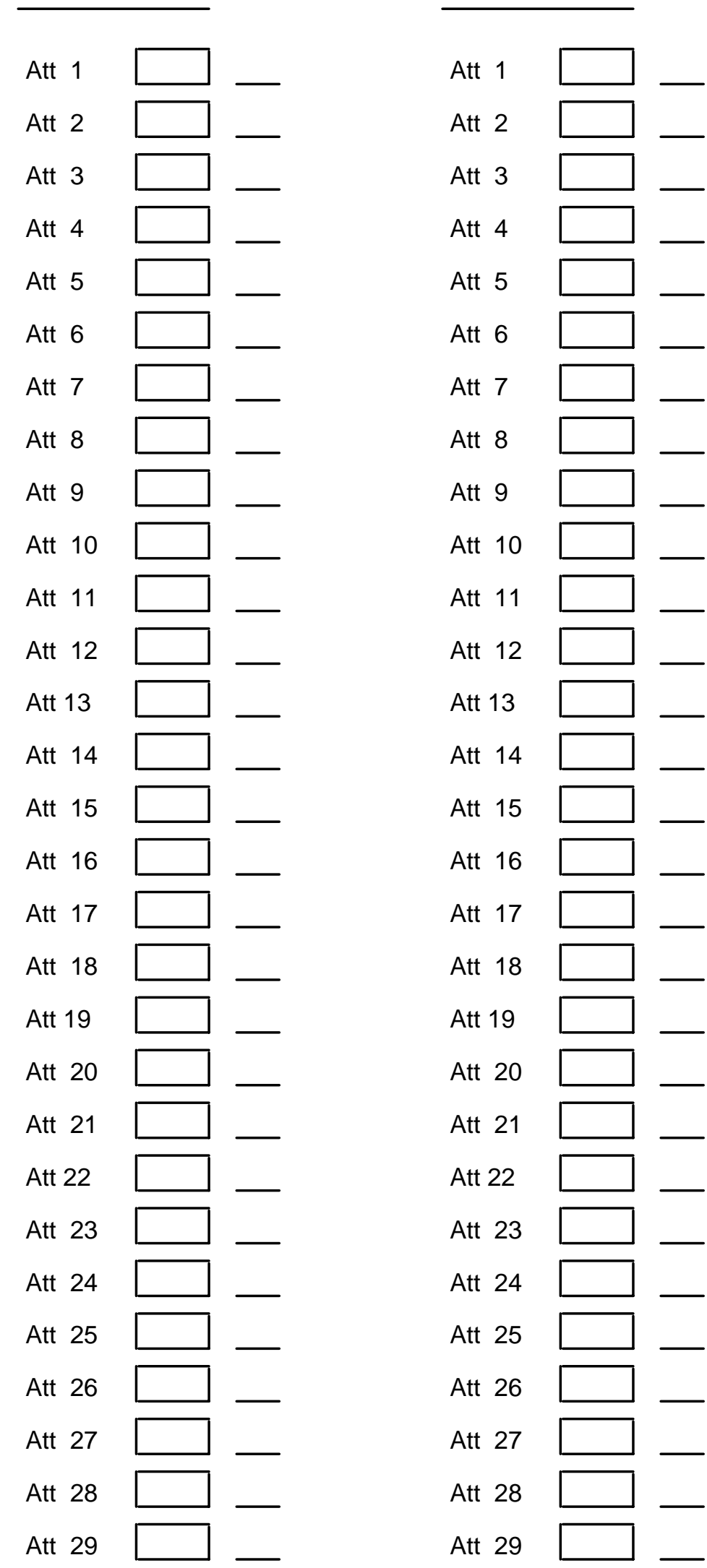

Att 1

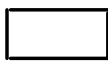

Att 2

Att 3

Att 4

Att 5

Att 6

Att 7

Att 8

Att 9

Att 10

Att 11

Att 12

Att 13

Att 14

Att 15

Att 16

Att 17

Att 18

Att 19

Att 20

Att 21

Att 22

Att 23
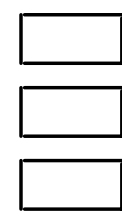

$\longrightarrow$

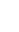
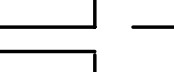


\section{Sample Part One and Part Two Initial Training Scorecards}

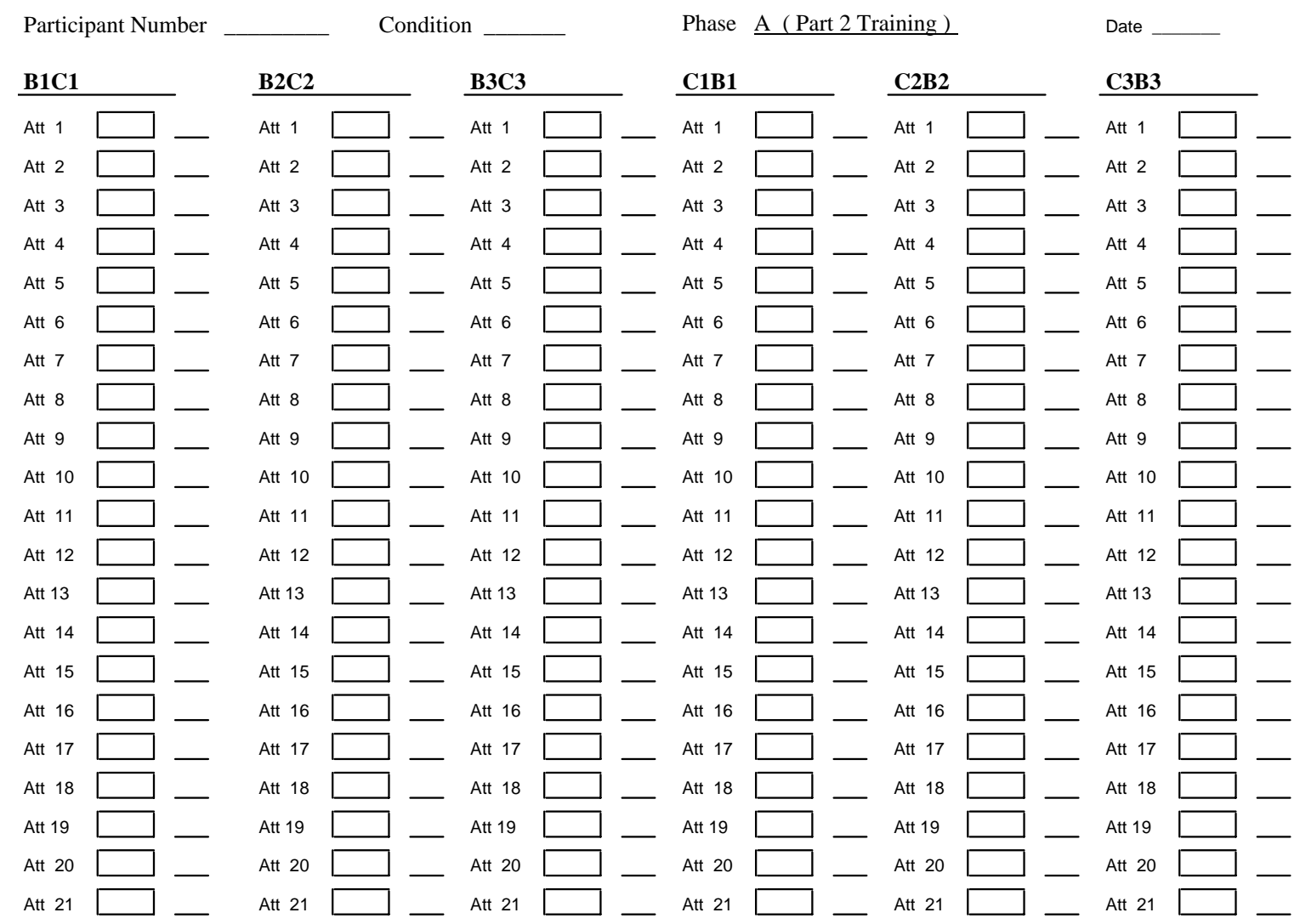


Sample Ordering of Attempt Cards in Two Baseline Blocks

Transitive

Stimulus

Generalization
Within-Sets

Generalization
Between-Sets

Generalization
Training

Discriminations

\begin{tabular}{|c|c|c|c|}
\hline B1C1 & $\mathrm{Y} 2 \mathrm{X} 2$ & F1D1 & B1A1 \\
\hline C2B2 & Y1X1 & E1D1 & C2A2 \\
\hline B3C3 & Z3X3 & E2D2 & B2A2 \\
\hline C3B3 & Z2X2 & E3D3 & C3A3 \\
\hline B2C2 & Y3X3 & F3D3 & C1B1 \\
\hline C1B1 & Z1X1 & F2D2 & B3A3 \\
\hline C3B3 & Y1X1 & F2D2 & $\mathrm{C} 2 \mathrm{~A} 2$ \\
\hline C1B1 & Y3X3 & E2D2 & B3A3 \\
\hline B3C3 & $\mathrm{Y} 2 \mathrm{X} 2$ & F3D3 & B1A1 \\
\hline C2B2 & Z1X1 & F1D1 & C3A3 \\
\hline B1C1 & Z3X3 & E1D1 & B2A2 \\
\hline B2C2 & Z2X2 & E3D3 & C1B1 \\
\hline
\end{tabular}


Sample Baseline Block Scorecard

Participant Number

Condition

Phase

Date

$\stackrel{\text { TSG }}{\square}$

WSG

BSG

Training

TSG

WSG

BSG

Training

Block

TSG

WSG

BSG

Training

TSG

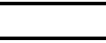

BSG

Training

TSG

WSG

WSG

BSG

Training

TSG

WSG

BSG

Training

$+$

TSG

WSG

BSG

Training

TSG

WSG

BSG

Training

Block

TSG

WSG

BSG

Training

TSG

WSG

BSG

Training

TSG

WSG

BSG

Training

TSG

TSG

WSG

BSG

Training

TSG

WSG

BSG

Training

-

$$
\text { WSG }
$$

TSG

$\square$

BSG

Training

Block

\begin{tabular}{ll} 
TSG \\
\hline TSG \\
\hline TSG \\
\hline TSG \\
\hline \\
\hline
\end{tabular}

BSG

Training

BSG

Training

BSG

Training

BSG

Training 